\title{
2010
}

\section{Y-12 National Security Complex Annual Illness and Injury Surveillance Report}

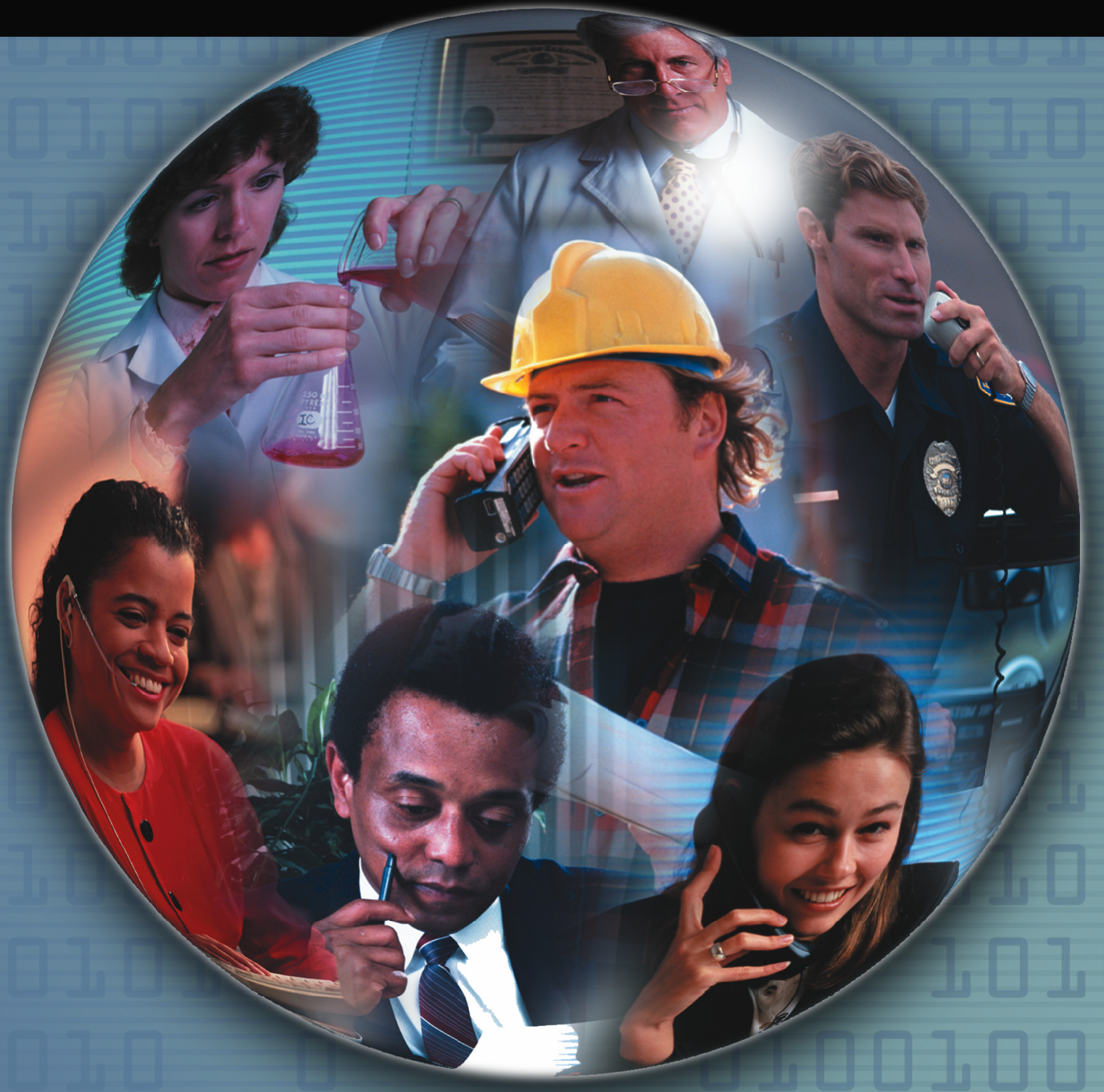




\section{Y-12 National Security Complex 2010 Illness and Injury Surveillance Report}

Questions or comments about this report or the Illness and Injury Surveillance Program (IISP) may be directed to:

E-mail:

Dr. Cliff Strader at cliff.strader@hq.doe.gov or Dr. Bonnie Richter at bonnie.richter@hq.doe.gov

or direct letters to:

Mail Stop HS-13 / GTN Building

U.S. Department of Energy

1000 Independence Avenue, S.W.

Washington, DC 20585-0270

Additional information about the Department of Energy's Office of Illness and Injury Prevention Programs, the IISP, and annual reports for DOE sites participating in this program can be found at:

http://www.hss.energy.gov/healthsafety/WSHP/epi/surv/

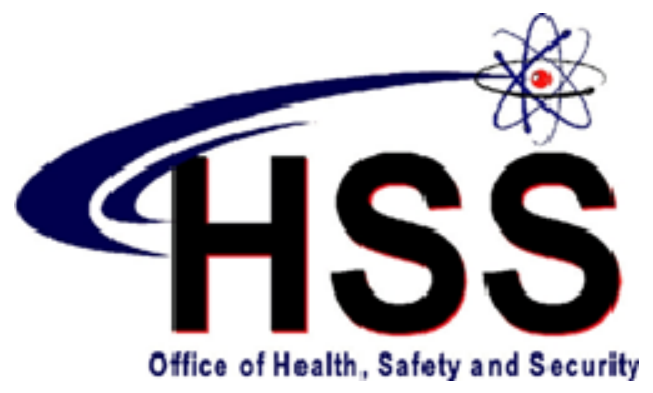

ACKNOWLEDGEMENT

LifeART images copyright 2000 Lippincott Williams \& Wilkins. All rights reserved.

This document was produced under contract number DE-AC05-06OR23100 between the

U.S. Department of Energy and Oak Ridge Associated Universities. 


\section{Y-12 National Security Complex \\ 2010 Illness and Injury Surveillance Report}

At A Glance

NOTE: In previous years, the number of days absent or lost and the age of the work force were reported as averages. Beginning in 2010, they are being reported as medians. A median is the value in a population that half the values exceed and half fall short of it. The median is less affected than the average when the distribution contains a few very large or very small values and provides a more representative summary measure.

The Y-12 work force consisted of 5,017 employees in 2010. The size of the work force has increased less than one percent from 2006 to 2010. Women made up about 27 percent of all workers, which is consistent with other DOE sites participating in the Illness and Injury Surveillance Program (IISP).

There were 935 absences reported in 2010, a 14 percent increase from 2006. The increase in absences was not due to any particular occupational group or diagnosis category. The median length of absences for women was 20 days and for men was 26 days. The median length of absences for women increased 3 days and for men decreased 1 day compared with 2006. Over the 5-year period, the median length of absences was lowest in 2008 and 2009 for both men and women. In 2010, the median was at the highest point (20 days for women) or near the highest point (26 days for men) for Y-12 workers.

Women lost 13,602 calendar days due to injury and illness, a 41 percent increase from 2006 and the highest number of lost days for the 5-year period. Men lost 25,235 calendar days due to injury and illness, representing a 9 percent increase from 2006. The number of lost days for men in 2010 was typical of the days lost for a majority of the years since 2006. 
Among women, the top three diagnoses were musculoskeletal and respiratory conditions, and unspecified symptoms. Unspecified symptoms consisted of mainly digestive symptoms and other symptoms, of which one-third were abdominal pain or swelling. The most common diagnostic categories among men were musculoskeletal conditions, injuries, and respiratory conditions. Musculoskeletal and respiratory conditions accounted for more than 40 percent of diagnoses among men and women at Y-12 in 2010 and are typically among the 3 most commonly reported at DOE sites participating in the IISP.

Age-adjusted rates for all illness and injury diagnoses combined increased for both men and women at Y-12 in 2010. An increase in the rate of musculoskeletal conditions appears to be the main cause of this increase. An increase in the number of back disorders is responsible for the increase in the rate of musculoskeletal conditions in both men and women.

Line Operators had the highest absence rate among men and women in 2010. Security and Fire workers had the lowest absence rates for men and women, which is not typically seen among DOE sites participating in the IISP.

Seventeen possible diagnoses for sentinel health events were reported in 2010. Eight diagnoses involved carpal tunnel syndrome, reported by 5 women and 3 men, all of whom were 40 years or older and from various occupational groups. Carpal tunnel syndrome is among the more commonly observed sentinel health events at IISP sites.

There were 64 OSHA-recordable events among Y-12 workers in 2010, approximately 50 percent more events than were reported in 2009 . The rate of OSHA events was the same for both years (1 event per 100 workers). The rate of OSHA events has been stable at Y-12 since 2006, mainly because of low numbers of OSHA events each year in relation to the size of the work force.

Injuries accounted for 69 percent of the OSHA diagnoses reported by men and 94 percent of the diagnoses among women. The most common types of injuries among men and women were sprains and strains, bruises, and open wounds. 
Crafts workers had the highest rate of OSHA events among both women (20 events per 100 workers) and men (4 events per 100 workers). No OSHA events were reported by workers in the Administrative Support, Technical Support, Security and Fire, and Line Operators occupational categories. This is not typical for Security and Fire workers among DOE sites participating in IISP; their rate of OSHA events has been among the three highest rates among DOE sites participating in the IISP since 2006. The remaining job categories that reported no OSHA events at Y-12 have typically had low rates of OSHA events at other DOE IISP sites since 2006.

For women at Y-12, the number of OSHA events increased from 10 events in 2009 to 16 events in 2010. The number of lost or restricted days for these events was more than 5 times greater in 2010 (265 days) than the number of days in 2009 (49 days). The high number of lost and restricted days in 2010 was due, in part, to two long absences (a total of 243 days lost or restricted). Both events were the result of a fall. The number of OSHA events for men increased from 32 events in 2009 to 48 events in 2010. The number of days lost or restricted was 2.5 times greater in 2010 (1,120 days) than in 2009 (448 days). Five events reported by men accounted for 756 lost and restricted workdays. Three events were due to falls and 2 events were due to overexertion and strenuous movements. 
The Y-12 National Security Complex

Work Force - 2010

The Work Force by Gender and Age ............ 1

The Work Force by Gender and Job

Category 1

\section{Number and Length of Absences}

Absence Rate by Gender and Age 2

Number of Days Absent by

Gender and Age

Absence Rate by Job Category

and Gender .. 3

Median Duration of Absence by

Job Category and Gender. .. 3

\section{Diagnostic Categories}

Number of Diagnoses and Lost Calendar Days by Diagnostic Category

(Categorized by ICD-9-CM) and Gender

Common Diagnoses Among Female

Workers in 2010 .

Common Diagnoses Among Male

Workers in 2010 . 6

Number of Most Frequently Reported

Diagnoses by Job Category and Gender 7

\section{Rates of Disease Occurrence}

Rates for All Illnesses and Injuries Combined by Job Category, Gender, and Age

Rates for Selected Diagnostic Categories by Job Category, Gender, and Age . .8

\section{Time Trends}

Age-Adjusted Rates for All Diagnoses Combined Among Women and Men from 2006 to 2010
Age-Adjusted Rates for Selected Diagnostic Categories Among Women and Men from 2006 to 2010

Age-Adjusted Rates for All Diagnoses

Combined Among Women and Men by Job

Category from 2006 to $2010 \ldots \ldots \ldots \ldots \ldots \ldots \ldots \ldots . . . .12$

\section{Sentinel Health Events for Occupations (SHEOs)}

Characteristics of SHEOs by Gender 13

SHEO Diagnoses by Gender 13

Occupational Safety and Health Administration (OSHA)-Recordable Events

OSHA-Recordable Events by Gender and Age. 14

OSHA-Recordable Events by Job

Category and Gender 14

\section{Diagnostic and Accident Categories for OSHA-Recordable Events}

OSHA-Recordable Diagnoses by

Diagnostic Category and Gender

OSHA-Recordable Accidents by Type

and Gender

Rates of OSHA-Recordable Events

OSHA-Recordable Rates by Age and Job

Categories Among Women, All Diagnoses

Combined

OSHA-Recordable Rates by Age and Job

Categories Among Men, All Diagnoses

Combined

Time Trends for OSHA-Recordable Events

Age-Adjusted Rates for All OSHA-Recordable Diagnoses Combined Among Women and Men by Job Category from 2006 to 2010 .

\section{Appendices}

Appendices A-W .20 
The Y-12 National Security Complex Work Force - 2010

Figure 1. The Work Force by Gender and Age

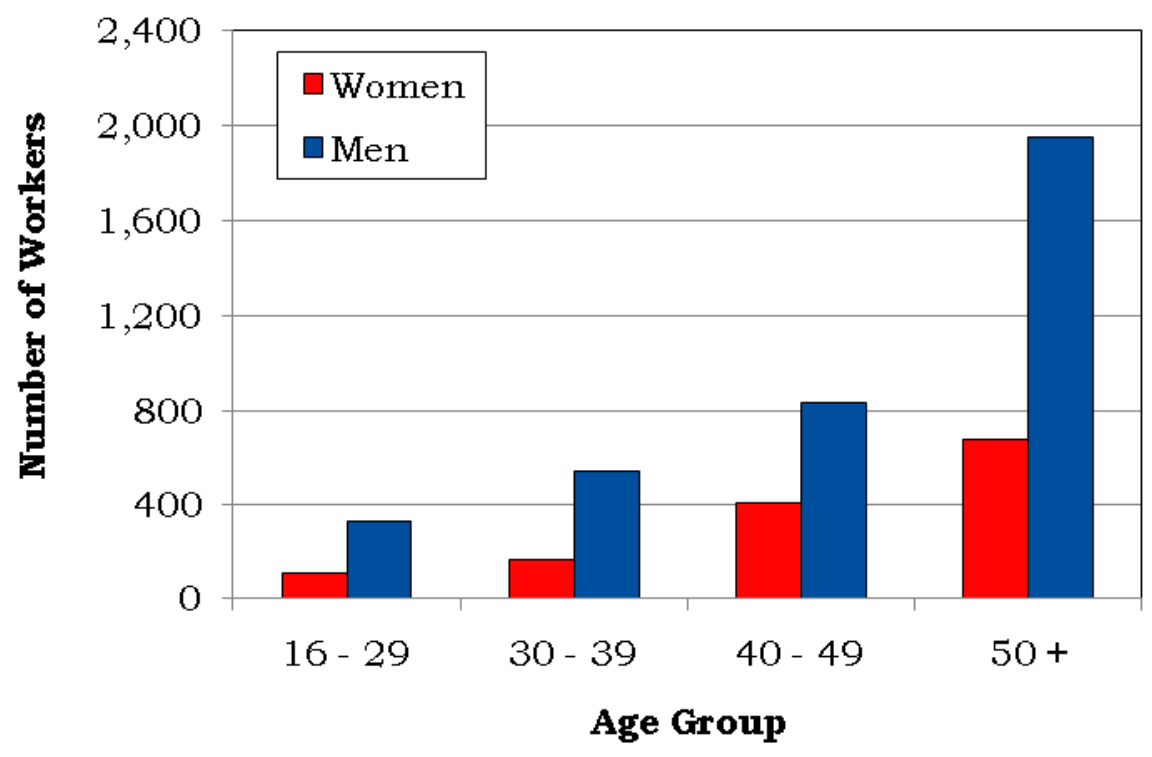

Figure 2. The Work Force by Gender and Job Category

\begin{tabular}{|c|c|c|}
\hline Job Category & Women & Men \\
\hline \multirow{2}{*}{ Professional } & 489 & 1,641 \\
\hline & $36 \%$ & $45 \%$ \\
\hline \multirow{2}{*}{ Administrative Support } & 545 & 351 \\
\hline & $40 \%$ & $9 \%$ \\
\hline \multirow{2}{*}{ Technical Support } & 97 & 143 \\
\hline & $7 \%$ & $4 \%$ \\
\hline \multirow{2}{*}{ Service } & 89 & 375 \\
\hline & $7 \%$ & $10 \%$ \\
\hline \multirow{2}{*}{ Security and Fire } & 47 & 132 \\
\hline & $4 \%$ & $4 \%$ \\
\hline \multirow{2}{*}{ Crafts } & 44 & 829 \\
\hline & $3 \%$ & $23 \%$ \\
\hline \multirow{2}{*}{ Line Operators } & 47 & 188 \\
\hline & $3 \%$ & $5 \%$ \\
\hline \multirow{2}{*}{ Total } & 1,358 & 3,659 \\
\hline & $100 \%$ & $100 \%$ \\
\hline
\end{tabular}




\section{Number and Length of Absences}

Figure 3. Absence Rate by Gender and Age

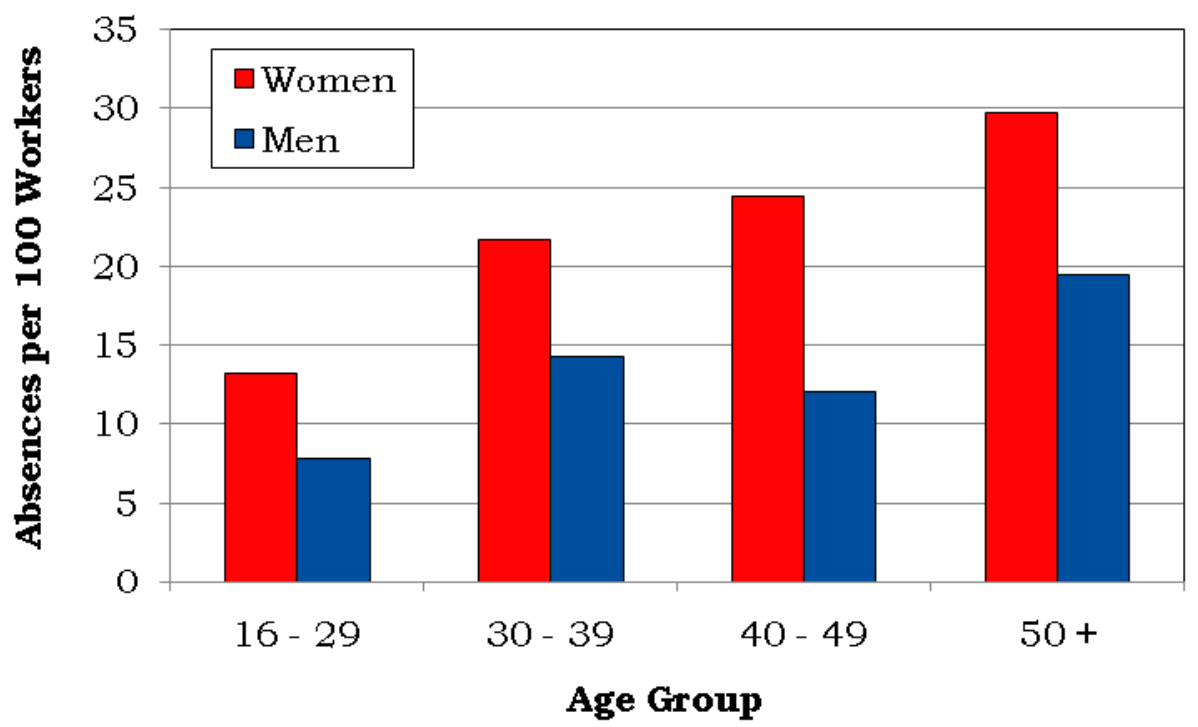

Figure 4. Number of Days Absent by Gender and Age

\begin{tabular}{|c|c|c|c|c|}
\hline \multirow{2}{*}{ Gender } & \multirow{2}{*}{ Age } & \multirow{2}{*}{$\begin{array}{l}\text { Number of } \\
\text { Absences }\end{array}$} & \multicolumn{2}{|c|}{ Number of Days Absent } \\
\hline & & & Total & Median \\
\hline \multirow{5}{*}{ Women* } & $16-29$ & 14 & 526 & 25 \\
\hline & $30-39$ & 36 & 1,145 & 19 \\
\hline & $40-49$ & 100 & 3,307 & 19 \\
\hline & $50+$ & 201 & 8,624 & 21 \\
\hline & Total & 351 & 13,602 & 20 \\
\hline \multirow{5}{*}{ Men } & $16-29$ & 26 & 1,314 & 35 \\
\hline & $30-39$ & 78 & 2,900 & 25 \\
\hline & $40-49$ & 100 & 3,357 & 21 \\
\hline & $50+$ & 380 & 17,664 & 27 \\
\hline & Total & 584 & 25,235 & 26 \\
\hline
\end{tabular}

*Normal pregnancies were excluded from absences for women. 
Figure 5. Absence Rate by Job Category and Gender
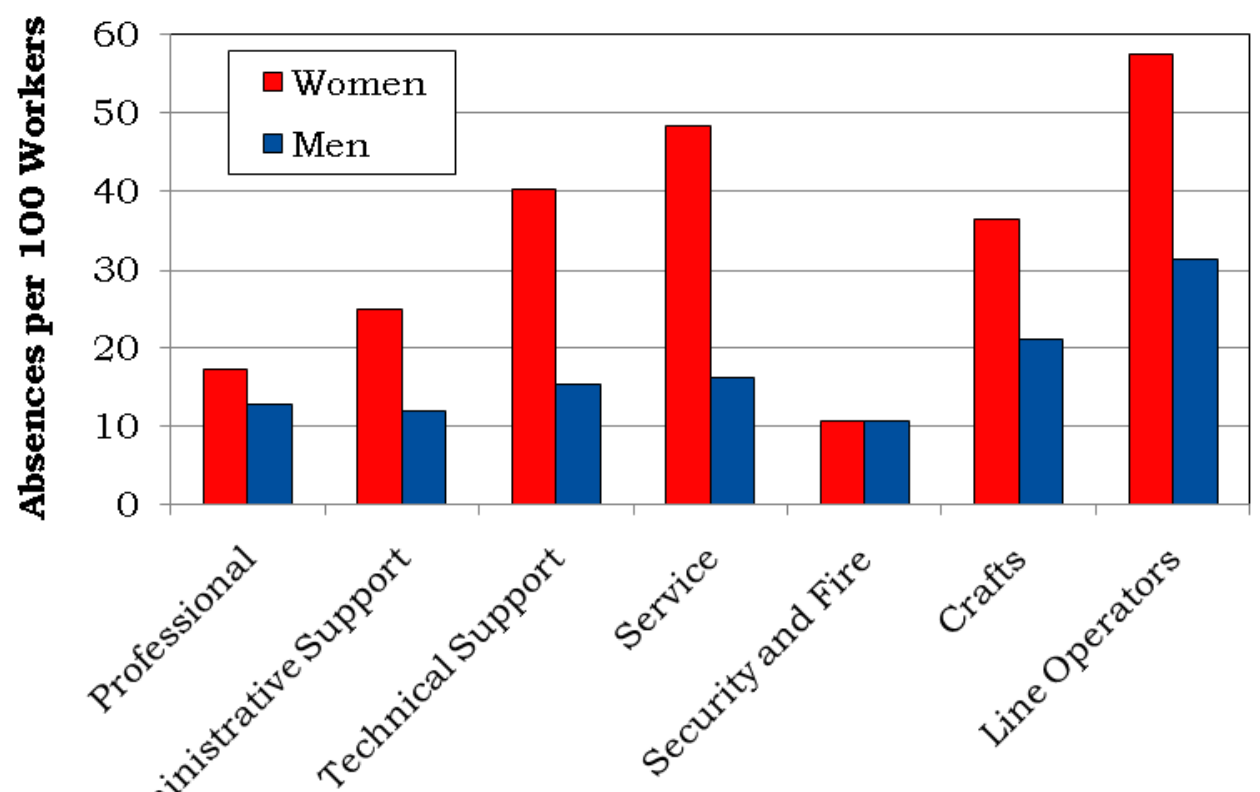

Job Category

Figure 6. Median Duration of Absence by Job Category and Gender

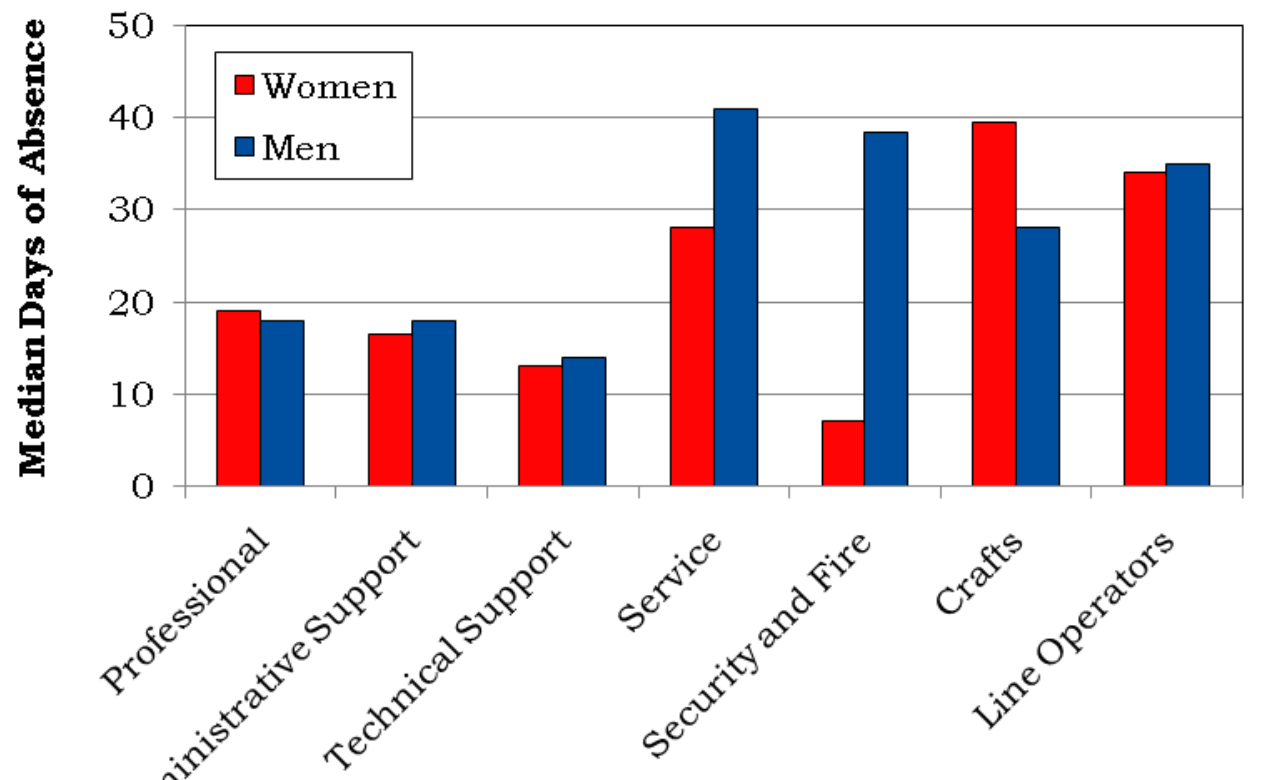

Job Category 


\section{Diagnostic Categories}

Figure 7. Number of Diagnoses and Lost Calendar Days by Diagnostic Category (Categorized by ICD-9-CM) and Gender

\begin{tabular}{|c|c|c|c|c|}
\hline \multirow[b]{2}{*}{ Diagnostic Category } & \multicolumn{2}{|c|}{ Women } & \multicolumn{2}{|c|}{ Men } \\
\hline & $\begin{array}{l}\text { Number of } \\
\text { Diagnoses }\end{array}$ & $\begin{array}{c}\text { Number } \\
\text { of Lost } \\
\text { Calendar } \\
\text { Days }\end{array}$ & $\begin{array}{l}\text { Number of } \\
\text { Diagnoses }\end{array}$ & $\begin{array}{c}\text { Number } \\
\text { of Lost } \\
\text { Calendar } \\
\text { Days }\end{array}$ \\
\hline Benign Growths & 7 & 250 & 10 & 116 \\
\hline Blood & 2 & 215 & 1 & 186 \\
\hline Cancer & 8 & 610 & 22 & 1,394 \\
\hline Digestive & 50 & 1,224 & 77 & 2,149 \\
\hline Endocrine / Me tabolic & 10 & 284 & 21 & 593 \\
\hline Existing Birth Condition & 3 & 97 & 5 & 307 \\
\hline Genitourinary & 47 & 1,448 & 22 & 816 \\
\hline Heart/Circulatory & 23 & 1,363 & 82 & 3,650 \\
\hline Infections/Parasites & 12 & 300 & 12 & 507 \\
\hline Injury & 38 & 1,818 & 118 & 5,355 \\
\hline Miscarriage & 0 & 0 & NA & NA \\
\hline Musculoskeletal & 121 & 5,552 & 248 & 9,759 \\
\hline Nervous System & 37 & 1,401 & 50 & 1,709 \\
\hline Psychological & 12 & 470 & 29 & 1,628 \\
\hline Respiratory & 100 & 1,290 & 108 & 1,334 \\
\hline Skin & 4 & 29 & 24 & 930 \\
\hline Unspecified Symptoms & 53 & 1,463 & 70 & 1,963 \\
\hline
\end{tabular}

Note: Lost calendar days for each absence are counted more than once when multiple diagnoses occur in different diagnostic categories for the same absence. 
Figure 8. Common Diagnoses Among Female Workers in 2010

Intestinal Disorders

Hernias

Gallbladder

Disorders Esophagus/Stomach Appendicitis

All Other

Kidney Disorders

Disorders of the Reproductive Organs

Bladder Disorders

All Other

Sprains and Strains

Open Wounds

Fractures

Dislocations

Bruises

All Other

Rheumatism

Joints

Back

All Other

Upper Respiratory

Pneumonia/Flu Bronchitis/Asthma

All Other

Headache

Fatigue

Dizziness

Digestive Symptoms

Chest Pain

All Other
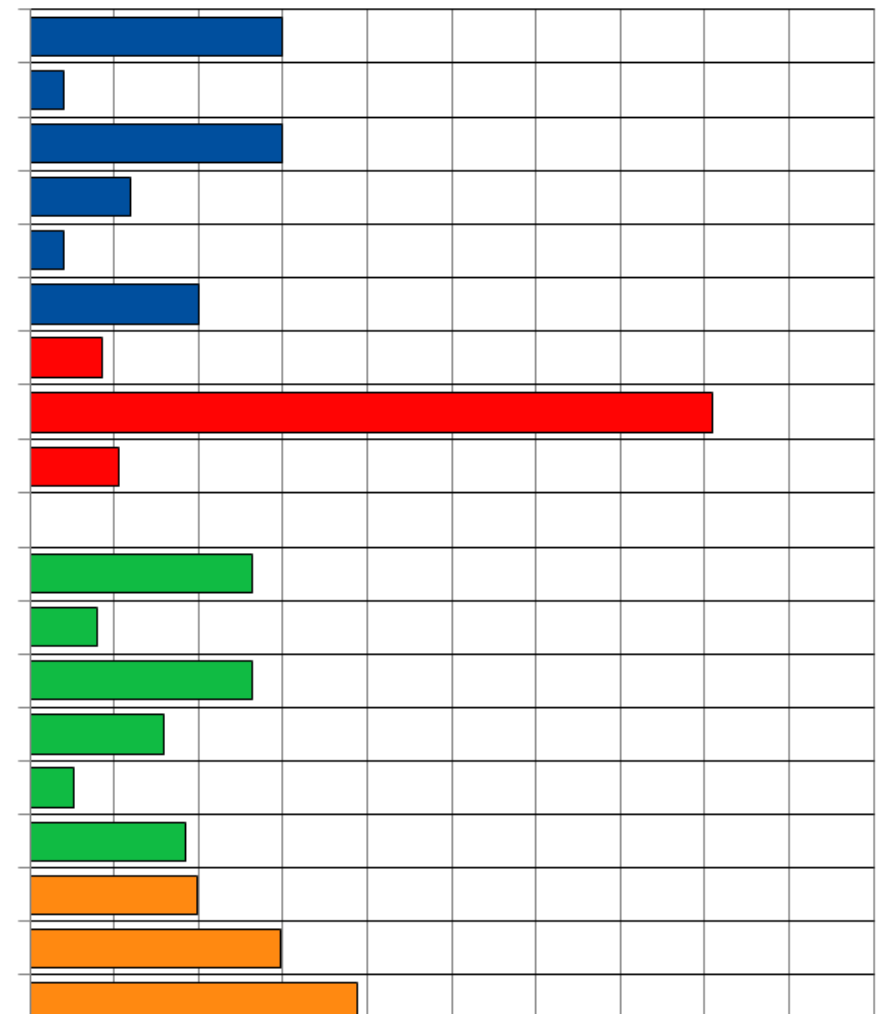

(1)
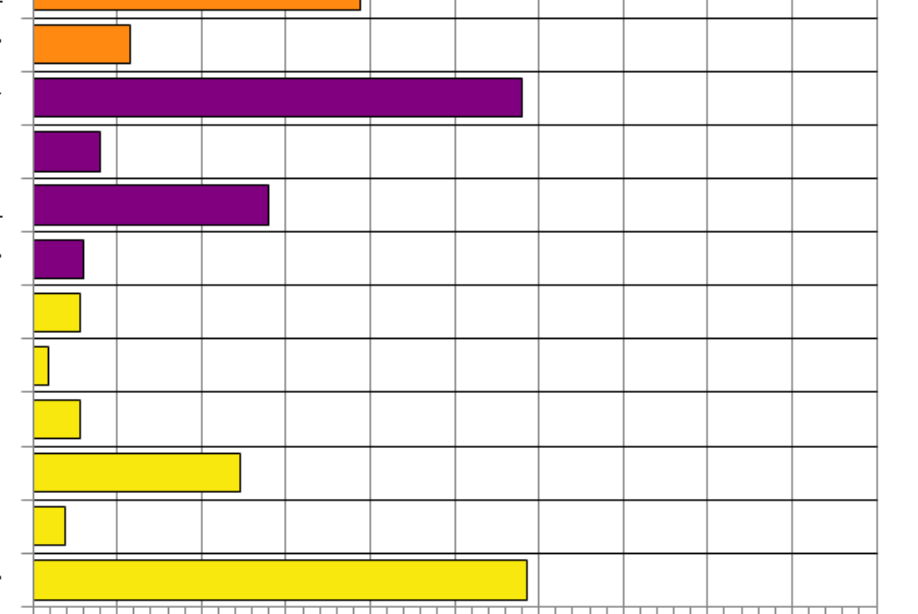

$\begin{array}{lllllllllll}0 & 10 & 20 & 30 & 40 & 50 & 60 & 70 & 80 & 90 & 100\end{array}$

Percent Distribution of Diagnoses Within Diagnostic Category

Dige stive, 50 Diagnoses

Genitourinary, 47 Diagnoses

Injury, 38 Diagnoses
Musculoskeletal, 121 Diagnoses

Respiratory, 100 Diagnoses

Unspecified Symptoms, 53 Diagnoses 


\section{Figure 9. Common Diagnoses Among Male Workers in 2010}

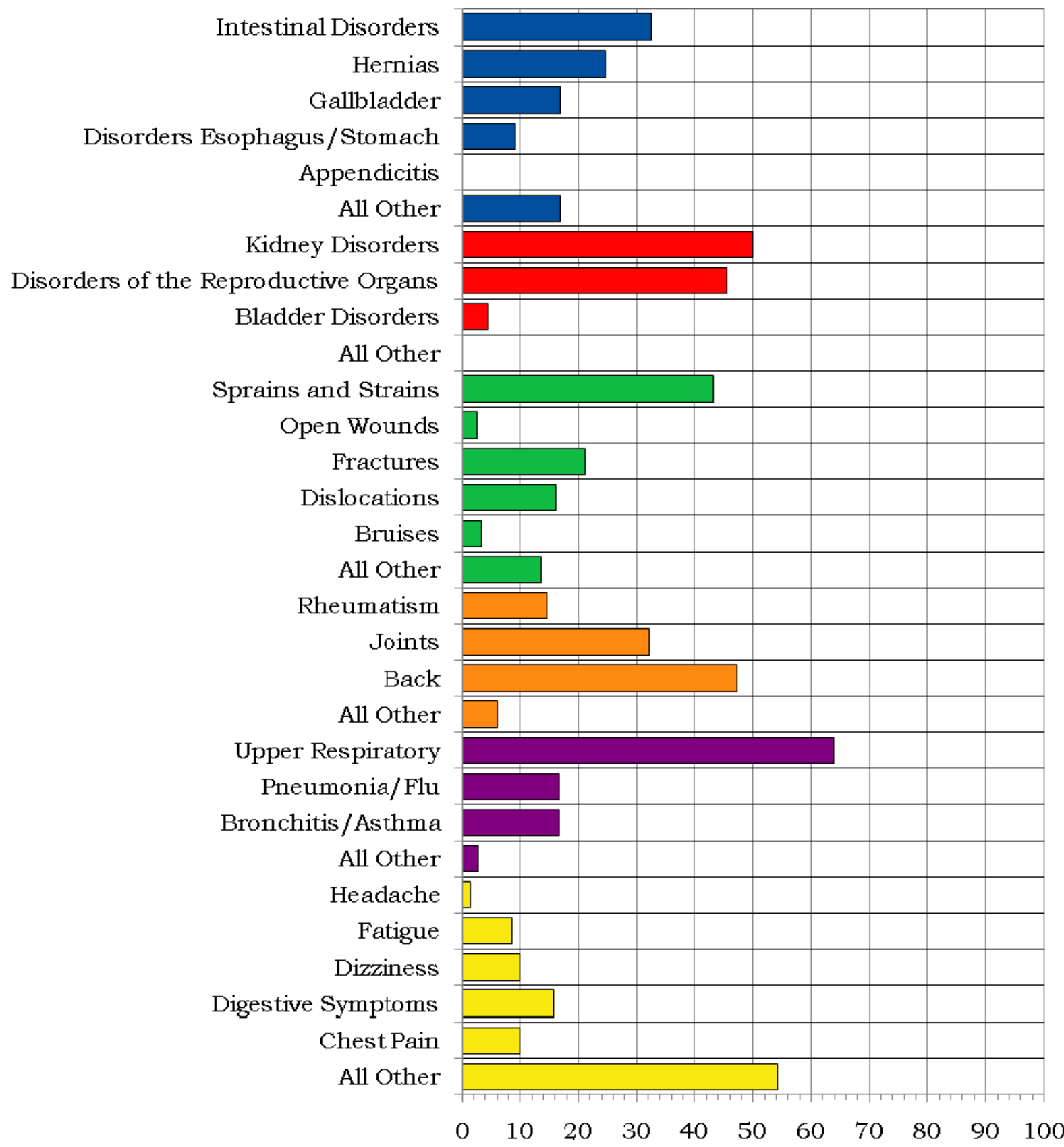

Percent Distribution of Diagnoses Within Diagnostic Category

Digestive, 77 Diagnoses

Genitourinary, 22 Diagnoses

Injury, 118 Diagnoses
Musculoskeletal, 248 Diagnoses

Respiratory, 108 Diagnoses

Unspecified Symptoms, 70 Diagnoses 


\section{Figure 10. Number of Most Frequently Reported Diagnoses by Job Category and Gender}

\begin{tabular}{|c|c|c|c|c|}
\hline Job Category & Men & & Women & \\
\hline \multirow{4}{*}{ Professional } & M usculoskeletal & 94 & M usculoskeletal & 29 \\
\hline & Injury & 47 & Digestive & 13 \\
\hline & Respiratory & 38 & Respiratory & 13 \\
\hline & & & Unspecified Symptoms & 13 \\
\hline \multirow{3}{*}{ Administrative Support } & M usculoskeletal & 12 & Respiratory & 43 \\
\hline & Unspecified Symptoms & 8 & M usculoskeletal & 41 \\
\hline & Digestive & 8 & Digestive & 23 \\
\hline \multirow{4}{*}{ Technical Support } & M usculoskeletal & 8 & Respiratory & 16 \\
\hline & Digestive & 6 & M usculoskeletal & 11 \\
\hline & Injury & 4 & Digestive & 5 \\
\hline & & & Unspecified Symptoms & 5 \\
\hline \multirow{3}{*}{ Service } & M usculoskeletal & 27 & Respiratory & 18 \\
\hline & Respiratory & 11 & M usculoskeletal & 16 \\
\hline & Heart/Circulatory & 10 & Unspecified Symptoms & 11 \\
\hline \multirow{4}{*}{ Security and Fire } & M uscul oskeletal & 10 & Genitourinary & 4 \\
\hline & Heart/Circulatory & 4 & Digestive & 2 \\
\hline & Unspecified Symptoms & 2 & M usculoskeletal & 1 \\
\hline & Genitourinary & 2 & & \\
\hline \multirow{3}{*}{ Crafts } & M usculoskeletal & 64 & M usculoskeletal & 5 \\
\hline & Injury & 41 & Respiratory & 4 \\
\hline & Respiratory & 37 & Nervous System & 3 \\
\hline \multirow{3}{*}{ Line Operators } & M usculoskeletal & 33 & M usculoskeletal & 18 \\
\hline & Injury & 14 & Respiratory & 6 \\
\hline & Respiratory & 13 & Unspecified Symptoms & 4 \\
\hline
\end{tabular}




\section{Rates of Disease Occurrence}

Figure 11. Rates for All Illnesses and Injuries Combined by Job Category, Gender, and Age

\begin{tabular}{|c|c|c|c|c|}
\hline \multirow{2}{*}{$\begin{array}{c}\text { All Illnesses \& } \\
\text { Injuries Combined }\end{array}$} & \multicolumn{4}{|c|}{ Rate per 1,000} \\
\hline & Job Category & Age & Men & Women \\
\hline & \multirow{2}{*}{ Professional } & $<50$ & 123 & 228 \\
\hline & & $50+$ & 260 & 257 \\
\hline & \multirow{2}{*}{ Administrative Support } & $<50$ & 117 & 329 \\
\hline & & $50+$ & 203 & 444 \\
\hline & \multirow{2}{*}{ Technical Support } & $<50$ & 215 & 417 \\
\hline & & $50+$ & 141 & 776 \\
\hline & \multirow{2}{*}{ Service } & $<50$ & 197 & 568 \\
\hline & & $50+$ & 305 & 1,000 \\
\hline & \multirow{2}{*}{ Security and Fire } & $<50$ & 85 & 138 \\
\hline & & $50+$ & 280 & 167 \\
\hline & \multirow{2}{*}{ Crafts } & $<50$ & 258 & 469 \\
\hline & & $50+$ & 393 & 417 \\
\hline & \multirow{2}{*}{ Line Operators } & $<50$ & 430 & 714 \\
\hline & & $50+$ & 635 & 885 \\
\hline
\end{tabular}

Figure 12. Rates for Selected Diagnostic Categories by Job Category, Gender, and Age

\begin{tabular}{|c|c|c|c|c|}
\hline \multirow{2}{*}{ Cancer } & \multicolumn{4}{|c|}{ Rate per 1,000} \\
\hline & Job Category & Age & Men & Women \\
\hline & Profecaiono1 & $<50$ & $\mathbf{0}$ & 0 \\
\hline & 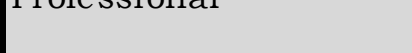 & $50+$ & 9 & 13 \\
\hline & Adminictrative Sunmort & $<50$ & 0 & 4 \\
\hline & H Henthis & $50+$ & 15 & 10 \\
\hline & Tenhnicol Sunnort & $<50$ & $\mathbf{0}$ & 0 \\
\hline & I ecrintedi support & $50+$ & 0 & 0 \\
\hline & & $<50$ & 0 & 0 \\
\hline & pervice & $50+$ & 12 & 0 \\
\hline & Security and Fire & $<50$ & 0 & 0 \\
\hline & & $50+$ & 20 & 0 \\
\hline & Crafts & $<50$ & 5 & 31 \\
\hline & 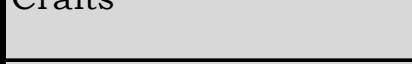 & $50+$ & 12 & 0 \\
\hline & Line Operators & $<50$ & 0 & 0 \\
\hline & & $50+$ & 0 & $\mathbf{0}$ \\
\hline
\end{tabular}


Figure 12. Rates for Selected Diagnostic Categories by Job Category, Gender, and Age (Continued)

\begin{tabular}{|l|l|c|c|c|}
\hline \multirow{2}{*}{ Heart/Circulatory } & \multicolumn{4}{|c|}{ Rate per 1,000 } \\
\cline { 2 - 5 } & \multicolumn{1}{|c|}{ Job Category } & Age & M en & W omen \\
\cline { 2 - 5 } & Profe ssional & $<50$ & $\mathbf{3}$ & $\mathbf{4}$ \\
\cline { 2 - 5 } & $50+$ & $\mathbf{3 5}$ & $\mathbf{4}$ \\
\hline \multirow{2}{*}{ Administrative Support } & $<50$ & $\mathbf{0}$ & $\mathbf{1 2}$ \\
\cline { 2 - 5 } & $50+$ & $\mathbf{1 5}$ & $\mathbf{3 1}$ \\
\cline { 2 - 5 } & \multirow{2}{*}{ Technical Support } & $<50$ & $\mathbf{1 3}$ & $\mathbf{0}$ \\
\cline { 2 - 5 } & $50+$ & $\mathbf{1 6}$ & $\mathbf{4 1}$ \\
\cline { 2 - 5 } & \multirow{2}{*}{ Service } & $<50$ & $\mathbf{0}$ & $\mathbf{0}$ \\
\cline { 2 - 5 } & $50+$ & $\mathbf{6 0}$ & $\mathbf{7 7}$ \\
\cline { 2 - 5 } & \multirow{2}{*}{ Security and Fire } & $<50$ & $\mathbf{2 4}$ & $\mathbf{0}$ \\
\cline { 2 - 5 } & $50+$ & $\mathbf{4 0}$ & $\mathbf{0}$ \\
\cline { 2 - 5 } & \multirow{2}{*}{ Crafts } & $<50$ & $\mathbf{2 3}$ & $\mathbf{3 1}$ \\
\cline { 2 - 5 } & $50+$ & $\mathbf{3 5}$ & $\mathbf{0}$ \\
\cline { 2 - 5 } & \multirow{2}{*}{ Line Operators } & $<50$ & $\mathbf{0}$ & $\mathbf{0}$ \\
\cline { 2 - 5 } & $50+$ & $\mathbf{4 1}$ & $\mathbf{7 7}$ \\
\hline
\end{tabular}

\begin{tabular}{|c|c|c|c|c|}
\hline \multirow{2}{*}{ Respiratory } & \multicolumn{4}{|c|}{ Rate per 1,000} \\
\hline & Job Category & Age & Men & Women \\
\hline & \multirow{2}{*}{ Professional } & $<50$ & 16 & 23 \\
\hline & & $50+$ & 28 & 31 \\
\hline & \multirow{2}{*}{ Administrative Support } & $<50$ & 13 & 63 \\
\hline & & $50+$ & 25 & 92 \\
\hline & \multirow{2}{*}{ Technical Support } & $<50$ & 25 & 104 \\
\hline & & $50+$ & 0 & 224 \\
\hline & \multirow{2}{*}{ Service } & $<50$ & 29 & 243 \\
\hline & & $50+$ & 30 & 173 \\
\hline & \multirow{2}{*}{ Security and Fire } & $<50$ & 0 & 0 \\
\hline & & $50+$ & 0 & 0 \\
\hline & \multirow{2}{*}{ Crafts } & $<50$ & 25 & 125 \\
\hline & & $50+$ & 62 & 0 \\
\hline & \multirow{2}{*}{ Line Operators } & $<50$ & 53 & 48 \\
\hline & & $50+$ & 95 & 192 \\
\hline
\end{tabular}


Figure 12. Rates for Selected Diagnostic Categories by Job Category, Gender, and Age (Continued)

\begin{tabular}{|c|c|c|c|c|}
\hline \multirow{2}{*}{ Injury } & \multicolumn{4}{|c|}{ Rate per 1,000} \\
\hline & Job Category & Age & Men & Women \\
\hline & \multirow{2}{*}{ Professional } & $<50$ & 25 & 27 \\
\hline & & $50+$ & 31 & 18 \\
\hline & \multirow{2}{*}{ Administrative Support } & $<50$ & 6 & 32 \\
\hline & & $50+$ & 10 & 38 \\
\hline & \multirow{2}{*}{ Technical Support } & $<50$ & 38 & 0 \\
\hline & & $50+$ & 16 & 20 \\
\hline & \multirow{2}{*}{ Service } & $<50$ & 24 & 27 \\
\hline & & $50+$ & 18 & 58 \\
\hline & \multirow{2}{*}{ Security and Fire } & $<50$ & 12 & 0 \\
\hline & & $50+$ & 0 & 0 \\
\hline & \multirow{2}{*}{ Crafts } & $<50$ & 56 & 0 \\
\hline & & $50+$ & 44 & 83 \\
\hline & \multirow{2}{*}{ Line Operators } & $<50$ & 44 & 48 \\
\hline & & $50+$ & 122 & 38 \\
\hline
\end{tabular}

Time Trends

Figure 13. Age-Adjusted Rates for All Diagnoses Combined Among Women and Men from 2006 to 2010*

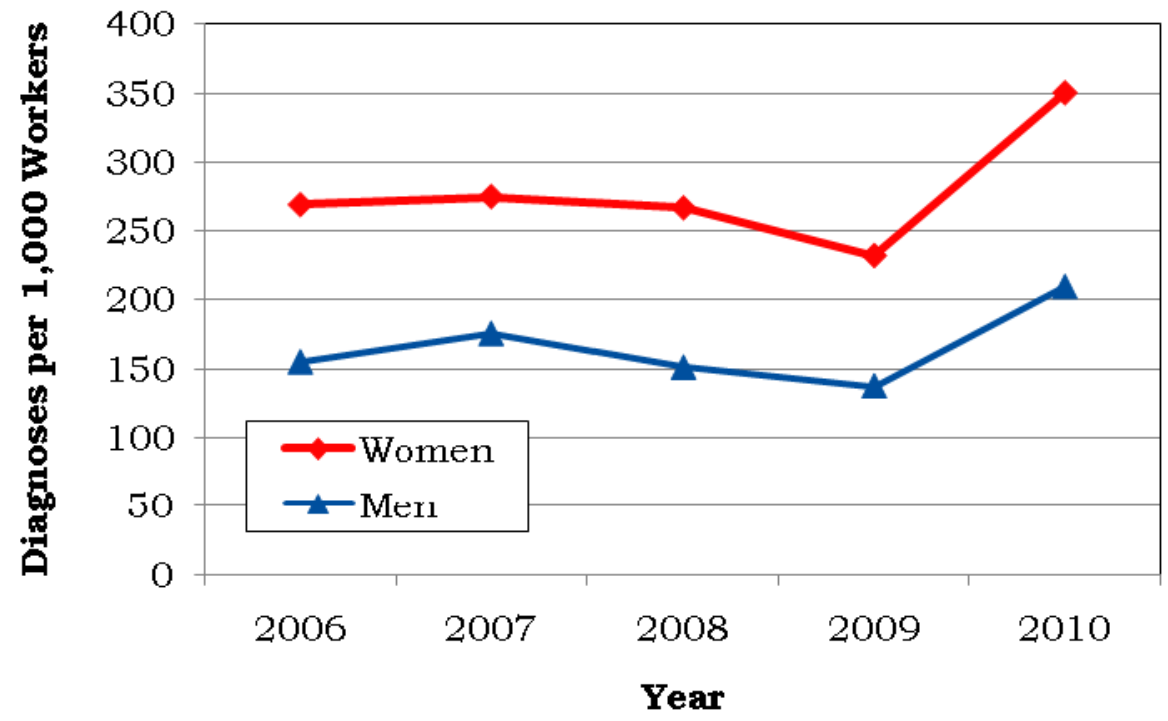

*Standardized to age distribution of 2000 U.S. population. 
Figure 14. Age-Adjusted Rates for Selected Diagnostic Categories Among Women and Men from 2006 to 2010*

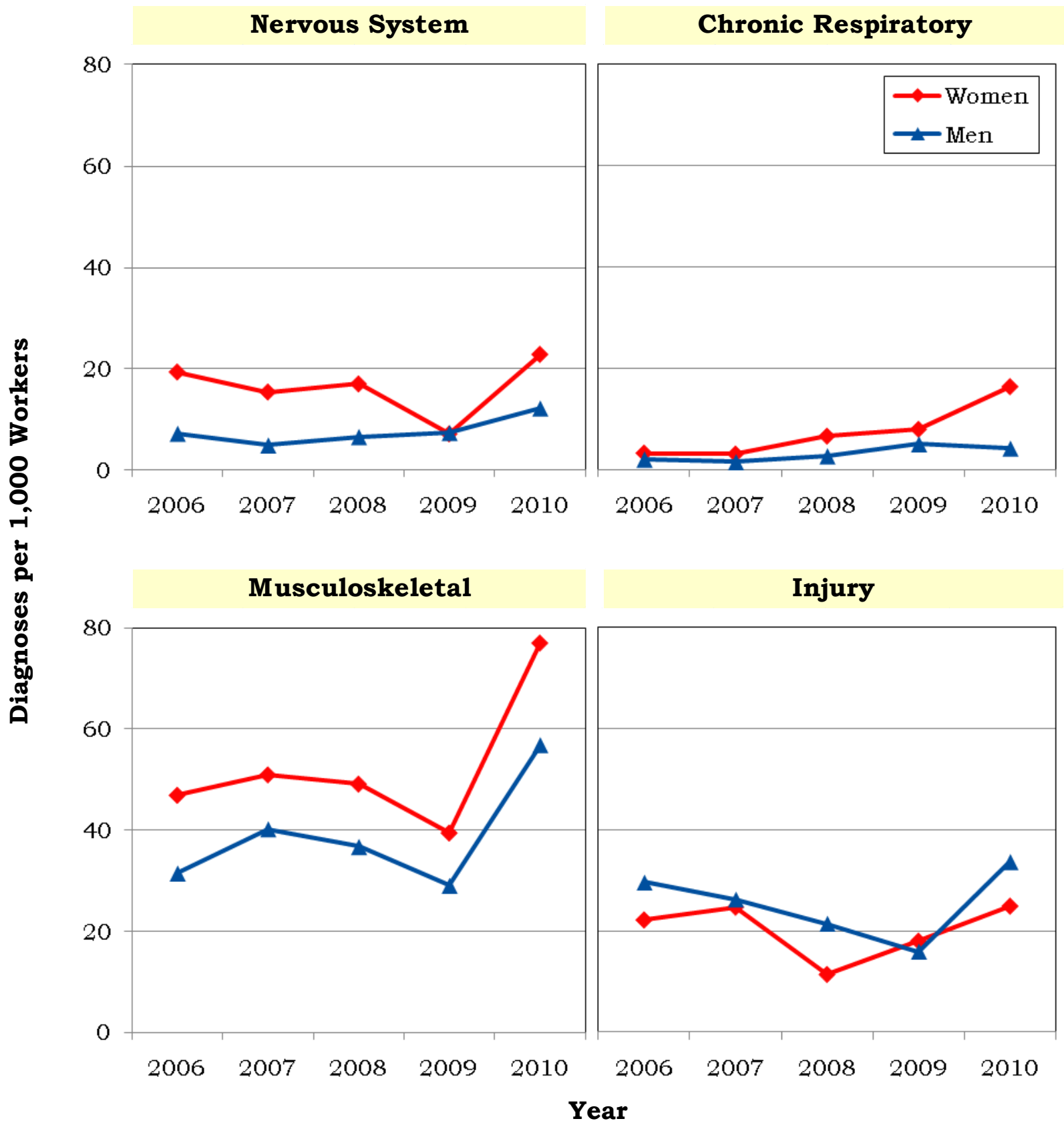

*Standardized to age distribution of 2000 U.S. population. 
Figure 15. Age-Adjusted Rates for All Diagnoses Combined Among Women and Men by Job Category from 2006 to 2010*

Professional

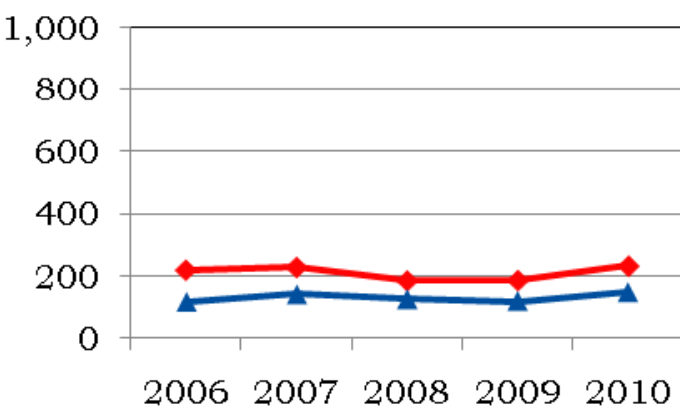

Administrative Support

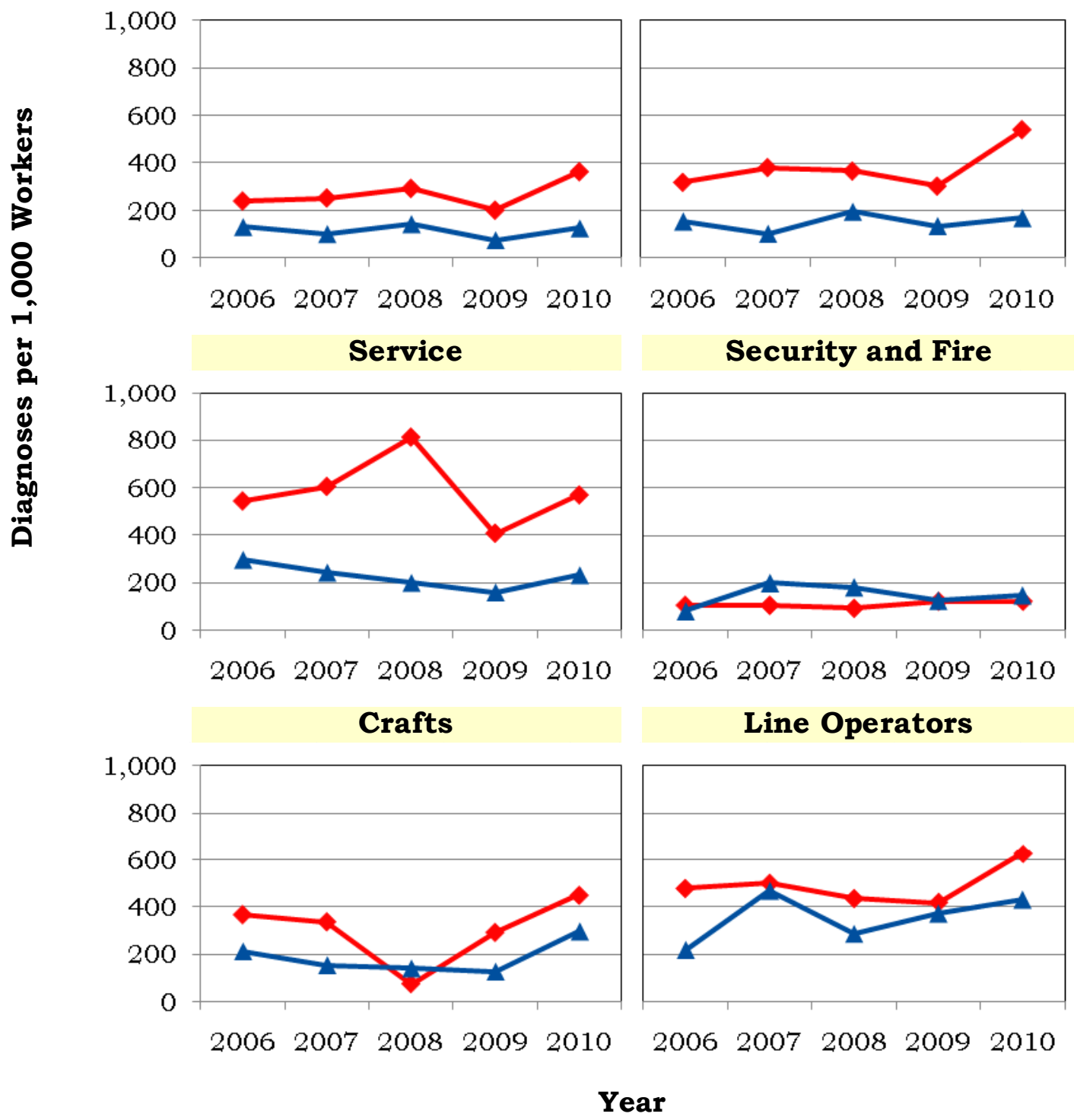

*Standardized to age distribution of 2000 U.S. population. 


\section{Sentinel Health Events for Occupations (SHEOs)}

An occupational sentinel health event (SHEO) is a disease, disability, or death that is likely to be occupationally related. Although sentinel health events may indicate an occupational exposure, many may result from nonoccupational exposures. Sentinel health events are therefore assessed in two categories:

Definite Sentinel Health Events: Diseases that are unlikely to occur in the absence of an occupational exposure (e.g., asbestosis).

Possible Sentinel Health Events: Diseases that may be occupational but can also occur in the absence of an occupational exposure (e.g., lung cancer or carpal tunnel syndrome).

Figure 16. Characteristics of SHEOs by Gender

\begin{tabular}{|l|c|c|c|c|}
\hline \multirow{2}{*}{} & \multicolumn{2}{|c|}{$\begin{array}{c}\text { Total Number of } \\
\text { SHEO Diagnoses }\end{array}$} & \multicolumn{2}{c|}{$\begin{array}{c}\text { Total Number of } \\
\text { Days Absent }\end{array}$} \\
\cline { 2 - 5 } & Men & W omen & Men & W omen \\
\hline Definite & 0 & 0 & 0 & 0 \\
\hline Possible & 10 & 7 & 779 & 184 \\
\hline Total & 10 & 7 & 779 & 184 \\
\hline
\end{tabular}

Figure 17. SHEO Diagnoses by Gender

\begin{tabular}{|l|c|c|}
\hline \multirow{2}{*}{ Diagnoses } & \multicolumn{2}{c|}{ Gender } \\
\cline { 2 - 3 } & Women & M en \\
\hline Carpal Tunnel Syndrome & 5 & 3 \\
\hline Other Conditions & 2 & 7 \\
\hline
\end{tabular}


Occupational Safety and Health Administration (OSHA)-Recordable Events

Figure 18. OSHA-Recordable Events by Gender and Age

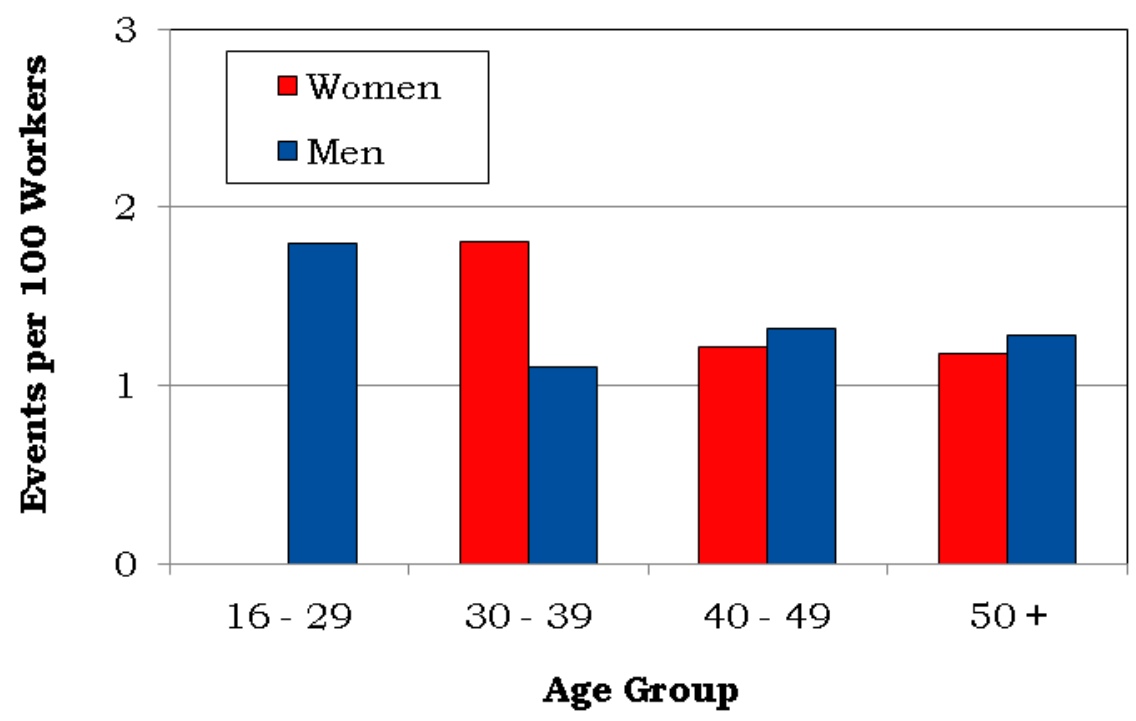

Figure 19. OSHA-Recordable Events by Job Category and Gender

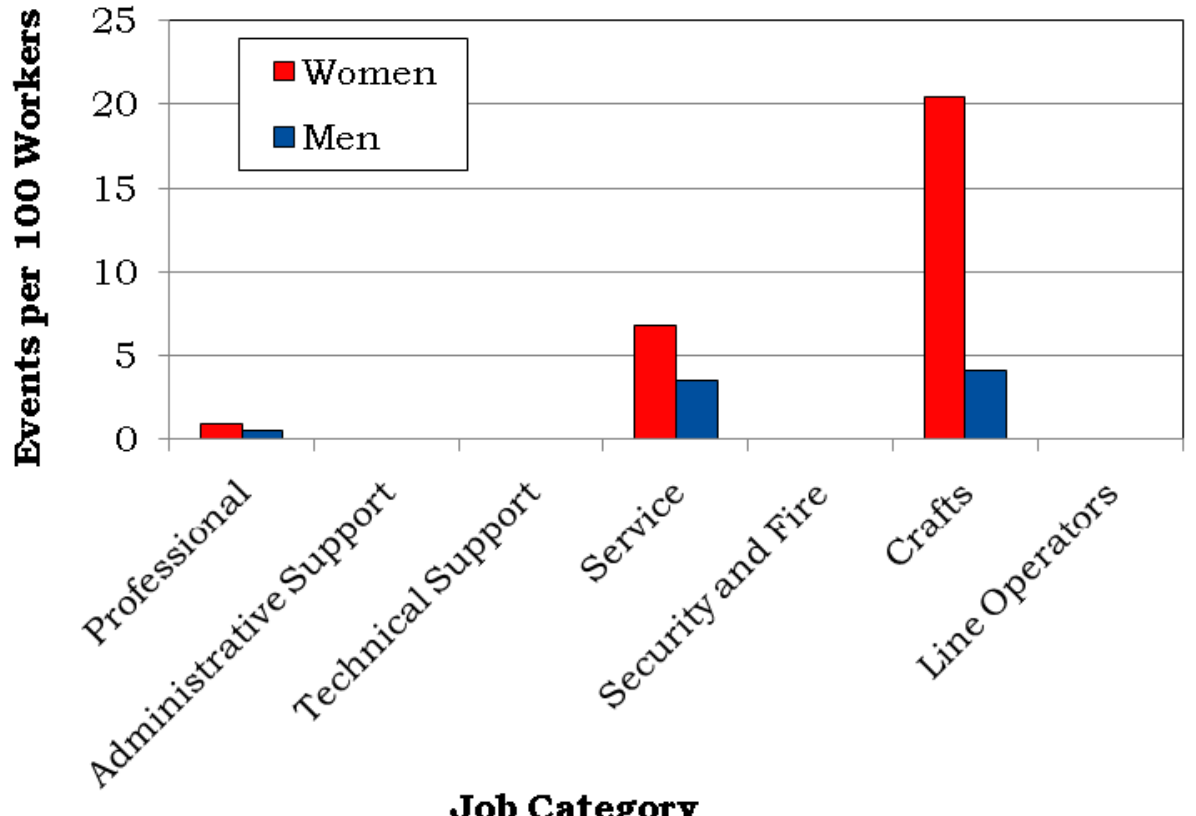

Job Category 


\section{Diagnostic and Accident Categories for OSHA-Recordable Events}

Figure 20. OSHA-Recordable Diagnoses by Diagnostic Category and Gender

\begin{tabular}{|l|c|c|}
\hline \multirow{2}{*}{ Diagnostic Category } & \multicolumn{2}{c|}{ Gender } \\
\cline { 2 - 3 } & W omen & M en \\
\hline Musculoskeletal & $\mathbf{1}$ & $\mathbf{1 6}$ \\
\hline Nervous System & $\mathbf{0}$ & $\mathbf{2}$ \\
\hline Respiratory & $\mathbf{0}$ & $\mathbf{4}$ \\
\hline Unspecified Symptoms & $\mathbf{1}$ & $\mathbf{4}$ \\
\hline Injury & $\mathbf{3 0}$ & $\mathbf{5 8}$ \\
\hline Fractures - Neck, Trunk & $\mathbf{0}$ & $\mathbf{1}$ \\
\hline Fractures - Upper Limb & $\mathbf{1}$ & $\mathbf{3}$ \\
\hline Fractures - Lower Limb & $\mathbf{0}$ & $\mathbf{1}$ \\
\hline Dislocations & $\mathbf{1}$ & $\mathbf{2}$ \\
\hline Back Sprains \& Strains & $\mathbf{1}$ & $\mathbf{6}$ \\
\hline Other Sprains \& Strains & $\mathbf{5}$ & $\mathbf{1 0}$ \\
\hline Intracranial Injuries & $\mathbf{1}$ & $\mathbf{2}$ \\
\hline Open Wounds - Head, Neck, Trunk & $\mathbf{2}$ & $\mathbf{1}$ \\
\hline Open Wounds - Upper Limb & $\mathbf{2}$ & $\mathbf{5}$ \\
\hline Open Wounds - Lower Limb & $\mathbf{0}$ & $\mathbf{2}$ \\
\hline Superficial Injuries & $\mathbf{2}$ & $\mathbf{3}$ \\
\hline Bruises & $\mathbf{1 3}$ & $\mathbf{9}$ \\
\hline Crushing Injuries & $\mathbf{0}$ & $\mathbf{1}$ \\
\hline Foreign Bodies Entering Orifice & $\mathbf{1}$ & $\mathbf{5}$ \\
\hline Burns & $\mathbf{0}$ & $\mathbf{2}$ \\
\hline Unspecified Injuries & $\mathbf{0}$ \\
\hline Adverse Reactions to Non-Medical Substances & $\mathbf{4}$ \\
\hline Adverse Reactions to External Causes & $\mathbf{1}$ \\
\hline
\end{tabular}


Figure 21. OSHA-Recordable Accidents by Type and Gender

\begin{tabular}{|l|c|c|}
\hline \multirow{2}{*}{\multicolumn{1}{c|}{ Accident Category }} & \multicolumn{2}{c|}{ Gender } \\
\cline { 2 - 3 } & Women & Men \\
\cline { 2 - 3 } & Number of & Number of \\
Accidents & Accidents \\
\hline Motor Vehicle Traffic & $\mathbf{0}$ & $\mathbf{1}$ \\
\hline Non Motor Vehicle & $\mathbf{0}$ & $\mathbf{1}$ \\
\hline Poisoning - Non-Medicinal & $\mathbf{0}$ & $\mathbf{1}$ \\
\hline Falls & $\mathbf{1 2}$ & $\mathbf{6}$ \\
\hline Natural/Environmental Factors & $\mathbf{0}$ & $\mathbf{4}$ \\
\hline Submersion/Suffocation/Foreign Bodies & $\mathbf{1}$ & $\mathbf{3}$ \\
\hline Other Accidents & $\mathbf{3}$ & $\mathbf{2 9}$ \\
\hline Struck by an Object & $\mathbf{2}$ & $\mathbf{1 2}$ \\
\hline Caught Between Objects & $\mathbf{1}$ & $\mathbf{0}$ \\
\hline Cutting/Piercing Instrument/Object & $\mathbf{0}$ & $\mathbf{2}$ \\
\hline Hot, Corrosive, or Caustic Material/Steam & $\mathbf{0}$ & $\mathbf{2}$ \\
\hline Overexertion/Strenuous Movements & $\mathbf{0}$ & $\mathbf{1 3}$ \\
\hline Total & $\mathbf{1 6}$ & $\mathbf{4 5}$ \\
\hline
\end{tabular}




\section{Rates of OSHA-Recordable Events}

Figure 22. OSHA-Recordable Rates by Age and Job Categories Among Women, All Diagnoses Combined

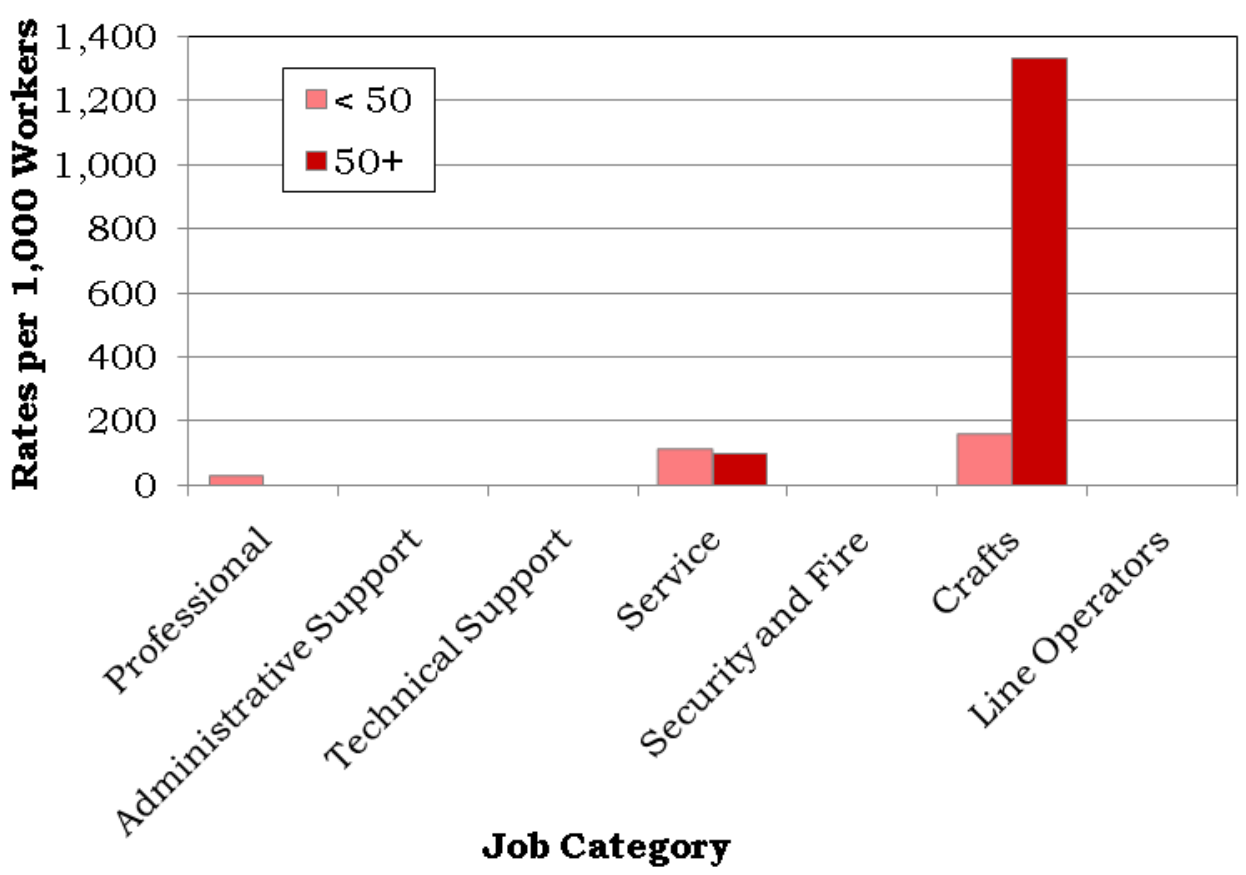

Figure 23. OSHA-Recordable Rates by Age and Job Categories Among Men, All Diagnoses Combined

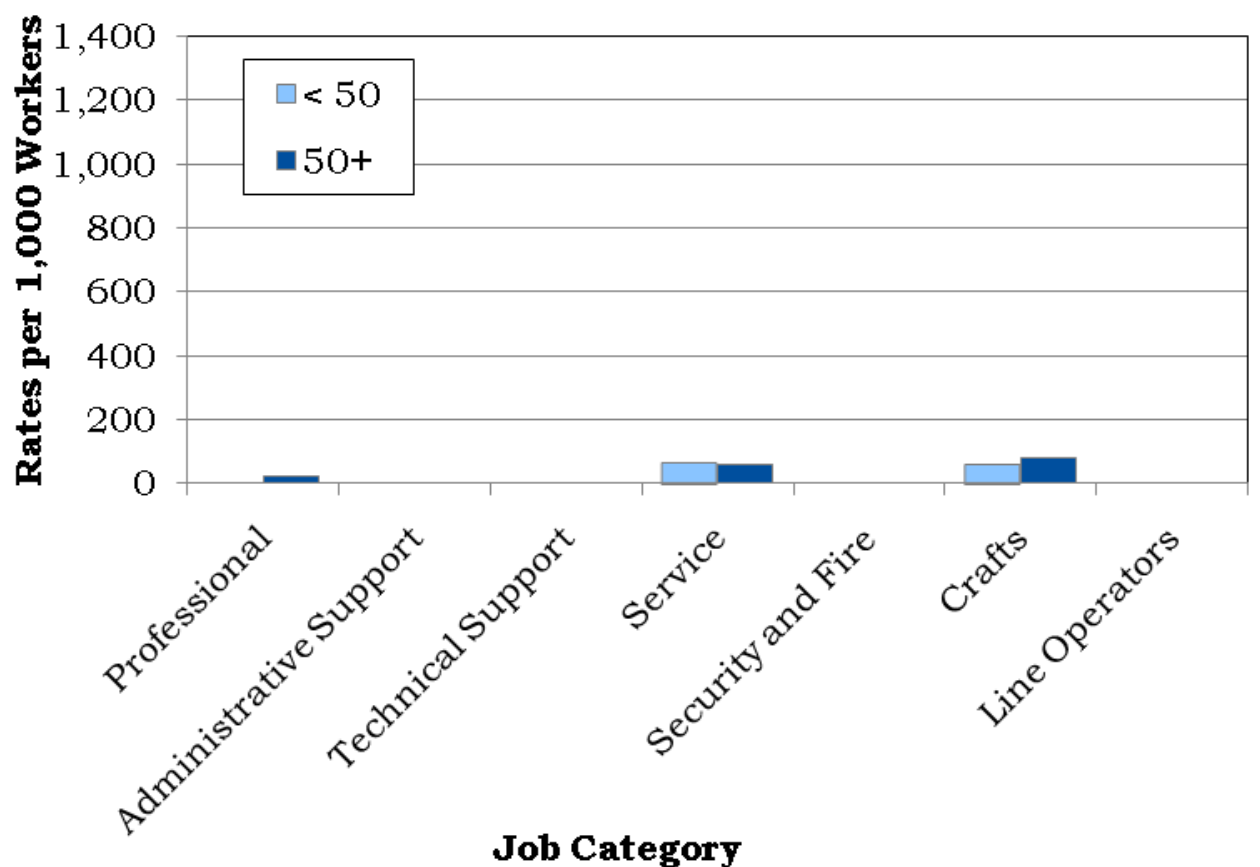

Job Category 


\section{Time Trends for OSHA-Recordable Events}

Figure 24. Age-Adjusted Rates for All OSHA-Recordable Diagnoses Combined Among Women and Men by Job Category from 2006 to 2010*

Professional

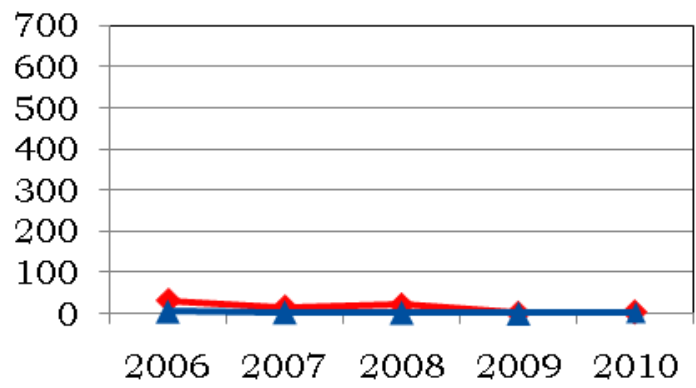

Administrative Sup port
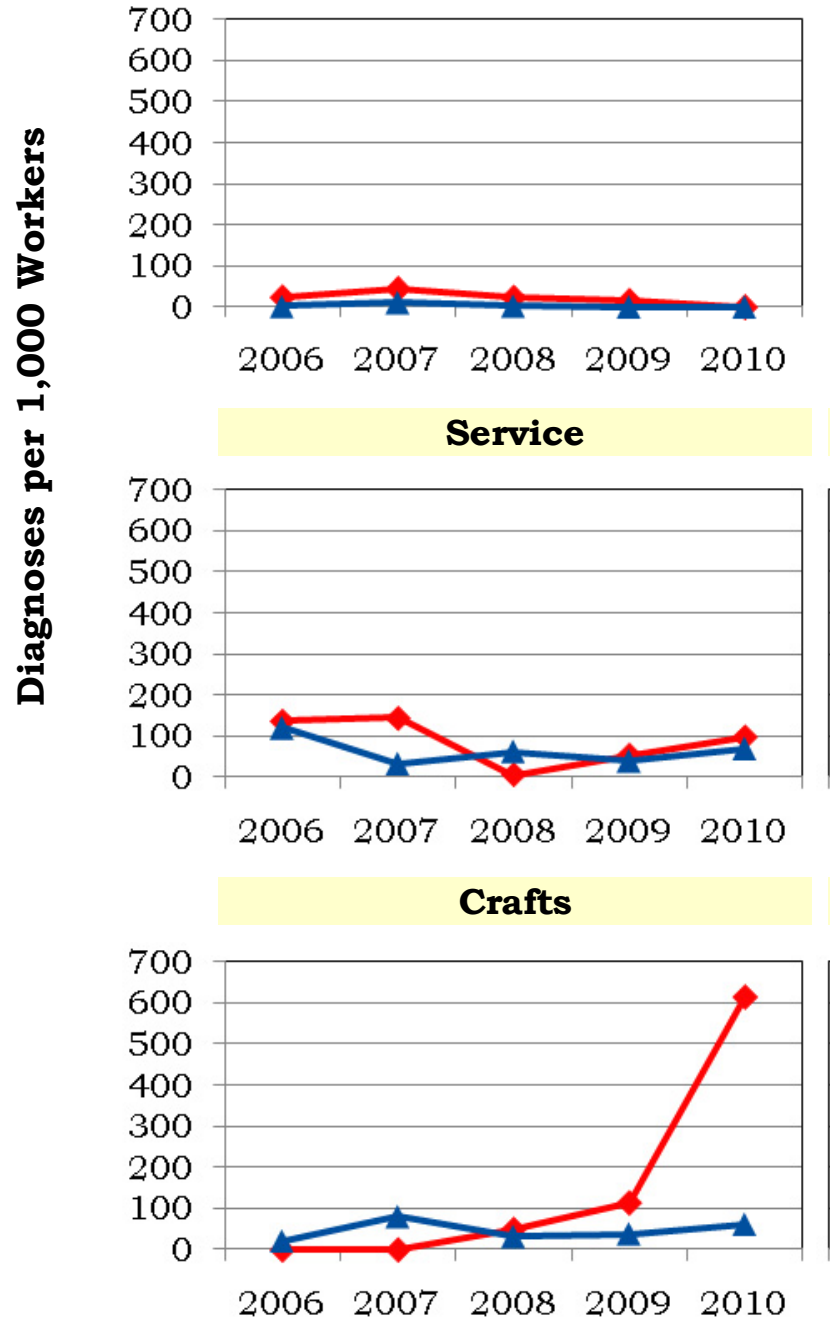

Technical Support

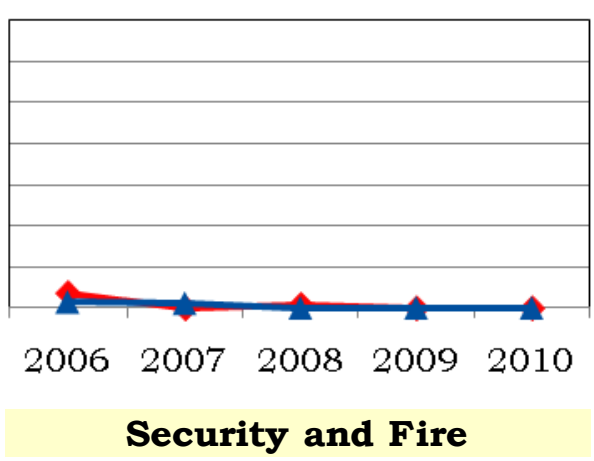

Security and Fire
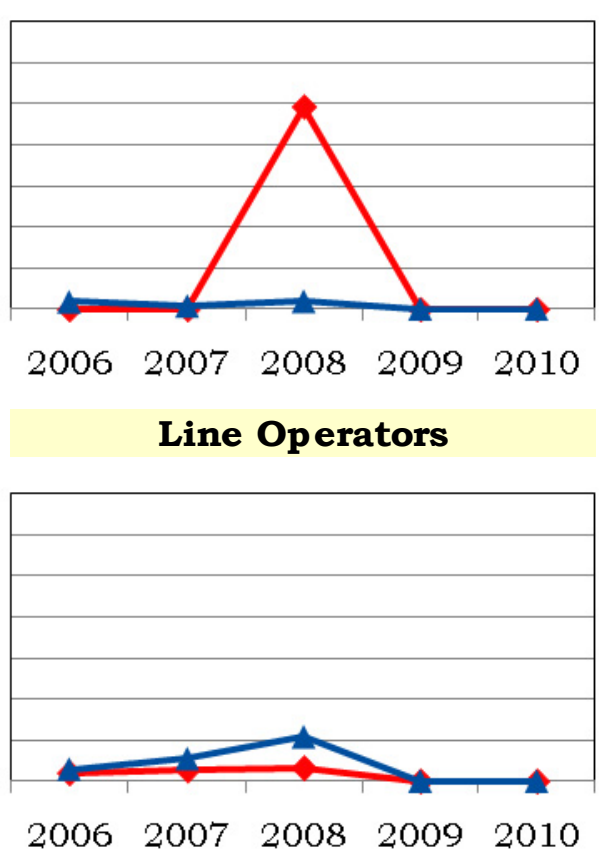

\section{Year}

*Standardized to age distribution of 2000 U.S. population. 
Appendices 
Y-12 National Security Complex 2010

Absence Data

Appendix A. Work Force by Gender, Age, and Job Category

\begin{tabular}{|c|c|c|c|c|c|c|c|c|c|c|c|}
\hline \multirow{3}{*}{ Job Category } & \multicolumn{5}{|c|}{ Women } & \multicolumn{5}{|c|}{ Men } & \multirow[b]{3}{*}{ TOTAL } \\
\hline & \multicolumn{4}{|c|}{ Age Group } & \multirow[b]{2}{*}{ TOTAL } & \multicolumn{4}{|c|}{ Age Group } & \multirow[b]{2}{*}{ TOTAL } & \\
\hline & $16-29$ & 30 - 39 & $40-49$ & $50+$ & & $16-29$ & $30-39$ & $40-49$ & $50+$ & & \\
\hline Professional & 51 & 60 & 152 & 226 & 489 & 125 & 186 & 365 & 965 & 1,641 & 2,130 \\
\hline Administrative Support & 33 & 61 & 158 & 293 & 545 & 29 & 52 & 73 & 197 & 351 & 896 \\
\hline Technical Support & 7 & 16 & 25 & 49 & 97 & 20 & 24 & 35 & 64 & 143 & 240 \\
\hline Service & 7 & 5 & 25 & 52 & 89 & 50 & 67 & 91 & 167 & 375 & 464 \\
\hline Security and Fire & 2 & 7 & 20 & 18 & 47 & 7 & 35 & 40 & 50 & 132 & 179 \\
\hline Crafts & 6 & 12 & 14 & 12 & 44 & 91 & 130 & 175 & 433 & 829 & 873 \\
\hline Line Operators & 0 & 5 & 16 & 26 & 47 & 11 & 51 & 52 & 74 & 188 & 235 \\
\hline TOTAL & 106 & 166 & 410 & 676 & 1,358 & 333 & 545 & 831 & 1,950 & 3,659 & 5,017 \\
\hline
\end{tabular}

Appendix B. Age Distribution of the Work Force by Gender

\begin{tabular}{|l|r|r|r|r|r|r|r|r|}
\hline \multirow{3}{*}{ Year } & \multicolumn{4}{|c|}{ Women } & \multicolumn{4}{c|}{ Men } \\
\cline { 2 - 9 } & \multicolumn{1}{|c|}{ Percent Distribution by Age Group } & \multicolumn{1}{c|}{ Percent Distribution by Age Group } \\
\cline { 2 - 9 } & $\mathbf{1 6}-\mathbf{2 9}$ & $\mathbf{3 0}-\mathbf{3 9}$ & $\mathbf{4 0}-\mathbf{4 9}$ & $\mathbf{5 0}+$ & $\mathbf{1 6}-\mathbf{2 9}$ & $\mathbf{3 0}-\mathbf{3 9}$ & $\mathbf{4 0}-\mathbf{4 9}$ & $\mathbf{5 0}+$ \\
\hline $\mathbf{1 9 9 8}$ & 3.53 & 25.56 & 44.17 & 26.74 & 1.38 & 14.26 & 39.96 & 44.40 \\
\hline $\mathbf{1 9 9 9}$ & 3.21 & 23.11 & 45.31 & 28.37 & 1.30 & 14.80 & 38.78 & 45.12 \\
\hline $\mathbf{2 0 0 0}$ & 2.93 & 21.13 & 45.34 & 30.61 & 1.63 & 13.25 & 38.59 & 46.54 \\
\hline $\mathbf{2 0 0 1}$ & 2.02 & 19.48 & 45.70 & 32.80 & 2.09 & 11.10 & 36.21 & 50.60 \\
\hline $\mathbf{2 0 0 2}$ & 4.95 & 18.75 & 42.91 & 33.38 & 5.79 & 12.13 & 32.42 & 49.66 \\
\hline $\mathbf{2 0 0 3}$ & 6.36 & 16.02 & 42.99 & 34.64 & 5.91 & 11.74 & 30.31 & 52.04 \\
\hline $\mathbf{2 0 0 4}$ & 6.77 & 14.59 & 40.00 & 38.65 & 6.57 & 11.68 & 27.69 & 54.06 \\
\hline $\mathbf{2 0 0 5}$ & 6.92 & 12.84 & 37.82 & 42.43 & 7.90 & 11.24 & 26.06 & 54.81 \\
\hline $\mathbf{2 0 0 6}$ & 7.44 & 13.01 & 35.04 & 44.51 & 7.83 & 11.37 & 25.43 & 55.37 \\
\hline $\mathbf{2 0 0 7}$ & 6.89 & 11.81 & 33.69 & 47.62 & 7.28 & 11.96 & 24.88 & 55.88 \\
\hline $\mathbf{2 0 0 8}$ & 7.26 & 11.75 & 32.19 & 48.80 & 8.07 & 12.81 & 24.17 & 54.94 \\
\hline $\mathbf{2 0 0 9}$ & 7.57 & 11.93 & 32.19 & 48.32 & 8.93 & 14.15 & 24.84 & 52.08 \\
\hline $\mathbf{2 0 1 0}$ & 7.81 & 12.22 & 30.19 & 49.78 & 9.10 & 14.89 & 22.71 & 53.29 \\
\hline
\end{tabular}


Y-12 National Security Complex 2010

Absence Data

Appendix C. Total Number of Workers Who Reported at Least One Absence by Gender, Age, and Job Category*

\begin{tabular}{|c|c|c|c|c|c|c|c|c|c|c|c|}
\hline \multirow{3}{*}{ Job Category } & \multicolumn{5}{|c|}{ Women } & \multicolumn{5}{|c|}{ Men } & \multirow[b]{3}{*}{ TOTAL } \\
\hline & \multicolumn{4}{|c|}{ Age Group } & \multirow[b]{2}{*}{ TOTAL } & \multicolumn{4}{|c|}{ Age Group } & \multirow[b]{2}{*}{ TOTAL } & \\
\hline & $16-29$ & 30 - 39 & $40-49$ & $50+$ & & 16 - 29 & 30 - 39 & $40-49$ & $50+$ & & \\
\hline Professional & 2 & 8 & 26 & 36 & 72 & 2 & 21 & 27 & 131 & 181 & 253 \\
\hline Administrative Support & 5 & 11 & 27 & 70 & 113 & 1 & 3 & 5 & 31 & 40 & 153 \\
\hline Technical Support & 0 & 4 & 8 & 15 & 27 & 1 & 4 & 6 & 9 & 20 & 47 \\
\hline Service & 2 & 0 & 6 & 24 & 32 & 6 & 7 & 9 & 32 & 54 & 86 \\
\hline Security and Fire & 0 & 1 & 1 & 3 & 5 & 1 & 2 & 2 & 9 & 14 & 19 \\
\hline Crafts & 1 & 3 & 3 & 3 & 10 & 10 & 15 & 22 & 92 & 139 & 149 \\
\hline Line Operators & 0 & 4 & 7 & 9 & 20 & 1 & 11 & 12 & 23 & 47 & 67 \\
\hline TOTAL & 10 & 31 & 78 & 160 & 279 & 22 & 63 & 83 & 327 & 495 & 774 \\
\hline
\end{tabular}

*Only those job categories and gender/age combinations with at least one absence appear in this table.

Appendix D. Total Number of Absences by Gender, Age, and Job Category*

\begin{tabular}{|c|c|c|c|c|c|c|c|c|c|c|c|}
\hline \multirow{3}{*}{ Job Category } & \multicolumn{5}{|c|}{ Women } & \multicolumn{5}{|c|}{ Men } & \multirow[b]{3}{*}{ TOTAL } \\
\hline & \multicolumn{4}{|c|}{ Age Group } & \multirow[b]{2}{*}{ TOTAL } & \multicolumn{4}{|c|}{ Age Group } & \multirow[b]{2}{*}{ TOTAL } & \\
\hline & $16-29$ & 30 - 39 & $40-49$ & $50+$ & & $16-29$ & 30 - 39 & $40-49$ & $50+$ & & \\
\hline Professional & 2 & 8 & 31 & 44 & 85 & 2 & 24 & 31 & 154 & 211 & 296 \\
\hline Administrative Support & 6 & 12 & 37 & 81 & 136 & 1 & 4 & 6 & 31 & 42 & 178 \\
\hline Technical Support & 0 & 6 & 9 & 24 & 39 & 1 & 5 & 7 & 9 & 22 & 61 \\
\hline Service & 4 & 0 & 10 & 29 & 43 & 8 & 9 & 11 & 33 & 61 & 104 \\
\hline Security and Fire & 0 & 1 & 1 & 3 & 5 & 1 & 2 & 2 & 9 & 14 & 19 \\
\hline Crafts & 2 & 5 & 4 & 5 & 16 & 12 & 21 & 24 & 118 & 175 & 191 \\
\hline Line Operators & 0 & 4 & 8 & 15 & 27 & 1 & 13 & 19 & 26 & 59 & 86 \\
\hline TOTAL & 14 & 36 & 100 & 201 & 351 & 26 & 78 & 100 & 380 & 584 & 935 \\
\hline
\end{tabular}

*Only those job categories and gender/age combinations with at least one absence appear in this table. 
Y-12 National Security Complex 2010

Absence Data

Appendix E. Distribution of the Number of Calendar Days Missed per Absence by Gender and Age*

\begin{tabular}{|c|c|c|c|c|c|c|c|c|c|c|c|}
\hline \multirow{3}{*}{$\begin{array}{c}\text { Number of } \\
\text { Calendar Days }\end{array}$} & \multicolumn{5}{|c|}{ Women } & \multicolumn{5}{|c|}{ Men } & \multirow[b]{3}{*}{ TOTAL } \\
\hline & \multicolumn{4}{|c|}{ Age Group } & \multirow[b]{2}{*}{ TOTAL } & \multicolumn{4}{|c|}{ Age Group } & \multirow[b]{2}{*}{ TOTAL } & \\
\hline & $16-29$ & $30-39$ & $40-49$ & $50+$ & & $16-29$ & 30 - 39 & $40-49$ & $50+$ & & \\
\hline$<15$ & 4 & 16 & 45 & 87 & 152 & 5 & 26 & 41 & 131 & 203 & 355 \\
\hline $15-28$ & 4 & 8 & 20 & 32 & 64 & 7 & 20 & 19 & 74 & 120 & 184 \\
\hline $29-42$ & 1 & 3 & 7 & 23 & 34 & 5 & 10 & 11 & 42 & 68 & 102 \\
\hline $43-56$ & 2 & 2 & 5 & 15 & 24 & 1 & 8 & 10 & 30 & 49 & 73 \\
\hline $57-91$ & 3 & 5 & 16 & 15 & 39 & 4 & 6 & 12 & 43 & 65 & 104 \\
\hline $92-182$ & 0 & 2 & 6 & 22 & 30 & 4 & 7 & 6 & 51 & 68 & 98 \\
\hline $183+$ & 0 & 0 & 1 & 7 & 8 & 0 & 1 & 1 & 9 & 11 & 19 \\
\hline TOTAL & 14 & 36 & 100 & 201 & 351 & 26 & 78 & 100 & 380 & 584 & 935 \\
\hline
\end{tabular}

*Only those gender/age combinations with at least one absence appear in this table. 
Y-12 National Security Complex 2010

Absence Data

Appendix F. Distribution of the Number of Calendar Days Missed per Absence by Gender and Job Category*

Women

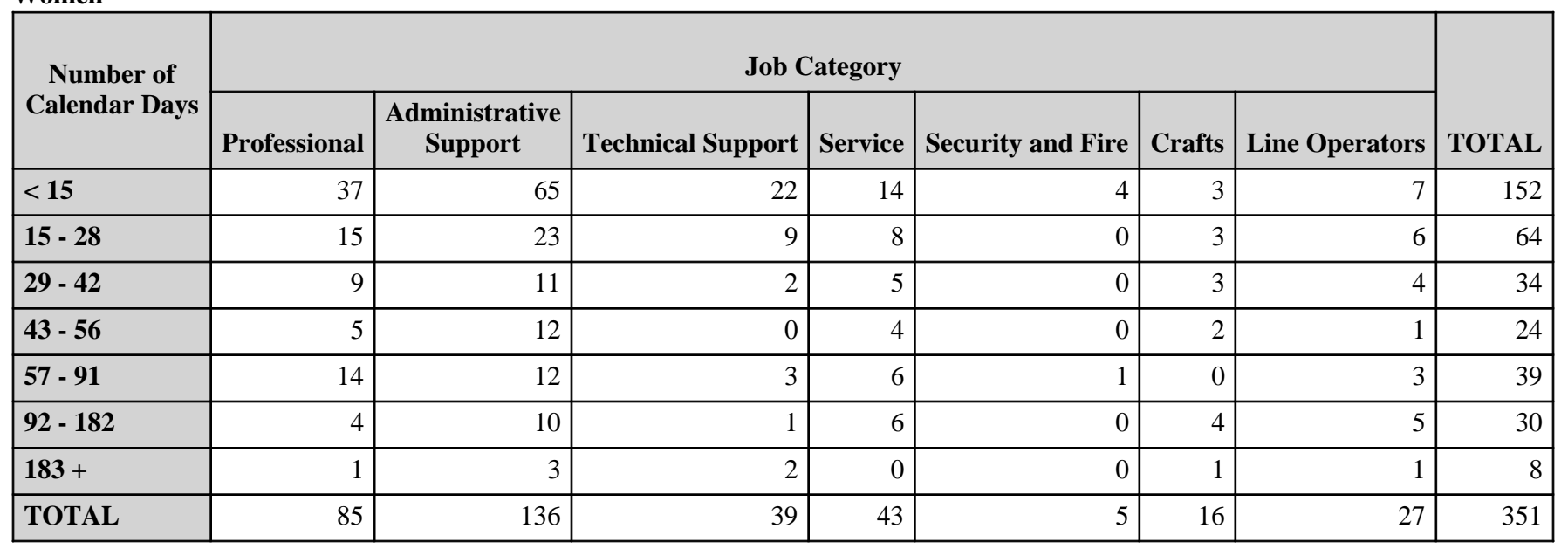

Men

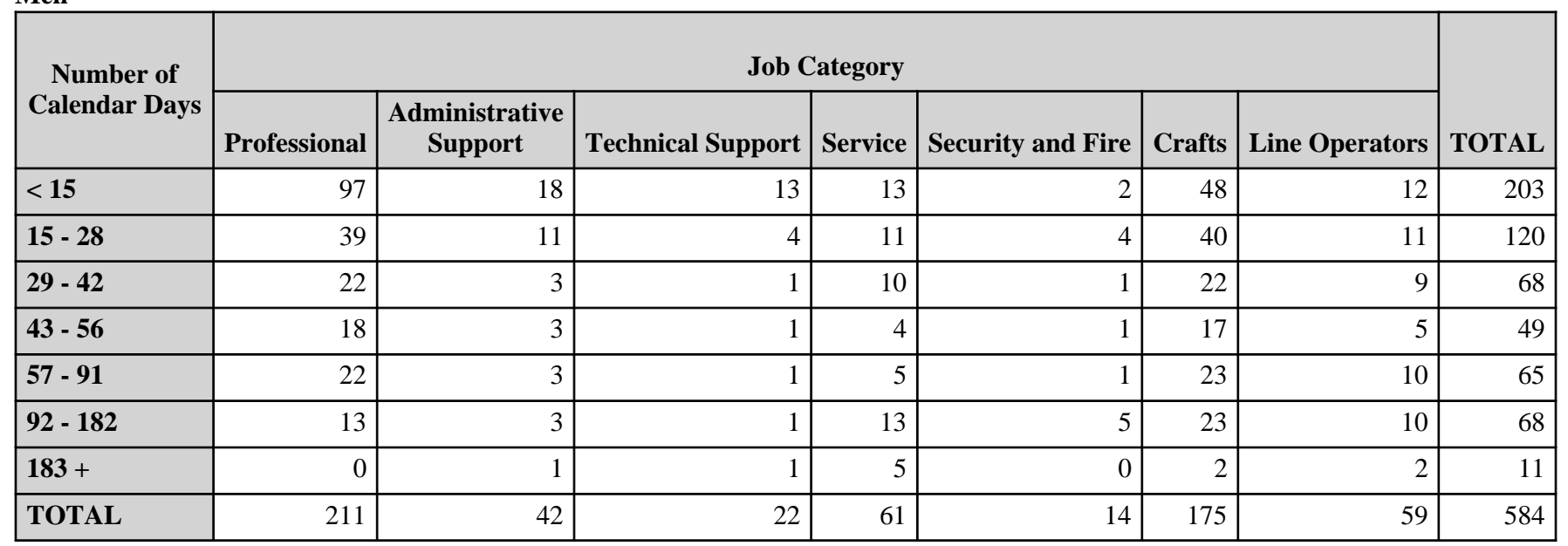

*Only those gender/job category combinations with at least one absence appear in this table. 


\section{Y-12 National Security Complex 2010}

\section{Absence Data}

Appendix G. Number of Diagnoses in Each Diagnostic Category by Gender and Age*

\begin{tabular}{|c|c|c|c|c|c|c|}
\hline & & \multicolumn{5}{|c|}{ Women } \\
\hline & & \multicolumn{4}{|c|}{ Age Group } & \multirow[b]{2}{*}{ TOTAL } \\
\hline & & $16-29$ & $30-39$ & $40-49$ & $50+$ & \\
\hline Diagnostic Category & ICD-9-CM Code & \multirow[b]{2}{*}{0} & \multirow[b]{2}{*}{1} & \multirow[b]{2}{*}{5} & \multirow[b]{2}{*}{6} & \multirow[b]{2}{*}{12} \\
\hline INFECTIOUS \& PARASITIC DISEASES (DIS) & $001-139$ & & & & & \\
\hline -Intestinal Infectious Dis & 001-009 & 0 & 0 & 1 & 3 & 4 \\
\hline -Other Bacterial Dis & \begin{tabular}{|l|}
$030-041$ \\
\end{tabular} & 0 & 1 & 2 & 0 & 3 \\
\hline -Viral Dis with Exanthem & 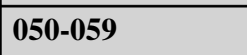 & 0 & 0 & 0 & 1 & 1 \\
\hline -Other Viral Dis \& Chlamydiae & 070-079 & 0 & 0 & 1 & 1 & 2 \\
\hline -Syphilis \& Other Venereal Dis & 090-099 & 0 & 0 & 0 & 1 & 1 \\
\hline -Other Infections \& Parasitic Dis & $130-136$ & 0 & 0 & 1 & 0 & 1 \\
\hline -Late Effects & \begin{tabular}{|l|}
$137-139$ \\
\end{tabular} & 0 & 0 & 0 & 0 & 0 \\
\hline MALIGNANT NEOPLASMS & $\begin{array}{l}140-208, \\
209.0-209.3 \\
230-234\end{array}$ & 0 & 0 & 2 & 6 & 8 \\
\hline -Digestive \& Peritoneal & $150-159$ & 0 & 0 & 0 & 2 & 2 \\
\hline -Respiratory \& Intrathoracic & $160-165$ & 0 & 0 & 0 & 0 & $\overline{0}$ \\
\hline -Bone, Connective Tissue, Skin & $170-173,176$ & 0 & 0 & 0 & 0 & 0 \\
\hline -Breast & 174-175 & 0 & 0 & 1 & 2 & 3 \\
\hline -Genitourinary & 179-189 & 0 & 0 & 0 & 1 & 1 \\
\hline -Nervous System & 190-192 & 0 & 0 & 0 & 0 & 0 \\
\hline -Endocrine & $\begin{array}{l}\text { 193-194, } \\
209.0-209.3\end{array}$ & 0 & 0 & 0 & 1 & 1 \\
\hline -Lymphatic \& Hematopoietic & $200-208$ & 0 & 0 & 0 & 0 & 0 \\
\hline -Carcinoma in situ & $230-234$ & 0 & 0 & 1 & 0 & 1 \\
\hline BENIGN \& UNCERTAIN NEOPLASMS & $\begin{array}{l}209.4-209.6 \\
210-229,235-239\end{array}$ & 1 & 2 & 2 & 2 & 7 \\
\hline ENDOCRINE/METABOLIC/IMMUNITY & $240-279$ & 0 & 1 & 1 & 8 & 10 \\
\hline -Thyroid Gland Disorders & $240-246$ & 0 & 0 & 0 & 2 & 2 \\
\hline -Other Endocrine Gland Dis & $249-259$ & 0 & 0 & 0 & 4 & 4 \\
\hline -Other Metabolic \& Immunity Disorders & $270-279$ & 0 & 1 & 1 & 2 & 4 \\
\hline BLOOD \& BLOOD-FORMING ORGANS & $280-289$ & 0 & 0 & 0 & 2 & 2 \\
\hline MENTAL DISORDERS & $290-319$ & 3 & 1 & 5 & 3 & 12 \\
\hline -Psychoses & $290-299$ & 1 & 1 & 1 & 1 & 4 \\
\hline -Non-Psychotic Disorders & $300-302,306-316$ & 2 & 0 & 3 & 2 & 7 \\
\hline -Alcohol Dependence & 303 & 0 & 0 & 0 & 0 & 0 \\
\hline -Drug Dependence & $304-305$ & 0 & 0 & 1 & 0 & 1 \\
\hline NERVOUS SYSTEM (NS) \& SENSE ORGANS & 320-389 & 0 & 3 & 13 & 21 & 37 \\
\hline -Organic Sleep Disorders & 327 & 0 & 0 & 2 & 0 & 2 \\
\hline -Hereditary/Degenerative Central NS Dis & 330-337 & 0 & 0 & 1 & 0 & 1 \\
\hline -Pain & 338 & 0 & 0 & 0 & 0 & 0 \\
\hline -Other Disorders of Central NS & $340-349$ & 0 & 3 & 1 & 4 & 8 \\
\hline
\end{tabular}

(Continued)

*Only those diagnostic categories and gender/age combinations with at least one occurrence appear in this table. 


\section{Y-12 National Security Complex 2010}

\section{Absence Data}

Appendix G. Number of Diagnoses in Each Diagnostic Category by Gender and Age*

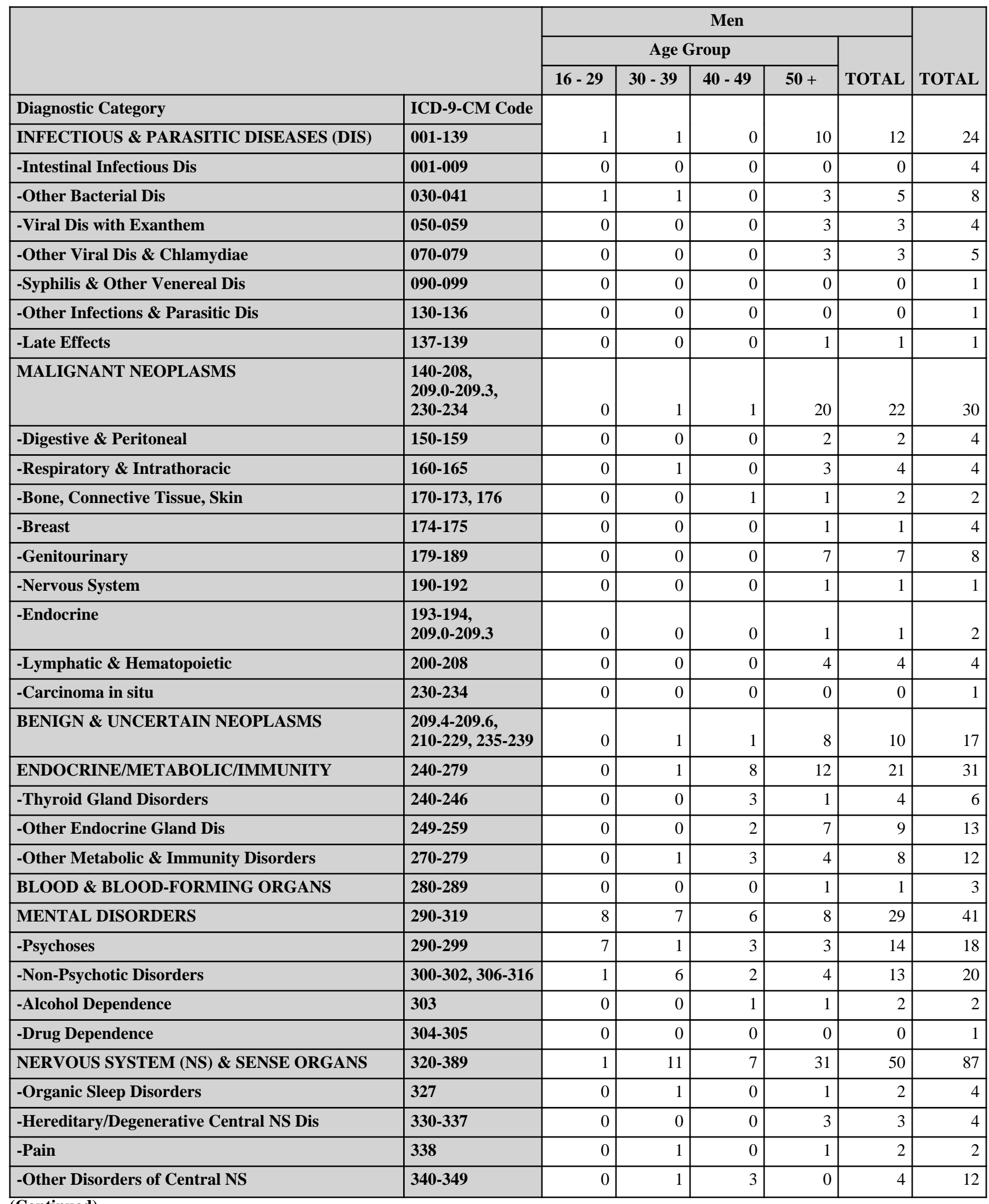

(Continued)

*Only those diagnostic categories and gender/age combinations with at least one occurrence appear in this table. 


\section{Y-12 National Security Complex 2010}

\section{Absence Data}

Appendix G. Number of Diagnoses in Each Diagnostic Category by Gender and Age*

\begin{tabular}{|c|c|c|c|c|c|c|}
\hline & & \multicolumn{5}{|c|}{ Women } \\
\hline & & \multicolumn{4}{|c|}{ Age Group } & \multirow[b]{2}{*}{ TOTAL } \\
\hline & & $16-29$ & $30-39$ & $40-49$ & $50+$ & \\
\hline Diagnostic Category & ICD-9-CM Code & & & & & \\
\hline -Disorders of Peripheral NS & $350-359$ & 0 & 0 & 5 & 6 & 11 \\
\hline -Disorders of Eye & $360-379$ & 0 & 0 & 1 & 5 & 6 \\
\hline -Diseases of Ear \& Mastoid & $380-389$ & 0 & 0 & 3 & 6 & 9 \\
\hline CIRCULATORY SYSTEM & $390-459$ & 1 & 0 & 4 & 18 & 23 \\
\hline -Hypertensive Dis & $401-405$ & 0 & 0 & 1 & 3 & 4 \\
\hline -Ischemic Heart Dis & $410-414$ & 0 & 0 & 0 & 5 & 5 \\
\hline -Dis of Pulmonary Circulation & $415-417$ & 0 & 0 & 0 & 1 & 1 \\
\hline -Other Heart Dis & $420-429$ & 0 & 0 & 0 & 1 & 1 \\
\hline -Cerebrovascular Dis & $430-438$ & 0 & 0 & 0 & 3 & 3 \\
\hline -Dis of Arteries \& Capillaries & $440-449$ & 0 & 0 & 0 & 2 & 2 \\
\hline -Dis of Veins, Lymphatics, Other & 451-459 & 1 & 0 & 3 & 3 & 7 \\
\hline RESPIRATORY SYSTEM & $460-519$ & 6 & 14 & 21 & 59 & 100 \\
\hline -Acute Respiratory Infections & $460-466$ & 3 & 6 & 8 & 21 & 38 \\
\hline -Other Dis Upper Respiratory Tract & $470-478$ & 1 & 5 & 4 & 10 & 20 \\
\hline -Pneumonia \& Influenza & $480-488$ & 1 & 1 & 1 & 5 & 8 \\
\hline -Chronic Obstructive Dis & $490-496$ & 1 & 1 & 6 & 20 & 28 \\
\hline -Other Respiratory Dis & $510-519$ & 0 & 1 & 2 & 3 & 6 \\
\hline DIGESTIVE SYSTEM & $520-579$ & 1 & 6 & 10 & 33 & 50 \\
\hline -Oral Cavity, Saliva Glands, Jaw & $520-529$ & 0 & 2 & 2 & 5 & 9 \\
\hline -Esophagus, Stomach, Duodenum & $530-538$ & 0 & 0 & 1 & 5 & 6 \\
\hline -Appendicitis & $540-543$ & 0 & 1 & 1 & 0 & 2 \\
\hline -Hernias & $550-553$ & 0 & 0 & 1 & 1 & 2 \\
\hline -Enteritis, Colitis & $555-558$ & 0 & 2 & 1 & 3 & 6 \\
\hline -Other Intestinal Dis & $560-569$ & 0 & 0 & 2 & 7 & 9 \\
\hline -Other Digestive Dis & $570-579$ & 1 & 1 & 2 & 12 & 16 \\
\hline GENITOURINARY SYSTEM & $580-629$ & 3 & 5 & 15 & 24 & 47 \\
\hline -Nephritis, Nephrosis & $580-589$ & 0 & 0 & 0 & 3 & 3 \\
\hline -Other Urinary Dis & $590-599$ & 0 & 1 & 1 & 4 & 6 \\
\hline -Male Genital Organ Dis & $600-608$ & 0 & 0 & 0 & 0 & 0 \\
\hline -Breast Disorders & 610-612 & 0 & 0 & 3 & 1 & 4 \\
\hline -Other Female Disorders & $617-629$ & 3 & 4 & 11 & 16 & 34 \\
\hline SKIN AND SUBCUTANEOUS TISSUE & $680-709$ & 0 & 0 & 2 & 2 & 4 \\
\hline -Infections & $680-686$ & 0 & 0 & 1 & 0 & 1 \\
\hline -Other Inflammatory Conditions & $690-698$ & 0 & 0 & 0 & 1 & 1 \\
\hline -Other & 700-709 & 0 & 0 & 1 & 1 & 2 \\
\hline $\begin{array}{l}\text { MUSCULOSKELETAL \& CONNECTIVE } \\
\text { TISSUE }\end{array}$ & 710-739 & 1 & 14 & 31 & 75 & 121 \\
\hline
\end{tabular}

(Continued)

*Only those diagnostic categories and gender/age combinations with at least one occurrence appear in this table. 


\section{Y-12 National Security Complex 2010}

\section{Absence Data}

Appendix G. Number of Diagnoses in Each Diagnostic Category by Gender and Age*

\begin{tabular}{|c|c|c|c|c|c|c|c|}
\hline & & \multicolumn{5}{|c|}{ Men } & \multirow[b]{3}{*}{ TOTAL } \\
\hline & & \multicolumn{4}{|c|}{ Age Group } & \multirow[b]{2}{*}{ TOTAL } & \\
\hline & & $16-29$ & $30-39$ & $40-49$ & $50+$ & & \\
\hline -Disorders of Peripheral NS & $350-359$ & 0 & 0 & 2 & 10 & 12 & 23 \\
\hline -Disorders of Eye & $360-379$ & 0 & 3 & 1 & 16 & 20 & 26 \\
\hline CIRCULATORY SYSTEM & $390-459$ & 2 & 4 & 8 & 68 & 82 & 105 \\
\hline -Hypertensive Dis & $401-405$ & 0 & 1 & 0 & 15 & 16 & 20 \\
\hline -Ischemic Heart Dis & $410-414$ & 0 & 0 & 1 & 23 & 24 & 29 \\
\hline -Dis of Pulmonary Circulation & $415-417$ & 0 & 0 & 0 & 0 & 0 & 1 \\
\hline -Dis of Veins, Lymphatics, Other & 451-459 & 1 & 1 & 3 & 14 & 19 & 26 \\
\hline RESPIRATORY SYSTEM & $460-519$ & 3 & 11 & 23 & 71 & 108 & 208 \\
\hline -Acute Respiratory Infections & $460-466$ & 0 & 3 & 6 & 23 & 32 & 70 \\
\hline -Other Dis Upper Respiratory Tract & $470-478$ & 1 & 4 & 7 & 25 & 37 & 57 \\
\hline -Pneumonia \& Influenza & $480-488$ & 1 & 2 & 3 & 12 & 18 & 26 \\
\hline -Chronic Obstructive Dis & $490-496$ & 1 & 2 & 4 & 11 & 18 & 46 \\
\hline -Other Respiratory Dis & $510-519$ & 0 & 0 & 3 & 0 & 3 & 9 \\
\hline DIGESTIVE SYSTEM & $520-579$ & 2 & 13 & 13 & 49 & 77 & 127 \\
\hline -Other Intestinal Dis & $560-569$ & 0 & 3 & 2 & 11 & 16 & 25 \\
\hline -Other Digestive Dis & $570-579$ & 0 & 5 & 3 & 9 & 17 & 33 \\
\hline GENITOURINARY SYSTEM & $580-629$ & 1 & 0 & 2 & 19 & 22 & 69 \\
\hline -Nephritis, Nephrosis & $580-589$ & 0 & 0 & 0 & 2 & 2 & 5 \\
\hline -Other Urinary Dis & $590-599$ & 1 & 0 & 2 & 7 & 10 & 16 \\
\hline -Male Genital Organ Dis & $600-608$ & 0 & 0 & 0 & 10 & 10 & 10 \\
\hline -Breast Disorders & $610-612$ & 0 & 0 & 0 & 0 & 0 & 4 \\
\hline -Other Female Disorders & $617-629$ & 0 & 0 & 0 & 0 & 0 & 34 \\
\hline SKIN AND SUBCUTANEOUS TISSUE & 680-709 & 2 & 3 & 4 & 15 & 24 & 28 \\
\hline -Infections & $680-686$ & 2 & 2 & 3 & 8 & 15 & 16 \\
\hline -Other Inflammatory Conditions & $690-698$ & 0 & 0 & 0 & 1 & 1 & 2 \\
\hline -Other & 700-709 & 0 & 1 & 1 & 6 & 8 & 10 \\
\hline $\begin{array}{l}\text { MUSCULOSKELETAL \& CONNECTIVE } \\
\text { TISSUE }\end{array}$ & 710-739 & 8 & 36 & 43 & 161 & 248 & 369 \\
\hline
\end{tabular}

(Continued)

*Only those diagnostic categories and gender/age combinations with at least one occurrence appear in this table. 


\section{Y-12 National Security Complex 2010}

\section{Absence Data}

Appendix G. Number of Diagnoses in Each Diagnostic Category by Gender and Age*

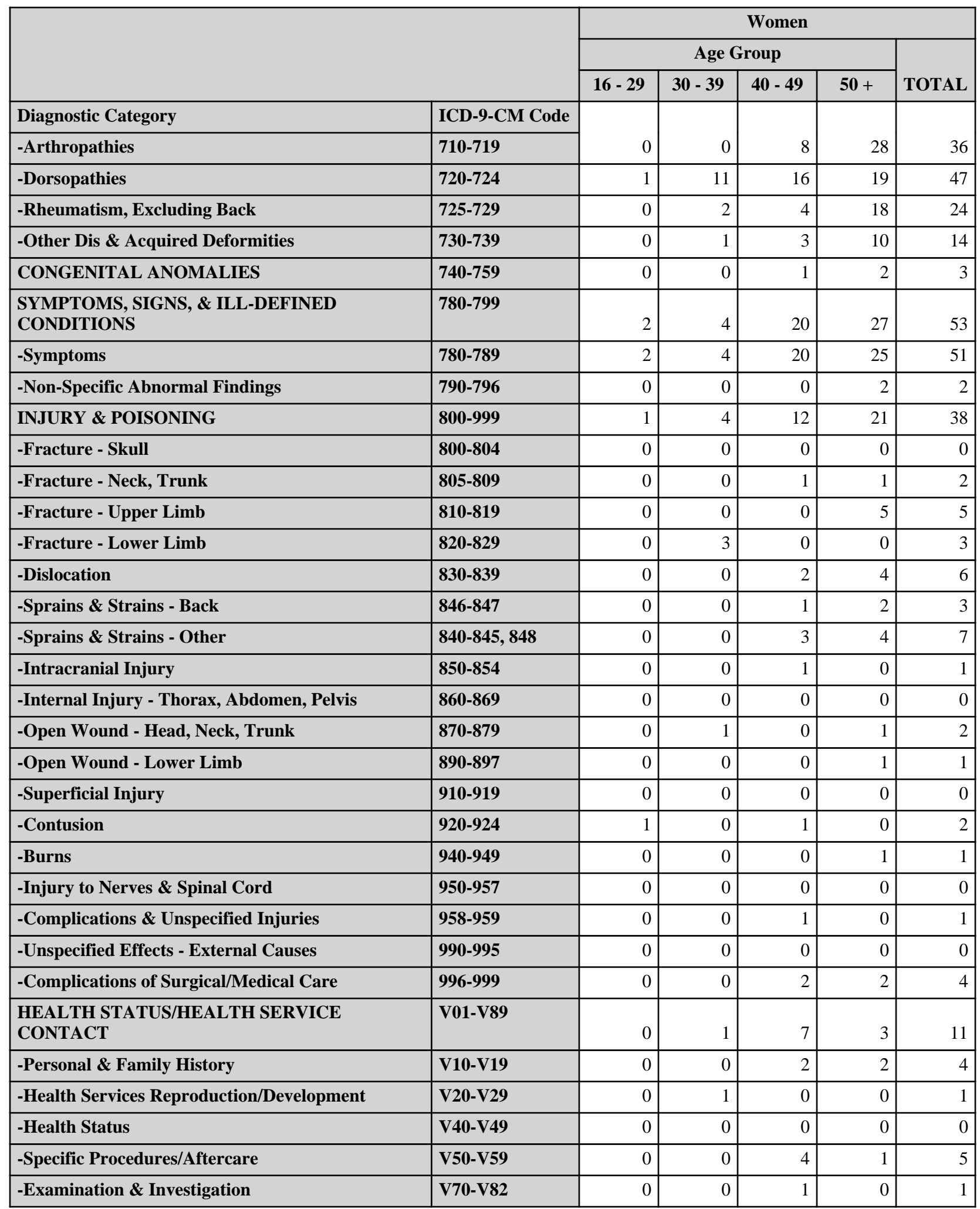

*Only those diagnostic categories and gender/age combinations with at least one occurrence appear in this table. 


\section{Y-12 National Security Complex 2010}

\section{Absence Data}

Appendix G. Number of Diagnoses in Each Diagnostic Category by Gender and Age*

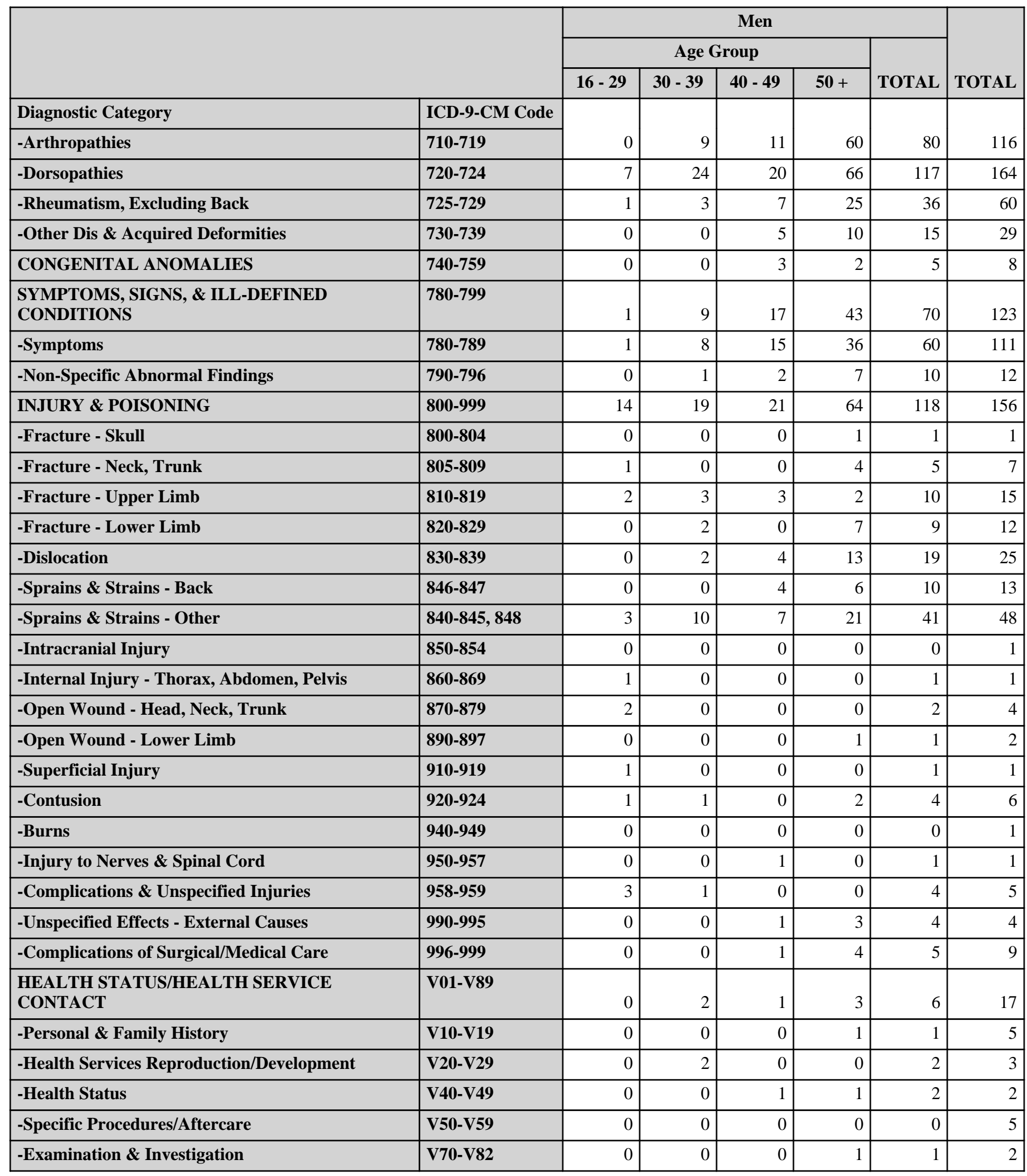

*Only those diagnostic categories and gender/age combinations with at least one occurrence appear in this table. 
Y-12 National Security Complex 2010

Absence Data

Appendix G. Number of Diagnoses in Each Diagnostic Category by Gender and Age*

\begin{tabular}{|c|c|c|c|c|c|c|c|c|c|c|c|}
\hline & \multicolumn{5}{|c|}{ Women } & \multicolumn{5}{|c|}{ Men } & \multirow[b]{3}{*}{ TOTAL } \\
\hline & \multicolumn{4}{|c|}{ Age Group } & \multirow[b]{2}{*}{ TOTAL } & \multicolumn{4}{|c|}{ Age Group } & \multirow[b]{2}{*}{ TOTAL } & \\
\hline & $16-29$ & $30-39$ & $40-49$ & $50+$ & & $16-29$ & 30 - 39 & $40-49$ & $50+$ & & \\
\hline Diagnostic Category & \multirow[b]{2}{*}{19} & \multirow[b]{2}{*}{56} & \multirow[b]{2}{*}{151} & \multirow[b]{2}{*}{312} & \multirow[b]{2}{*}{538} & \multirow[b]{2}{*}{43} & \multirow[b]{2}{*}{119} & \multirow[b]{2}{*}{158} & \multirow[b]{2}{*}{585} & \multirow[b]{2}{*}{905} & \multirow[b]{2}{*}{1,443} \\
\hline Total & & & & & & & & & & & \\
\hline
\end{tabular}

*Only those diagnostic categories and gender/age combinations with at least one occurrence appear in this table. 
Y-12 National Security Complex 2010

Absence Data

Appendix H. Total Number of Calendar Days Absent in Each Diagnostic Category by Gender and Age*

\begin{tabular}{|c|c|c|c|c|c|c|}
\hline & & \multicolumn{5}{|c|}{ Women } \\
\hline & & \multicolumn{4}{|c|}{ Age Group } & \multirow[b]{2}{*}{ TOTAL } \\
\hline & & $16-29$ & $30-39$ & $40-49$ & $50+$ & \\
\hline Diagnostic Category & ICD-9-CM Code & \multirow[b]{2}{*}{0} & \multirow[b]{2}{*}{7} & \multirow[b]{2}{*}{120} & \multirow[b]{2}{*}{173} & \multirow[b]{2}{*}{300} \\
\hline INFECTIOUS \& PARASITIC DISEASES (DIS) & 001-139 & & & & & \\
\hline MALIGNANT NEOPLASMS & $\begin{array}{l}140-208, \\
209.0-209.3, \\
230-234\end{array}$ & 0 & 0 & 195 & 415 & 610 \\
\hline BENIGN \& UNCERTAIN NEOPLASMS & $\begin{array}{l}209.4-209.6 \\
210-229,235-239\end{array}$ & 35 & 42 & 107 & 66 & 250 \\
\hline ENDOCRINE/METABOLIC/IMMUNITY & $240-279$ & 0 & 7 & 7 & 270 & 284 \\
\hline BLOOD \& BLOOD-FORMING ORGANS & $280-289$ & 0 & 0 & 0 & 215 & 215 \\
\hline MENTAL DISORDERS & 290-319 & 93 & 48 & 144 & 185 & 470 \\
\hline NERVOUS SYSTEM (NS) \& SENSE ORGANS & 320-389 & 0 & 71 & 331 & 999 & 1,401 \\
\hline CIRCULATORY SYSTEM & $390-459$ & 19 & 0 & 148 & 1,196 & 1,363 \\
\hline RESPIRATORY SYSTEM & $460-519$ & 110 & 148 & 234 & 798 & 1,290 \\
\hline DIGESTIVE SYSTEM & $520-579$ & 14 & 89 & 264 & 857 & 1,224 \\
\hline GENITOURINARY SYSTEM & $580-629$ & 89 & 195 & 486 & 678 & 1,448 \\
\hline SKIN AND SUBCUTANEOUS TISSUE & $680-709$ & 0 & 0 & 21 & 8 & 29 \\
\hline $\begin{array}{l}\text { MUSCULOSKELETAL \& CONNECTIVE } \\
\text { TISSUE }\end{array}$ & 710-739 & 89 & 509 & 869 & 4,085 & 5,552 \\
\hline CONGENITAL ANOMALIES & $740-759$ & 0 & 0 & 10 & 87 & 97 \\
\hline $\begin{array}{l}\text { SYMPTOMS, SIGNS, \& ILL-DEFINED } \\
\text { CONDITIONS }\end{array}$ & \begin{tabular}{|l|}
$780-799$ \\
\end{tabular} & 98 & 127 & 540 & 698 & 1,463 \\
\hline INJURY \& POISONING & $800-999$ & 28 & 143 & 482 & 1,165 & 1,818 \\
\hline
\end{tabular}

\footnotetext{
*Absences with >1 ICD-9-CM code in the same diagnostic category were counted only once. Only those diagnostic categories and gender/age combinations with at least one occurrence appear in this table.
} 
Y-12 National Security Complex 2010

Absence Data

Appendix H. Total Number of Calendar Days Absent in Each Diagnostic Category by Gender and Age*

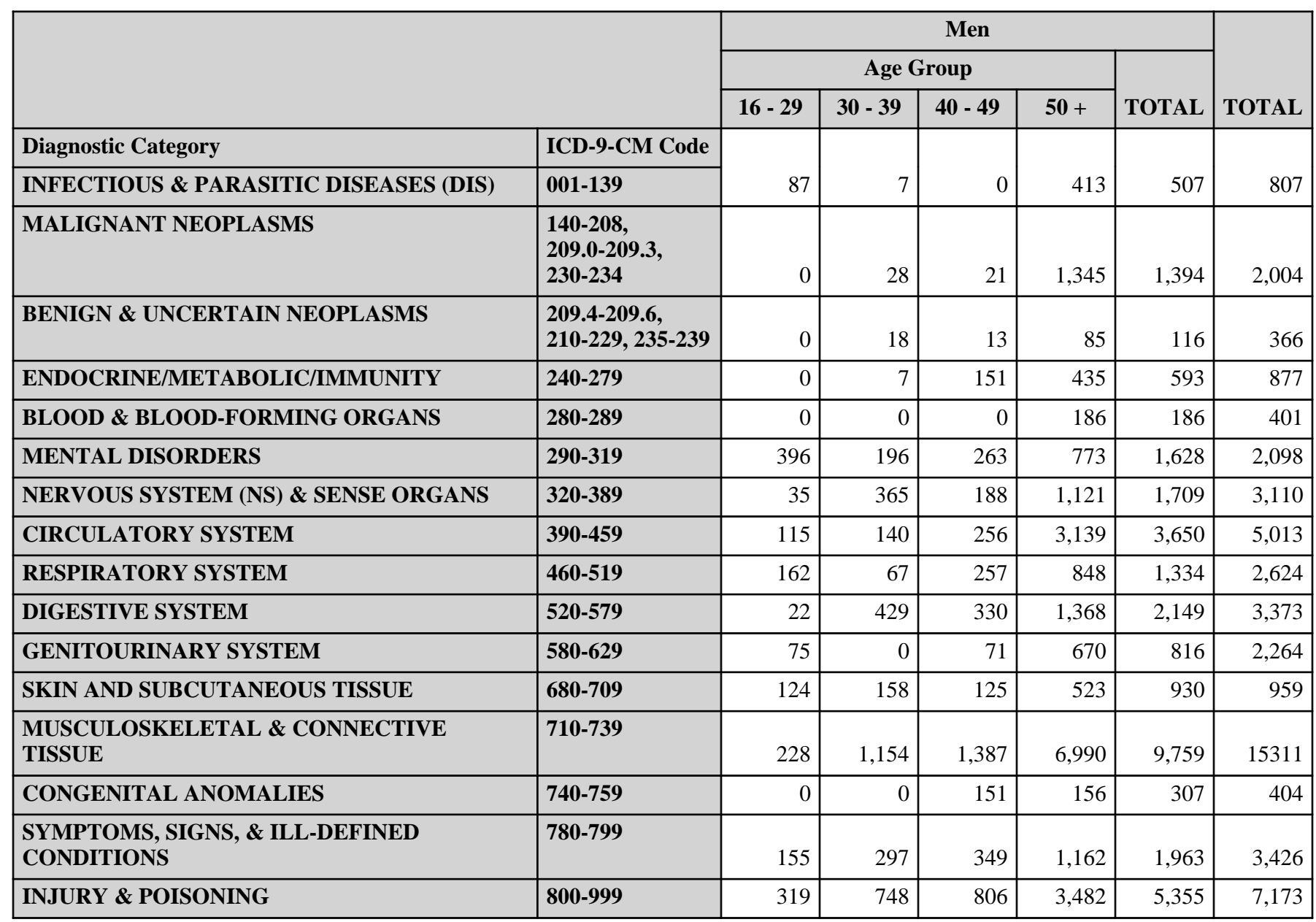

\footnotetext{
*Absences with >1 ICD-9-CM code in the same diagnostic category were counted only once. Only those diagnostic categories and gender/age combinations with at least one occurrence appear in this table.
} 


\section{Y-12 National Security Complex 2010}

\section{Absence Data}

Appendix I. Number of Diagnoses in Each Diagnostic Category by Gender and Job Category*

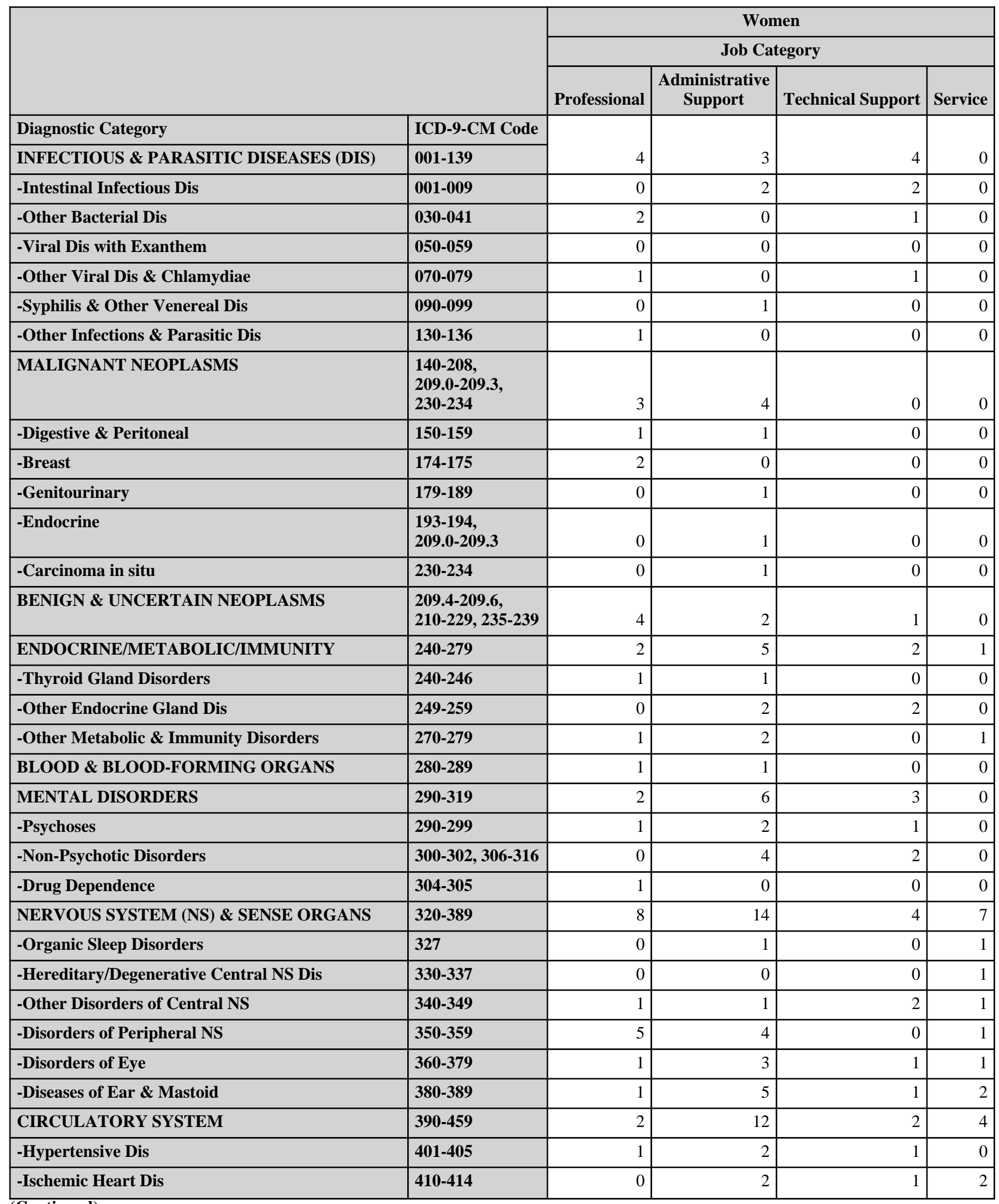

(Continued)

*Only those diagnostic categories and gender/job category combinations with at least one occurrence appear in this table. 


\section{Y-12 National Security Complex 2010}

Absence Data

Appendix I. Number of Diagnoses in Each Diagnostic Category by Gender and Job Category*

\begin{tabular}{|c|c|c|c|c|c|}
\hline & & \multicolumn{4}{|c|}{ Women } \\
\hline & & \multicolumn{3}{|c|}{ Job Category } & \multirow[b]{2}{*}{ TOTAL } \\
\hline & & Security and Fire & Crafts & Line Operators & \\
\hline Diagnostic Category & ICD-9-CM Code & \multirow[b]{2}{*}{0} & \multirow[b]{2}{*}{0} & \multirow[b]{2}{*}{1} & \multirow[b]{2}{*}{12} \\
\hline INFECTIOUS \& PARASITIC DISEASES (DIS) & 001-139 & & & & \\
\hline -Intestinal Infectious Dis & 001-009 & 0 & 0 & 0 & 4 \\
\hline -Other Bacterial Dis & 030-041 & 0 & 0 & 0 & 3 \\
\hline -Viral Dis with Exanthem & 050-059 & 0 & 0 & 1 & 1 \\
\hline -Other Viral Dis \& Chlamydiae & 070-079 & 0 & 0 & 0 & 2 \\
\hline -Syphilis \& Other Venereal Dis & 090-099 & 0 & 0 & 0 & 1 \\
\hline -Other Infections \& Parasitic Dis & $130-136$ & 0 & 0 & 0 & 1 \\
\hline MALIGNANT NEOPLASMS & $\begin{array}{l}140-208, \\
209.0-209.3 \\
230-234\end{array}$ & 0 & 1 & 0 & 8 \\
\hline -Digestive \& Peritoneal & $150-159$ & 0 & 0 & 0 & 2 \\
\hline -Breast & $174-175$ & 0 & 1 & 0 & 3 \\
\hline -Genitourinary & 179-189 & 0 & 0 & 0 & 1 \\
\hline -Endocrine & $\begin{array}{l}\text { 193-194, } \\
\text { 209.0-209.3 }\end{array}$ & 0 & 0 & 0 & 1 \\
\hline -Carcinoma in situ & $230-234$ & 0 & 0 & 0 & 1 \\
\hline BENIGN \& UNCERTAIN NEOPLASMS & $\begin{array}{l}209.4-209.6 \\
210-229,235-239\end{array}$ & 0 & 0 & 0 & 7 \\
\hline ENDOCRINE/METABOLIC/IMMUNITY & 240-279 & 0 & 0 & 0 & 10 \\
\hline -Thyroid Gland Disorders & $240-246$ & 0 & 0 & 0 & 2 \\
\hline -Other Endocrine Gland Dis & $249-259$ & 0 & 0 & 0 & 4 \\
\hline -Other Metabolic \& Immunity Disorders & 270-279 & 0 & 0 & 0 & 4 \\
\hline BLOOD \& BLOOD-FORMING ORGANS & $280-289$ & 0 & 0 & 0 & 2 \\
\hline MENTAL DISORDERS & $290-319$ & 0 & 0 & 1 & 12 \\
\hline -Psychoses & 290-299 & 0 & 0 & 0 & 4 \\
\hline -Non-Psychotic Disorders & $300-302,306-316$ & 0 & 0 & 1 & 7 \\
\hline -Drug Dependence & 304-305 & 0 & 0 & 0 & 1 \\
\hline NERVOUS SYSTEM (NS) \& SENSE ORGANS & $320-389$ & 0 & 3 & 1 & 37 \\
\hline -Organic Sleep Disorders & 327 & 0 & 0 & 0 & 2 \\
\hline -Hereditary/Degenerative Central NS Dis & 330-337 & 0 & 0 & 0 & 1 \\
\hline -Other Disorders of Central NS & 340-349 & 0 & 3 & 0 & 8 \\
\hline -Disorders of Peripheral NS & $350-359$ & 0 & 0 & 1 & 11 \\
\hline -Disorders of Eye & $360-379$ & 0 & 0 & 0 & 6 \\
\hline -Diseases of Ear \& Mastoid & $380-389$ & 0 & 0 & 0 & 9 \\
\hline CIRCULATORY SYSTEM & $390-459$ & 0 & 1 & 2 & 23 \\
\hline -Hypertensive Dis & $401-405$ & 0 & 0 & 0 & 4 \\
\hline -Ischemic Heart Dis & $410-414$ & 0 & 0 & 0 & 5 \\
\hline
\end{tabular}

(Continued)

*Only those diagnostic categories and gender/job category combinations with at least one occurrence appear in this table. 


\section{Y-12 National Security Complex 2010}

\section{Absence Data}

Appendix I. Number of Diagnoses in Each Diagnostic Category by Gender and Job Category*

\begin{tabular}{|c|c|c|c|c|c|}
\hline & \multicolumn{4}{|c|}{ Women } \\
\hline & & \multicolumn{4}{|c|}{ Job Category } \\
\hline & & Professional & $\begin{array}{c}\text { Administrative } \\
\text { Support }\end{array}$ & Technical Support & Service \\
\hline Diagnostic Category & ICD-9-CM Code & \multirow[b]{2}{*}{0} & \multirow[b]{2}{*}{1} & \multirow[b]{2}{*}{0} & \multirow[b]{2}{*}{0} \\
\hline -Dis of Pulmonary Circulation & $415-417$ & & & & \\
\hline -Other Heart Dis & $420-429$ & 0 & 1 & 0 & 0 \\
\hline -Cerebrovascular Dis & 430-438 & 0 & 1 & 0 & 1 \\
\hline -Dis of Arteries \& Capillaries & $440-449$ & 0 & 0 & 0 & 1 \\
\hline -Dis of Veins, Lymphatics, Other & 451-459 & 1 & 5 & 0 & 0 \\
\hline RESPIRATORY SYSTEM & $460-519$ & 13 & 43 & 16 & 18 \\
\hline -Acute Respiratory Infections & $460-466$ & 6 & 16 & 9 & 4 \\
\hline -Other Dis Upper Respiratory Tract & $470-478$ & 1 & 13 & 2 & 2 \\
\hline -Pneumonia \& Influenza & $480-488$ & 1 & 2 & 1 & 2 \\
\hline -Chronic Obstructive Dis & $490-496$ & 5 & 9 & 3 & 9 \\
\hline -Other Respiratory Dis & 510-519 & 0 & 3 & 1 & 1 \\
\hline DIGESTIVE SYSTEM & 520-579 & 13 & 23 & 5 & 4 \\
\hline -Oral Cavity, Saliva Glands, Jaw & 520-529 & 4 & 4 & 0 & 0 \\
\hline -Esophagus, Stomach, Duodenum & 530-538 & 1 & 2 & 0 & 3 \\
\hline -Appendicitis & $540-543$ & 0 & 1 & 0 & 0 \\
\hline -Hernias & $550-553$ & 1 & 0 & 0 & 1 \\
\hline -Enteritis, Colitis & 555-558 & 0 & 5 & 1 & 0 \\
\hline -Other Intestinal Dis & $560-569$ & 1 & 6 & 2 & 0 \\
\hline -Other Digestive Dis & 570-579 & 6 & 5 & 2 & 0 \\
\hline GENITOURINARY SYSTEM & 580-629 & 11 & 16 & 4 & 8 \\
\hline -Nephritis, Nephrosis & 580-589 & 0 & 1 & 0 & 2 \\
\hline -Other Urinary Dis & 590-599 & 2 & 0 & 0 & 1 \\
\hline -Breast Disorders & 610-612 & 1 & 1 & 0 & 1 \\
\hline -Other Female Disorders & 617-629 & 8 & 14 & 4 & 4 \\
\hline SKIN AND SUBCUTANEOUS TISSUE & 680-709 & 1 & 3 & 0 & 0 \\
\hline -Infections & 680-686 & 1 & 0 & 0 & 0 \\
\hline -Other Inflammatory Conditions & 690-698 & 0 & 1 & 0 & 0 \\
\hline -Other & 700-709 & 0 & 2 & 0 & 0 \\
\hline $\begin{array}{l}\text { MUSCULOSKELETAL \& CONNECTIVE } \\
\text { TISSUE }\end{array}$ & 710-739 & 29 & 41 & 11 & 16 \\
\hline -Arthropathies & 710-719 & 8 & 12 & 4 & 6 \\
\hline -Dorsopathies & 720-724 & 12 & 16 & 5 & 3 \\
\hline -Rheumatism, Excluding Back & $725-729$ & 3 & 7 & 2 & 5 \\
\hline -Other Dis \& Acquired Deformities & 730-739 & 6 & 6 & 0 & 2 \\
\hline CONGENITAL ANOMALIES & 740-759 & 1 & 2 & 0 & 0 \\
\hline $\begin{array}{l}\text { SYMPTOMS, SIGNS, \& ILL-DEFINED } \\
\text { CONDITIONS }\end{array}$ & 780-799 & 13 & 19 & 5 & 11 \\
\hline
\end{tabular}

(Continued)

*Only those diagnostic categories and gender/job category combinations with at least one occurrence appear in this table. 


\section{Y-12 National Security Complex 2010}

\section{Absence Data}

Appendix I. Number of Diagnoses in Each Diagnostic Category by Gender and Job Category*

\begin{tabular}{|c|c|c|c|c|c|}
\hline & & \multicolumn{4}{|c|}{ Women } \\
\hline & & \multicolumn{3}{|c|}{ Job Category } & \multirow[b]{2}{*}{ TOTAL } \\
\hline & & Security and Fire & Crafts & Line Operators & \\
\hline Diagnostic Category & ICD-9-CM Code & \multirow[b]{2}{*}{0} & \multirow[b]{2}{*}{0} & \multirow[b]{2}{*}{0} & \multirow[b]{2}{*}{1} \\
\hline -Dis of Pulmonary Circulation & $415-417$ & & & & \\
\hline -Other Heart Dis & $420-429$ & 0 & 0 & 0 & 1 \\
\hline -Cerebrovascular Dis & $430-438$ & 0 & 0 & 1 & 3 \\
\hline -Dis of Arteries \& Capillaries & $440-449$ & 0 & 0 & 1 & 2 \\
\hline -Dis of Veins, Lymphatics, Other & 451-459 & 0 & 1 & 0 & 7 \\
\hline RESPIRATORY SYSTEM & $460-519$ & 0 & 4 & 6 & 100 \\
\hline -Acute Respiratory Infections & $460-466$ & 0 & 1 & 2 & 38 \\
\hline -Other Dis Upper Respiratory Tract & $470-478$ & 0 & 0 & 2 & 20 \\
\hline -Pneumonia \& Influenza & $480-488$ & 0 & 1 & 1 & 8 \\
\hline -Chronic Obstructive Dis & $490-496$ & 0 & 1 & 1 & 28 \\
\hline -Other Respiratory Dis & $510-519$ & 0 & 1 & 0 & 6 \\
\hline DIGESTIVE SYSTEM & $520-579$ & 2 & 2 & 1 & 50 \\
\hline -Oral Cavity, Saliva Glands, Jaw & $520-529$ & 0 & 0 & 1 & 9 \\
\hline -Esophagus, Stomach, Duodenum & $530-538$ & 0 & 0 & 0 & 6 \\
\hline -Appendicitis & $540-543$ & 0 & 1 & 0 & 2 \\
\hline -Hernias & $550-553$ & 0 & 0 & 0 & 2 \\
\hline -Enteritis, Colitis & $555-558$ & 0 & 0 & 0 & 6 \\
\hline -Other Intestinal Dis & $560-569$ & 0 & 0 & 0 & 9 \\
\hline -Other Digestive Dis & $570-579$ & 2 & 1 & 0 & 16 \\
\hline GENITOURINARY SYSTEM & $580-629$ & 4 & 2 & 2 & 47 \\
\hline -Nephritis, Nephrosis & $580-589$ & 0 & 0 & 0 & 3 \\
\hline -Other Urinary Dis & $590-599$ & 2 & 0 & 1 & 6 \\
\hline -Breast Disorders & $610-612$ & 0 & 1 & 0 & 4 \\
\hline -Other Female Disorders & $617-629$ & 2 & 1 & 1 & 34 \\
\hline SKIN AND SUBCUTANEOUS TISSUE & $680-709$ & 0 & 0 & 0 & 4 \\
\hline -Infections & $680-686$ & 0 & 0 & 0 & 1 \\
\hline -Other Inflammatory Conditions & 690-698 & 0 & 0 & 0 & 1 \\
\hline -Other & 700-709 & 0 & 0 & 0 & 2 \\
\hline $\begin{array}{l}\text { MUSCULOSKELETAL \& CONNECTIVE } \\
\text { TISSUE }\end{array}$ & $710-739$ & 1 & 5 & 18 & 121 \\
\hline -Arthropathies & 710-719 & 0 & 1 & 5 & 36 \\
\hline -Dorsopathies & $720-724$ & 1 & 3 & 7 & 47 \\
\hline -Rheumatism, Excluding Back & 725-729 & 0 & 1 & 6 & 24 \\
\hline -Other Dis \& Acquired Deformities & 730-739 & 0 & 0 & 0 & 14 \\
\hline CONGENITAL ANOMALIES & $740-759$ & 0 & 0 & 0 & 3 \\
\hline $\begin{array}{l}\text { SYMPTOMS, SIGNS, \& ILL-DEFINED } \\
\text { CONDITIONS }\end{array}$ & 780-799 & 0 & 1 & 4 & 53 \\
\hline
\end{tabular}

(Continued)

*Only those diagnostic categories and gender/job category combinations with at least one occurrence appear in this table. 


\section{Y-12 National Security Complex 2010}

\section{Absence Data}

Appendix I. Number of Diagnoses in Each Diagnostic Category by Gender and Job Category*

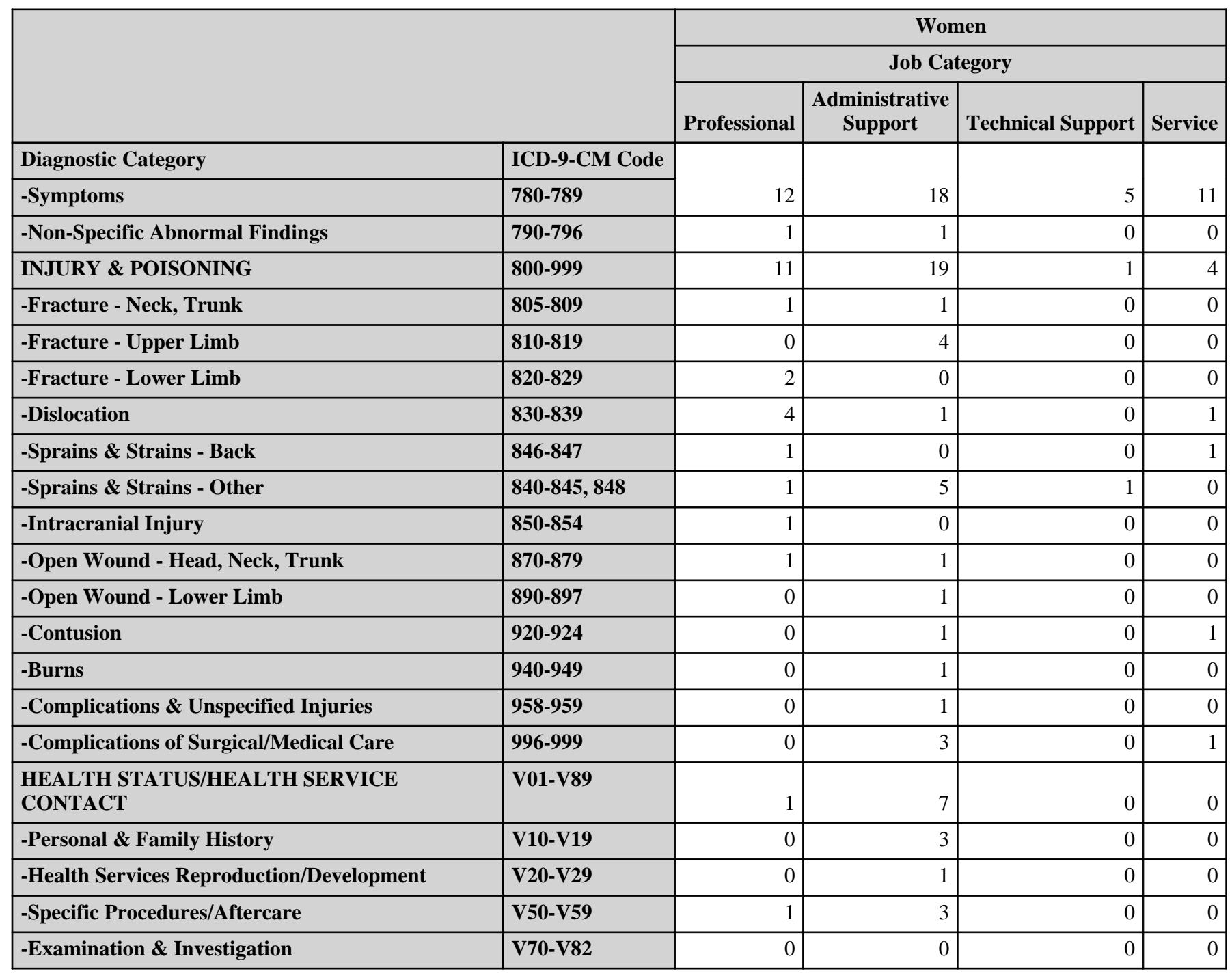

*Only those diagnostic categories and gender/job category combinations with at least one occurrence appear in this table. 


\section{Y-12 National Security Complex 2010}

\section{Absence Data}

Appendix I. Number of Diagnoses in Each Diagnostic Category by Gender and Job Category*

\begin{tabular}{|c|c|c|c|c|c|}
\hline & & \multicolumn{4}{|c|}{ Women } \\
\hline & & \multicolumn{3}{|c|}{ Job Category } & \multirow[b]{2}{*}{ TOTAL } \\
\hline & & Security and Fire & Crafts & Line Operators & \\
\hline Diagnostic Category & ICD-9-CM Code & \multirow[b]{2}{*}{0} & \multirow[b]{2}{*}{1} & \multirow[b]{2}{*}{4} & \multirow[b]{2}{*}{51} \\
\hline -Symptoms & $780-789$ & & & & \\
\hline -Non-Specific Abnormal Findings & $790-796$ & 0 & 0 & 0 & 2 \\
\hline INJURY \& POISONING & $800-999$ & 0 & 1 & 2 & 38 \\
\hline -Fracture - Neck, Trunk & 805-809 & 0 & 0 & 0 & 2 \\
\hline -Fracture - Upper Limb & 810-819 & 0 & 1 & 0 & 5 \\
\hline -Fracture - Lower Limb & $820-829$ & 0 & 0 & 1 & 3 \\
\hline -Dislocation & $830-839$ & 0 & 0 & 0 & 6 \\
\hline -Sprains \& Strains - Back & 846-847 & 0 & 0 & 1 & 3 \\
\hline -Sprains \& Strains - Other & $840-845,848$ & 0 & 0 & 0 & 7 \\
\hline -Intracranial Injury & $850-854$ & 0 & 0 & 0 & 1 \\
\hline -Open Wound - Head, Neck, Trunk & $870-879$ & 0 & 0 & 0 & 2 \\
\hline -Open Wound - Lower Limb & 890-897 & 0 & 0 & 0 & 1 \\
\hline -Contusion & $920-924$ & 0 & 0 & 0 & 2 \\
\hline -Burns & 940-949 & 0 & 0 & 0 & 1 \\
\hline -Complications \& Unspecified Injuries & 958-959 & 0 & 0 & 0 & 1 \\
\hline -Complications of Surgical/Medical Care & 996-999 & 0 & 0 & 0 & 4 \\
\hline $\begin{array}{l}\text { HEALTH STATUS/HEALTH SERVICE } \\
\text { CONTACT }\end{array}$ & V01-V89 & 1 & 2 & 0 & 11 \\
\hline -Personal \& Family History & V10-V19 & 0 & 1 & 0 & 4 \\
\hline -Health Services Reproduction/Development & V20-V29 & 0 & 0 & 0 & 1 \\
\hline -Specific Procedures/Aftercare & V50-V59 & 1 & 0 & 0 & 5 \\
\hline -Examination \& Investigation & V70-V82 & 0 & 1 & 0 & 1 \\
\hline
\end{tabular}

\begin{tabular}{|c|c|c|c|c|c|c|c|c|}
\hline & \multicolumn{8}{|c|}{ Women } \\
\hline & \multicolumn{7}{|c|}{ Job Category } & \multirow[b]{2}{*}{ TOTAL } \\
\hline & Professional & $\begin{array}{l}\text { Administrative } \\
\text { Support }\end{array}$ & $\begin{array}{l}\text { Technical } \\
\text { Support }\end{array}$ & Service & $\begin{array}{l}\text { Security and } \\
\text { Fire }\end{array}$ & Crafts & $\begin{array}{c}\text { Line } \\
\text { Operators }\end{array}$ & \\
\hline Diagnostic Category & \multirow[b]{2}{*}{119} & \multirow[b]{2}{*}{220} & \multirow[b]{2}{*}{58} & \multirow[b]{2}{*}{73} & \multirow[b]{2}{*}{8} & \multirow[b]{2}{*}{22} & \multirow[b]{2}{*}{38} & \multirow[b]{2}{*}{538} \\
\hline Total & & & & & & & & \\
\hline
\end{tabular}

*Only those diagnostic categories and gender/job category combinations with at least one occurrence appear in this table. 


\section{Y-12 National Security Complex 2010}

\section{Absence Data}

Appendix I. Number of Diagnoses in Each Diagnostic Category by Gender and Job Category*

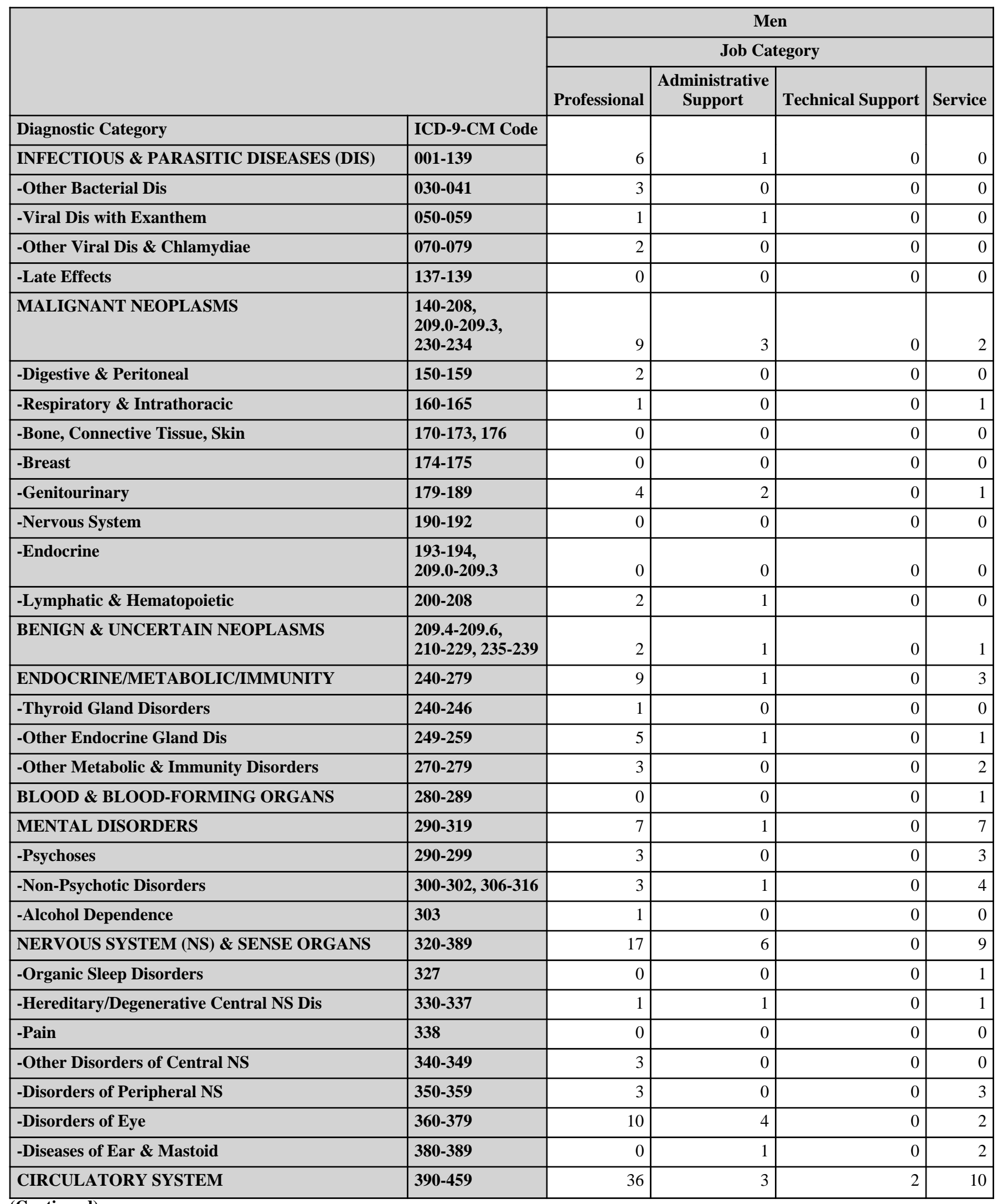

(Continued)

*Only those diagnostic categories and gender/job category combinations with at least one occurrence appear in this table. 


\section{Y-12 National Security Complex 2010}

\section{Absence Data}

Appendix I. Number of Diagnoses in Each Diagnostic Category by Gender and Job Category*

\begin{tabular}{|c|c|c|c|c|c|}
\hline & & \multicolumn{4}{|c|}{ Men } \\
\hline & & \multicolumn{3}{|c|}{ Job Category } & \multirow[b]{2}{*}{ TOTAL } \\
\hline & & Security and Fire & Crafts & Line Operators & \\
\hline Diagnostic Category & ICD-9-CM Code & \multirow[b]{2}{*}{0} & \multirow[b]{2}{*}{5} & \multirow[b]{2}{*}{0} & \multirow[b]{2}{*}{12} \\
\hline INFECTIOUS \& PARASITIC DISEASES (DIS) & 001-139 & & & & \\
\hline -Other Bacterial Dis & 030-041 & 0 & 2 & 0 & 5 \\
\hline -Viral Dis with Exanthem & 050-059 & 0 & 1 & 0 & 3 \\
\hline -Other Viral Dis \& Chlamydiae & 070-079 & 0 & 1 & 0 & 3 \\
\hline -Late Effects & $137-139$ & 0 & 1 & 0 & 1 \\
\hline MALIGNANT NEOPLASMS & $\begin{array}{l}140-208, \\
209.0-209.3 \\
230-234\end{array}$ & 1 & 7 & 0 & 22 \\
\hline -Digestive \& Peritoneal & $150-159$ & 0 & 0 & 0 & 2 \\
\hline -Respiratory \& Intrathoracic & $160-165$ & 0 & 2 & 0 & 4 \\
\hline -Bone, Connective Tissue, Skin & $170-173,176$ & 0 & 2 & 0 & 2 \\
\hline -Breast & $174-175$ & 0 & 1 & 0 & 1 \\
\hline -Genitourinary & 179-189 & 0 & 0 & 0 & 7 \\
\hline -Nervous System & 190-192 & 1 & 0 & 0 & 1 \\
\hline -Endocrine & $\begin{array}{l}193-194, \\
209.0-209.3\end{array}$ & 0 & 1 & 0 & 1 \\
\hline -Lymphatic \& Hematopoietic & $200-208$ & 0 & 1 & 0 & 4 \\
\hline BENIGN \& UNCERTAIN NEOPLASMS & $\begin{array}{l}209.4-209.6, \\
210-229,235-239\end{array}$ & 0 & 6 & 0 & 10 \\
\hline ENDOCRINE/METABOLIC/IMMUNITY & $240-279$ & 0 & 6 & 2 & 21 \\
\hline -Thyroid Gland Disorders & $240-246$ & 0 & 3 & 0 & 4 \\
\hline -Other Endocrine Gland Dis & 249-259 & 0 & 1 & 1 & 9 \\
\hline -Other Metabolic \& Immunity Disorders & $270-279$ & 0 & 2 & 1 & 8 \\
\hline BLOOD \& BLOOD-FORMING ORGANS & $280-289$ & 0 & 0 & 0 & 1 \\
\hline MENTAL DISORDERS & 290-319 & 0 & 12 & 2 & 29 \\
\hline -Psychoses & $290-299$ & 0 & 6 & 2 & 14 \\
\hline -Non-Psychotic Disorders & $300-302,306-316$ & 0 & 5 & 0 & 13 \\
\hline -Alcohol Dependence & 303 & 0 & 1 & 0 & 2 \\
\hline NERVOUS SYSTEM (NS) \& SENSE ORGANS & $320-389$ & 1 & 12 & 5 & 50 \\
\hline -Organic Sleep Disorders & 327 & 0 & 1 & 0 & 2 \\
\hline -Hereditary/Degenerative Central NS Dis & 330-337 & 0 & 0 & 0 & 3 \\
\hline -Pain & 338 & 0 & 2 & 0 & 2 \\
\hline -Other Disorders of Central NS & $340-349$ & 0 & 1 & 0 & 4 \\
\hline -Disorders of Peripheral NS & 350-359 & 1 & 3 & 2 & 12 \\
\hline -Disorders of Eye & $360-379$ & 0 & 2 & 2 & 20 \\
\hline -Diseases of Ear \& Mastoid & 380-389 & 0 & 3 & 1 & 7 \\
\hline CIRCULATORY SYSTEM & $390-459$ & 4 & 24 & 3 & 82 \\
\hline
\end{tabular}

(Continued)

*Only those diagnostic categories and gender/job category combinations with at least one occurrence appear in this table. 


\section{Y-12 National Security Complex 2010}

\section{Absence Data}

Appendix I. Number of Diagnoses in Each Diagnostic Category by Gender and Job Category*

\begin{tabular}{|c|c|c|c|c|c|}
\hline & \multicolumn{4}{|c|}{ Men } \\
\hline & & \multicolumn{4}{|c|}{ Job Category } \\
\hline & & Professional & $\begin{array}{c}\text { Administrative } \\
\text { Support }\end{array}$ & Technical Support & Service \\
\hline Diagnostic Category & ICD-9-CM Code & \multirow[b]{2}{*}{8} & \multirow[b]{2}{*}{0} & \multirow[b]{2}{*}{0} & \multirow[b]{2}{*}{2} \\
\hline -Hypertensive Dis & $401-405$ & & & & \\
\hline -Other Heart Dis & $420-429$ & 6 & 0 & 0 & 0 \\
\hline -Cerebrovascular Dis & $430-438$ & 4 & 1 & 0 & 1 \\
\hline -Dis of Arteries \& Capillaries & $440-449$ & 0 & 0 & 0 & 1 \\
\hline -Dis of Veins, Lymphatics, Other & $451-459$ & 5 & 0 & 2 & 1 \\
\hline -Pneumonia \& Influenza & $480-488$ & 7 & 0 & 0 & 1 \\
\hline -Chronic Obstructive Dis & $490-496$ & 6 & 3 & 0 & 4 \\
\hline -Other Respiratory Dis & $510-519$ & 1 & 0 & 0 & 0 \\
\hline DIGESTIVE SYSTEM & $520-579$ & 30 & 8 & 6 & 3 \\
\hline -Oral Cavity, Saliva Glands, Jaw & $520-529$ & 2 & 2 & 1 & 0 \\
\hline -Esophagus, Stomach, Duodenum & $530-538$ & 4 & 2 & 0 & 1 \\
\hline -Hernias & $550-553$ & 7 & 1 & 0 & 2 \\
\hline -Enteritis, Colitis & $555-558$ & 2 & 0 & 1 & 0 \\
\hline SKIN AND SUBCUTANEOUS TISSUE & $680-709$ & 8 & 1 & 2 & 2 \\
\hline -Infections & $680-686$ & 7 & 0 & 2 & 2 \\
\hline -Other Inflammatory Conditions & $690-698$ & 1 & 0 & 0 & 0 \\
\hline -Other & 700-709 & 0 & 1 & 0 & 0 \\
\hline $\begin{array}{l}\text { MUSCULOSKELETAL \& CONNECTIVE } \\
\text { TISSUE }\end{array}$ & $710-739$ & 94 & 12 & 8 & 27 \\
\hline -Arthropathies & $710-719$ & 35 & 6 & 4 & 5 \\
\hline -Dorsopathies & $720-724$ & 42 & 4 & 2 & 17 \\
\hline -Rheumatism, Excluding Back & $725-729$ & 13 & 2 & 2 & 5 \\
\hline -Other Dis \& Acquired Deformities & \begin{tabular}{|l|}
$730-739$ \\
\end{tabular} & 4 & 0 & 0 & 0 \\
\hline CONGENITAL ANOMALIES & 740-759 & 2 & 0 & 0 & 0 \\
\hline $\begin{array}{l}\text { SYMPTOMS, SIGNS, \& ILL-DEFINED } \\
\text { CONDITIONS }\end{array}$ & 780-799 & 24 & 8 & 1 & 8 \\
\hline -Symptoms & $780-789$ & 22 & 7 & 1 & 6 \\
\hline
\end{tabular}

(Continued)

*Only those diagnostic categories and gender/job category combinations with at least one occurrence appear in this table. 


\section{Y-12 National Security Complex 2010}

\section{Absence Data}

Appendix I. Number of Diagnoses in Each Diagnostic Category by Gender and Job Category*

\begin{tabular}{|c|c|c|c|c|c|}
\hline & & \multicolumn{4}{|c|}{ Men } \\
\hline & & \multicolumn{3}{|c|}{ Job Category } & \multirow[b]{2}{*}{ TOTAL } \\
\hline & & Security and Fire & Crafts & Line Operators & \\
\hline Diagnostic Category & ICD-9-CM Code & \multirow[b]{2}{*}{1} & \multirow[b]{2}{*}{3} & \multirow[b]{2}{*}{2} & \multirow[b]{2}{*}{16} \\
\hline -Hypertensive Dis & $401-405$ & & & & \\
\hline -Ischemic Heart Dis & $410-414$ & 1 & 3 & 0 & 24 \\
\hline -Other Heart Dis & $420-429$ & 1 & 3 & 0 & 10 \\
\hline -Cerebrovascular Dis & $430-438$ & 0 & 3 & 0 & 9 \\
\hline -Dis of Arteries \& Capillaries & $440-449$ & 1 & 1 & 1 & 4 \\
\hline -Dis of Veins, Lymphatics, Other & 451-459 & 0 & 11 & 0 & 19 \\
\hline RESPIRATORY SYSTEM & $460-519$ & 0 & 37 & 13 & 108 \\
\hline -Acute Respiratory Infections & $460-466$ & 0 & 12 & 6 & 32 \\
\hline -Other Dis Upper Respiratory Tract & $470-478$ & 0 & 10 & 5 & 37 \\
\hline -Pneumonia \& Influenza & $480-488$ & 0 & 8 & 2 & 18 \\
\hline -Chronic Obstructive Dis & $490-496$ & 0 & 5 & 0 & 18 \\
\hline -Other Respiratory Dis & $510-519$ & 0 & 2 & 0 & 3 \\
\hline DIGESTIVE SYSTEM & $520-579$ & 0 & 23 & 7 & 77 \\
\hline -Oral Cavity, Saliva Glands, Jaw & $520-529$ & 0 & 4 & 0 & 9 \\
\hline -Esophagus, Stomach, Duodenum & $530-538$ & 0 & 0 & 0 & 7 \\
\hline -Hernias & $550-553$ & 0 & 8 & 1 & 19 \\
\hline -Enteritis, Colitis & $555-558$ & 0 & 4 & 2 & 9 \\
\hline -Other Intestinal Dis & $560-569$ & 0 & 4 & 2 & 16 \\
\hline -Other Digestive Dis & $570-579$ & 0 & 3 & 2 & 17 \\
\hline GENITOURINARY SYSTEM & $580-629$ & 2 & 6 & 5 & 22 \\
\hline -Nephritis, Nephrosis & $580-589$ & 0 & 0 & 0 & 2 \\
\hline -Other Urinary Dis & $590-599$ & 1 & 1 & 4 & 10 \\
\hline -Male Genital Organ Dis & $600-608$ & 1 & 5 & 1 & 10 \\
\hline SKIN AND SUBCUTANEOUS TISSUE & $680-709$ & 0 & 9 & 2 & 24 \\
\hline -Infections & $680-686$ & 0 & 4 & 0 & 15 \\
\hline -Other Inflammatory Conditions & $690-698$ & 0 & 0 & 0 & 1 \\
\hline -Other & 700-709 & 0 & 5 & 2 & 8 \\
\hline $\begin{array}{l}\text { MUSCULOSKELETAL \& CONNECTIVE } \\
\text { TISSUE }\end{array}$ & $710-739$ & 10 & 64 & 33 & 248 \\
\hline -Arthropathies & $710-719$ & 2 & 19 & 9 & 80 \\
\hline -Dorsopathies & $720-724$ & 5 & 29 & 18 & 117 \\
\hline -Rheumatism, Excluding Back & $725-729$ & 1 & 8 & 5 & 36 \\
\hline -Other Dis \& Acquired Deformities & $730-739$ & 2 & 8 & 1 & 15 \\
\hline CONGENITAL ANOMALIES & 740-759 & 0 & 3 & 0 & 5 \\
\hline $\begin{array}{l}\text { SYMPTOMS, SIGNS, \& ILL-DEFINED } \\
\text { CONDITIONS }\end{array}$ & $780-799$ & 2 & 17 & 10 & 70 \\
\hline -Symptoms & $780-789$ & 2 & 13 & 9 & 60 \\
\hline
\end{tabular}

(Continued)

*Only those diagnostic categories and gender/job category combinations with at least one occurrence appear in this table. 


\section{Y-12 National Security Complex 2010}

\section{Absence Data}

Appendix I. Number of Diagnoses in Each Diagnostic Category by Gender and Job Category*

\begin{tabular}{|c|c|c|c|c|c|}
\hline & & \multicolumn{4}{|c|}{ Men } \\
\hline & & \multicolumn{4}{|c|}{ Job Category } \\
\hline & & Professional & $\begin{array}{c}\text { Administrative } \\
\text { Support }\end{array}$ & Technical Support & Service \\
\hline Diagnostic Category & ICD-9-CM Code & \multirow[b]{2}{*}{2} & \multirow[b]{2}{*}{1} & \multirow[b]{2}{*}{0} & \multirow[b]{2}{*}{2} \\
\hline -Non-Specific Abnormal Findings & $790-796$ & & & & \\
\hline -Fracture - Skull & \begin{tabular}{|l|}
$800-804$ \\
\end{tabular} & 0 & 0 & 0 & 0 \\
\hline -Fracture - Neck, Trunk & 805-809 & 0 & 0 & 0 & 2 \\
\hline -Fracture - Upper Limb & $810-819$ & 3 & 1 & 0 & 0 \\
\hline -Fracture - Lower Limb & $820-829$ & 3 & 0 & 1 & 0 \\
\hline -Internal Injury - Thorax, Abdomen, Pelvis & $860-869$ & 0 & 0 & 0 & 0 \\
\hline -Open Wound - Head, Neck, Trunk & 870-879 & 0 & 0 & 0 & 0 \\
\hline -Open Wound - Lower Limb & 890-897 & 1 & 0 & 0 & 0 \\
\hline -Superficial Injury & $910-919$ & 0 & 0 & 0 & 0 \\
\hline -Contusion & 920-924 & 3 & 0 & 0 & 0 \\
\hline -Injury to Nerves \& Spinal Cord & $950-957$ & 0 & 0 & 0 & 0 \\
\hline -Complications \& Unspecified Injuries & 958-959 & 0 & 0 & 0 & 2 \\
\hline -Unspecified Effects - External Causes & $990-995$ & 2 & 0 & 0 & 0 \\
\hline -Examination \& Investigation & V70-V82 & 1 & 0 & 0 & 0 \\
\hline
\end{tabular}

*Only those diagnostic categories and gender/job category combinations with at least one occurrence appear in this table. 


\section{Y-12 National Security Complex 2010}

\section{Absence Data}

Appendix I. Number of Diagnoses in Each Diagnostic Category by Gender and Job Category*

\begin{tabular}{|c|c|c|c|c|c|}
\hline & & \multicolumn{4}{|c|}{ Men } \\
\hline & & \multicolumn{3}{|c|}{ Job Category } & \multirow[b]{2}{*}{ TOTAL } \\
\hline & & Security and Fire & Crafts & Line Operators & \\
\hline Diagnostic Category & ICD-9-CM Code & \multirow[b]{2}{*}{0} & \multirow[b]{2}{*}{4} & \multirow[b]{2}{*}{1} & \multirow[b]{2}{*}{10} \\
\hline -Non-Specific Abnormal Findings & $790-796$ & & & & \\
\hline INJURY \& POISONING & $800-999$ & 1 & 41 & 14 & 118 \\
\hline -Fracture - Skull & $800-804$ & 0 & 1 & 0 & 1 \\
\hline -Fracture - Neck, Trunk & $805-809$ & 0 & 3 & 0 & 5 \\
\hline -Fracture - Upper Limb & $810-819$ & 0 & 4 & 2 & 10 \\
\hline -Fracture - Lower Limb & $820-829$ & 0 & 3 & 2 & 9 \\
\hline -Dislocation & $830-839$ & 0 & 6 & 3 & 19 \\
\hline -Sprains \& Strains - Back & 846-847 & 0 & 3 & 0 & 10 \\
\hline -Sprains \& Strains - Other & $840-845,848$ & 0 & 10 & 6 & 41 \\
\hline -Internal Injury - Thorax, Abdomen, Pelvis & $860-869$ & 0 & 1 & 0 & 1 \\
\hline -Open Wound - Head, Neck, Trunk & $870-879$ & 0 & 2 & 0 & 2 \\
\hline -Open Wound - Lower Limb & $890-897$ & 0 & 0 & 0 & 1 \\
\hline -Superficial Injury & 910-919 & 0 & 1 & 0 & 1 \\
\hline -Contusion & 920-924 & 0 & 1 & 0 & 4 \\
\hline -Injury to Nerves \& Spinal Cord & 950-957 & 0 & 1 & 0 & 1 \\
\hline -Complications \& Unspecified Injuries & 958-959 & 1 & 1 & 0 & 4 \\
\hline -Unspecified Effects - External Causes & $990-995$ & 0 & 2 & 0 & 4 \\
\hline -Complications of Surgical/Medical Care & 996-999 & 0 & 2 & 1 & 5 \\
\hline $\begin{array}{l}\text { HEALTH STATUS/HEALTH SERVICE } \\
\text { CONTACT }\end{array}$ & V01-V89 & 0 & 1 & 1 & 6 \\
\hline -Personal \& Family History & V10-V19 & 0 & 0 & 0 & 1 \\
\hline -Health Services Reproduction/Development & V20-V29 & 0 & 0 & 1 & 2 \\
\hline -Health Status & V40-V49 & 0 & 1 & 0 & 2 \\
\hline -Examination \& Investigation & V70-V82 & 0 & 0 & 0 & 1 \\
\hline
\end{tabular}

\begin{tabular}{|c|c|c|c|c|c|c|c|c|}
\hline & \multicolumn{8}{|c|}{ Men } \\
\hline & \multicolumn{7}{|c|}{ Job Category } & \multirow[b]{2}{*}{ TOTAL } \\
\hline & Professional & $\begin{array}{c}\text { Administrative } \\
\text { Support }\end{array}$ & $\begin{array}{l}\text { Technical } \\
\text { Support }\end{array}$ & Service & $\begin{array}{l}\text { Security and } \\
\text { Fire }\end{array}$ & Crafts & $\begin{array}{c}\text { Line } \\
\text { Operators }\end{array}$ & \\
\hline Diagnostic Category & \multirow[b]{2}{*}{336} & \multirow[b]{2}{*}{58} & \multirow[b]{2}{*}{27} & \multirow[b]{2}{*}{93} & \multirow[b]{2}{*}{21} & \multirow[b]{2}{*}{273} & \multirow[b]{2}{*}{97} & \multirow[b]{2}{*}{905} \\
\hline Total & & & & & & & & \\
\hline
\end{tabular}

*Only those diagnostic categories and gender/job category combinations with at least one occurrence appear in this table. 
Y-12 National Security Complex 2010

Absence Data

Appendix J. Total Number of Calendar Days Absent in Each Diagnostic Category by Gender and Job Category*

\begin{tabular}{|c|c|c|c|c|c|}
\hline & & \multicolumn{4}{|c|}{ Women } \\
\hline & & \multicolumn{4}{|c|}{ Job Category } \\
\hline & & Professional & $\begin{array}{l}\text { Administrative } \\
\text { Support }\end{array}$ & Technical Support & Service \\
\hline Diagnostic Category & ICD-9-CM Code & \multirow[b]{2}{*}{108} & \multirow[b]{2}{*}{26} & \multirow[b]{2}{*}{138} & \multirow[b]{2}{*}{0} \\
\hline INFECTIOUS \& PARASITIC DISEASES (DIS) & 001-139 & & & & \\
\hline BENIGN \& UNCERTAIN NEOPLASMS & $\begin{array}{l}209.4-209.6 \\
210-229,235-239\end{array}$ & 169 & 58 & 23 & 0 \\
\hline ENDOCRINE/METABOLIC/IMMUNITY & $240-279$ & 35 & 89 & 119 & 41 \\
\hline CIRCULATORY SYSTEM & $390-459$ & 150 & 694 & 42 & 176 \\
\hline RESPIRATORY SYSTEM & $460-519$ & 88 & 488 & 202 & 344 \\
\hline DIGESTIVE SYSTEM & $520-579$ & 393 & 475 & 70 & 163 \\
\hline GENITOURINARY SYSTEM & $580-629$ & 268 & 450 & 126 & 339 \\
\hline SKIN AND SUBCUTANEOUS TISSUE & 680-709 & 7 & 22 & 0 & 0 \\
\hline $\begin{array}{l}\text { MUSCULOSKELETAL \& CONNECTIVE } \\
\text { TISSUE }\end{array}$ & 710-739 & 1,061 & 2,180 & 349 & 734 \\
\hline CONGENITAL ANOMALIES & $740-759$ & 33 & 64 & 0 & 0 \\
\hline $\begin{array}{l}\text { SYMPTOMS, SIGNS, \& ILL-DEFINED } \\
\text { CONDITIONS }\end{array}$ & 780-799 & 348 & 515 & 58 & 303 \\
\hline
\end{tabular}

\footnotetext{
*Absences with >1 ICD-9-CM code in the same diagnostic category were counted only once. Only those diagnostic categories and gender/job category combinations with at least one occurrence appear in this table.
} 
Y-12 National Security Complex 2010

Absence Data

Appendix J. Total Number of Calendar Days Absent in Each Diagnostic Category by Gender and Job Category*

\begin{tabular}{|c|c|c|c|c|c|}
\hline & & \multicolumn{4}{|c|}{ Women } \\
\hline & & \multicolumn{3}{|c|}{ Job Category } & \multirow[b]{2}{*}{ TOTAL } \\
\hline & & Security and Fire & Crafts & Line Operators & \\
\hline Diagnostic Category & ICD-9-CM Code & \multirow[b]{2}{*}{0} & \multirow[b]{2}{*}{0} & \multirow[b]{2}{*}{28} & \multirow[b]{2}{*}{300} \\
\hline INFECTIOUS \& PARASITIC DISEASES (DIS) & 001-139 & & & & \\
\hline MALIGNANT NEOPLASMS & $\begin{array}{l}\text { 140-208, } \\
209.0-209.3 \\
230-234\end{array}$ & 0 & 140 & 0 & 610 \\
\hline BENIGN \& UNCERTAIN NEOPLASMS & $\begin{array}{l}209.4-209.6 \\
210-229,235-239\end{array}$ & 0 & 0 & 0 & 250 \\
\hline ENDOCRINE/METABOLIC/IMMUNITY & $240-279$ & 0 & 0 & 0 & 284 \\
\hline BLOOD \& BLOOD-FORMING ORGANS & $280-289$ & 0 & 0 & 0 & 215 \\
\hline MENTAL DISORDERS & 290-319 & 0 & 0 & 76 & 470 \\
\hline NERVOUS SYSTEM (NS) \& SENSE ORGANS & $320-389$ & 0 & 71 & 176 & 1,401 \\
\hline CIRCULATORY SYSTEM & $390-459$ & 0 & 19 & 282 & 1,363 \\
\hline RESPIRATORY SYSTEM & $460-519$ & 0 & 85 & 83 & 1,290 \\
\hline DIGESTIVE SYSTEM & $520-579$ & 16 & 90 & 17 & 1,224 \\
\hline GENITOURINARY SYSTEM & $580-629$ & 77 & 161 & 27 & 1,448 \\
\hline SKIN AND SUBCUTANEOUS TISSUE & 680-709 & 0 & 0 & 0 & 29 \\
\hline $\begin{array}{l}\text { MUSCULOSKELETAL \& CONNECTIVE } \\
\text { TISSUE }\end{array}$ & 710-739 & 7 & 285 & 936 & 5,552 \\
\hline CONGENITAL ANOMALIES & $740-759$ & 0 & 0 & 0 & 97 \\
\hline $\begin{array}{l}\text { SYMPTOMS, SIGNS, \& ILL-DEFINED } \\
\text { CONDITIONS }\end{array}$ & 780-799 & 0 & 184 & 55 & 1,463 \\
\hline INJURY \& POISONING & $800-999$ & 0 & 138 & 130 & 1,818 \\
\hline
\end{tabular}

\footnotetext{
*Absences with >1 ICD-9-CM code in the same diagnostic category were counted only once. Only those diagnostic categories and gender/job category combinations with at least one occurrence appear in this table.
} 
Y-12 National Security Complex 2010

Absence Data

Appendix J. Total Number of Calendar Days Absent in Each Diagnostic Category by Gender and Job Category*

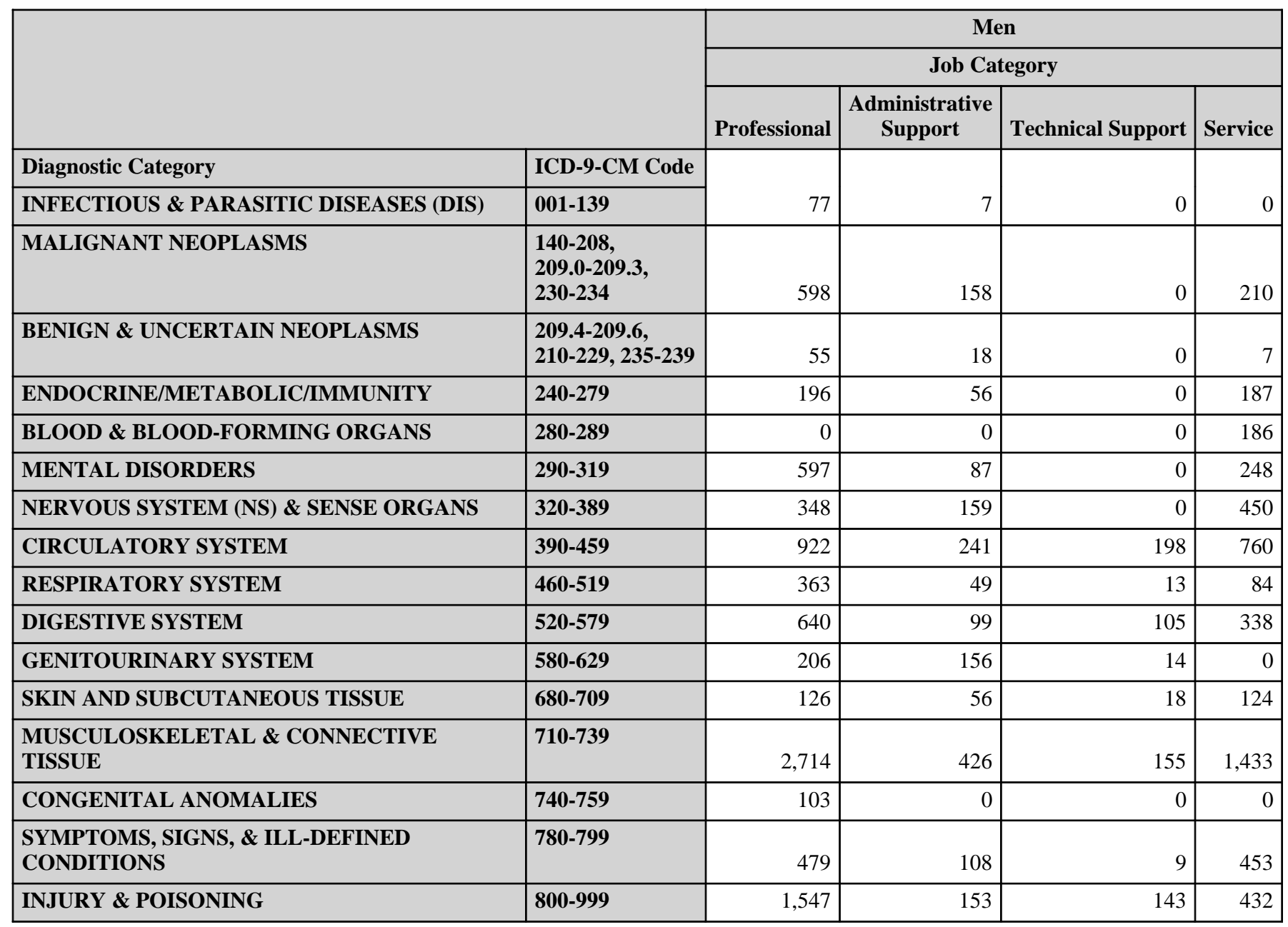

\footnotetext{
*Absences with >1 ICD-9-CM code in the same diagnostic category were counted only once. Only those diagnostic categories and gender/job category combinations with at least one occurrence appear in this table.
} 
Y-12 National Security Complex 2010

Absence Data

Appendix J. Total Number of Calendar Days Absent in Each Diagnostic Category by Gender and Job Category*

\begin{tabular}{|c|c|c|c|c|c|}
\hline & & \multicolumn{4}{|c|}{ Men } \\
\hline & & \multicolumn{3}{|c|}{ Job Category } & \multirow[b]{2}{*}{ TOTAL } \\
\hline & & Security and Fire & Crafts & Line Operators & \\
\hline Diagnostic Category & ICD-9-CM Code & \multirow[b]{2}{*}{0} & \multirow[b]{2}{*}{423} & \multirow[b]{2}{*}{0} & \multirow[b]{2}{*}{507} \\
\hline INFECTIOUS \& PARASITIC DISEASES (DIS) & 001-139 & & & & \\
\hline MALIGNANT NEOPLASMS & $\begin{array}{l}\text { 140-208, } \\
209.0-209.3 \\
230-234\end{array}$ & 32 & 396 & 0 & 1,394 \\
\hline BENIGN \& UNCERTAIN NEOPLASMS & $\begin{array}{l}209.4-209.6 \\
210-229,235-239\end{array}$ & 0 & 36 & 0 & 116 \\
\hline ENDOCRINE/METABOLIC/IMMUNITY & $240-279$ & 0 & 144 & 10 & 593 \\
\hline BLOOD \& BLOOD-FORMING ORGANS & $280-289$ & 0 & 0 & 0 & 186 \\
\hline MENTAL DISORDERS & 290-319 & 0 & 573 & 123 & 1,628 \\
\hline NERVOUS SYSTEM (NS) \& SENSE ORGANS & 320-389 & 18 & 421 & 313 & 1,709 \\
\hline CIRCULATORY SYSTEM & $390-459$ & 353 & 912 & 264 & 3,650 \\
\hline RESPIRATORY SYSTEM & $460-519$ & 0 & 608 & 217 & 1,334 \\
\hline DIGESTIVE SYSTEM & $520-579$ & 0 & 699 & 268 & 2,149 \\
\hline GENITOURINARY SYSTEM & $580-629$ & 53 & 220 & 167 & 816 \\
\hline SKIN AND SUBCUTANEOUS TISSUE & 680-709 & 0 & 316 & 290 & 930 \\
\hline $\begin{array}{l}\text { MUSCULOSKELETAL \& CONNECTIVE } \\
\text { TISSUE }\end{array}$ & 710-739 & 468 & 3,150 & 1,413 & 9,759 \\
\hline CONGENITAL ANOMALIES & $740-759$ & 0 & 204 & 0 & 307 \\
\hline $\begin{array}{l}\text { SYMPTOMS, SIGNS, \& ILL-DEFINED } \\
\text { CONDITIONS }\end{array}$ & 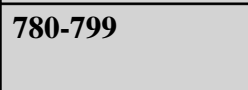 & 73 & 572 & 269 & 1,963 \\
\hline INJURY \& POISONING & $800-999$ & 18 & 1,950 & 1,112 & 5,355 \\
\hline
\end{tabular}

\footnotetext{
*Absences with >1 ICD-9-CM code in the same diagnostic category were counted only once. Only those diagnostic categories and gender/job category combinations with at least one occurrence appear in this table.
} 


\section{Y-12 National Security Complex 2010}

\section{Absence Data}

Appendix K. Age-Adjusted Illness and Injury Rates by Diagnostic Category*

Part 1. Men

\begin{tabular}{|c|c|c|c|c|c|}
\hline & & $\begin{array}{l}\text { Number of } \\
\text { Diagnoses }\end{array}$ & $\begin{array}{c}\text { Age-Adjusted } \\
\text { Rate per } 1,000 * *\end{array}$ & $\begin{array}{c}\text { Lower } 95 \% \\
\text { Confidence } \\
\text { Limit per 1,000 }\end{array}$ & $\begin{array}{c}\text { Upper } 95 \% \\
\text { Confidence } \\
\text { Limit per 1,000 }\end{array}$ \\
\hline Diagnostic Category & ICD-9-CM Code & & & & \\
\hline INFECTIOUS \& PARASITIC DISEASES (DIS) & 001-139 & 12 & 2.5 & 1.1 & 5.4 \\
\hline -Other Bacterial Dis & 030-041 & 5 & 1.6 & 0.5 & 5.0 \\
\hline -Viral Dis with Exanthem & 050-059 & 3 & 0.4 & 0.1 & 1.1 \\
\hline -Other Viral Dis \& Chlamydiae & 070-079 & 3 & 0.4 & 0.1 & 1.1 \\
\hline -Late Effects & 137-139 & 1 & 0.1 & 0.0 & 0.9 \\
\hline MALIGNANT NEOPLASMS & $\begin{array}{l}140-208, \\
209.0-209.3 \\
230-234\end{array}$ & 22 & 3.7 & 2.4 & 5.9 \\
\hline -Digestive \& Peritoneal & $150-159$ & 2 & 0.3 & 0.1 & 1.2 \\
\hline -Respiratory \& Intrathoracic & $160-165$ & 4 & 0.9 & 0.3 & 2.8 \\
\hline -Bone, Connective Tissue, Skin & 170-173, 176 & 2 & 0.5 & 0.1 & 1.9 \\
\hline -Breast & 174-175 & 1 & 0.2 & 0.0 & 1.3 \\
\hline -Genitourinary & $179-189$ & 7 & 1.0 & 0.5 & 2.2 \\
\hline -Nervous System & 190-192 & 1 & 0.2 & 0.0 & 1.3 \\
\hline -Endocrine & $\begin{array}{l}\text { 193-194, } \\
\text { 209.0-209.3 }\end{array}$ & 1 & 0.1 & 0.0 & 0.9 \\
\hline -Lymphatic \& Hematopoietic & $200-208$ & 4 & 0.5 & 0.2 & 1.5 \\
\hline BENIGN \& UNCERTAIN NEOPLASMS & $\begin{array}{l}209.4-209.6 \\
210-229,235-239\end{array}$ & 10 & 1.7 & 0.9 & 3.6 \\
\hline ENDOCRINE/METABOLIC/IMMUNITY & 240-279 & 21 & 4.3 & 2.7 & 6.8 \\
\hline -Thyroid Gland Disorders & $240-246$ & 4 & 1.0 & 0.3 & 2.6 \\
\hline -Other Endocrine Gland Dis & 249-259 & 9 & 1.5 & 0.7 & 3.0 \\
\hline -Other Metabolic \& Immunity Disorders & 270-279 & 8 & 1.9 & 0.9 & 4.0 \\
\hline BLOOD \& BLOOD-FORMING ORGANS & 280-289 & 1 & 0.1 & 0.0 & 0.9 \\
\hline MENTAL DISORDERS & 290-319 & 29 & 12.2 & 8.0 & 18.6 \\
\hline -Psychoses & 290-299 & 14 & 7.2 & 4.0 & 13.1 \\
\hline -Non-Psychotic Disorders & $300-302,306-316$ & 13 & 4.6 & 2.5 & 8.4 \\
\hline -Alcohol Dependence & 303 & 2 & 0.4 & 0.1 & 1.8 \\
\hline NERVOUS SYSTEM (NS) \& SENSE ORGANS & 320-389 & 50 & 12.1 & 8.8 & 16.7 \\
\hline -Organic Sleep Disorders & 327 & 2 & 0.6 & 0.1 & 2.7 \\
\hline -Hereditary/Degenerative Central NS Dis & 330-337 & 3 & 0.4 & 0.1 & 1.3 \\
\hline -Pain & 338 & 2 & 0.6 & 0.1 & 2.7 \\
\hline -Other Disorders of Central NS & 340-349 & 4 & 1.3 & 0.5 & 3.5 \\
\hline -Disorders of Peripheral NS & 350-359 & 12 & 2.0 & 1.1 & 3.7 \\
\hline -Disorders of Eye & 360-379 & 20 & 4.1 & 2.5 & 6.7 \\
\hline -Diseases of Ear \& Mastoid & 380-389 & 7 & 3.2 & 1.5 & 7.0 \\
\hline CIRCULATORY SYSTEM & $390-459$ & 82 & 15.2 & 11.7 & 19.7 \\
\hline
\end{tabular}

(Continued)

* Only those diagnostic categories with at least one occurrence appear in this table.

**Standardized to age distribution of 2000 U.S. population. 


\section{Y-12 National Security Complex 2010}

\section{Absence Data}

Appendix K. Age-Adjusted IIIness and Injury Rates by Diagnostic Category*

Part 1. Men

\begin{tabular}{|c|c|c|c|c|c|}
\hline & & $\begin{array}{l}\text { Number of } \\
\text { Diagnoses }\end{array}$ & $\begin{array}{l}\text { Age-Adjusted } \\
\text { Rate per } 1,000 * *\end{array}$ & $\begin{array}{c}\text { Lower } 95 \% \\
\text { Confidence } \\
\text { Limit per 1,000 }\end{array}$ & $\begin{array}{c}\text { Upper 95\% } \\
\text { Confidence } \\
\text { Limit per 1,000 }\end{array}$ \\
\hline Diagnostic Category & ICD-9-CM Code & & & & \\
\hline -Hypertensive Dis & $401-405$ & 16 & 2.5 & 1.4 & 4.3 \\
\hline -Ischemic Heart Dis & $410-414$ & 24 & 3.5 & 2.3 & 5.3 \\
\hline -Other Heart Dis & $420-429$ & 10 & 2.7 & 1.3 & 5.9 \\
\hline -Cerebrovascular Dis & $430-438$ & 9 & 1.9 & 1.0 & 3.8 \\
\hline -Dis of Arteries \& Capillaries & $440-449$ & 4 & 0.6 & 0.2 & 1.6 \\
\hline -Dis of Veins, Lymphatics, Other & $451-459$ & 19 & 4.0 & 2.3 & 7.0 \\
\hline RESPIRATORY SYSTEM & $460-519$ & 108 & 23.3 & 18.6 & 29.1 \\
\hline -Acute Respiratory Infections & $460-466$ & 32 & 6.4 & 4.3 & 9.3 \\
\hline -Other Dis Upper Respiratory Tract & $470-478$ & 37 & 7.7 & 5.2 & 11.4 \\
\hline -Pneumonia \& Influenza & $480-488$ & 18 & 4.2 & 2.4 & 7.4 \\
\hline -Chronic Obstructive Dis & $490-496$ & 18 & 4.2 & 2.3 & 7.4 \\
\hline -Other Respiratory Dis & $510-519$ & 3 & 0.8 & 0.3 & 2.6 \\
\hline DIGESTIVE SYSTEM & $520-579$ & 77 & 17.9 & 13.7 & 23.3 \\
\hline -Oral Cavity, Saliva Glands, Jaw & $520-529$ & 9 & 2.5 & 1.0 & 6.2 \\
\hline -Esophagus, Stomach, Duodenum & $530-538$ & 7 & 1.1 & 0.5 & 2.5 \\
\hline -Hernias & $550-553$ & 19 & 4.7 & 2.9 & 7.8 \\
\hline -Enteritis, Colitis & $555-558$ & 9 & 1.8 & 0.9 & 3.7 \\
\hline -Other Intestinal Dis & $560-569$ & 16 & 3.4 & 1.9 & 5.9 \\
\hline -Other Digestive Dis & $570-579$ & 17 & 4.3 & 2.5 & 7.3 \\
\hline GENITOURINARY SYSTEM & $580-629$ & 22 & 4.2 & 2.5 & 7.0 \\
\hline -Nephritis, Nephrosis & $580-589$ & 2 & 0.3 & 0.1 & 1.2 \\
\hline -Other Urinary Dis & $590-599$ & 10 & 2.4 & 1.1 & 5.2 \\
\hline -Male Genital Organ Dis & $600-608$ & 10 & 1.5 & 0.8 & 2.9 \\
\hline SKIN AND SUBCUTANEOUS TISSUE & $680-709$ & 24 & 6.0 & 3.7 & 10.0 \\
\hline -Infections & $680-686$ & 15 & 4.4 & 2.4 & 8.3 \\
\hline -Other Inflammatory Conditions & 690-698 & 1 & 0.1 & 0.0 & 0.9 \\
\hline -Other & 700-709 & 8 & 1.5 & 0.7 & 3.3 \\
\hline $\begin{array}{l}\text { MUSCULOSKELETAL \& CONNECTIVE } \\
\text { TISSUE }\end{array}$ & $710-739$ & 248 & 56.8 & 49.0 & 65.9 \\
\hline -Arthropathies & $710-719$ & 80 & 15.6 & 12.2 & 20.0 \\
\hline -Dorsopathies & $720-724$ & 117 & 30.7 & 24.7 & 38.2 \\
\hline -Rheumatism, Excluding Back & $725-729$ & 36 & 7.6 & 5.2 & 11.2 \\
\hline -Other Dis \& Acquired Deformities & $730-739$ & 15 & 2.8 & 1.7 & 4.9 \\
\hline CONGENITAL ANOMALIES & $740-759$ & 5 & 1.1 & 0.5 & 2.8 \\
\hline $\begin{array}{l}\text { SYMPTOMS, SIGNS, \& ILL-DEFINED } \\
\text { CONDITIONS }\end{array}$ & $780-799$ & 70 & 14.9 & 11.3 & 19.6 \\
\hline
\end{tabular}

(Continued)

*Only those diagnostic categories with at least one occurrence appear in this table.

**Standardized to age distribution of 2000 U.S. population. 
Y-12 National Security Complex 2010

Absence Data

Appendix K. Age-Adjusted IIIness and Injury Rates by Diagnostic Category*

Part 1. Men

\begin{tabular}{|c|c|c|c|c|c|}
\hline & & $\begin{array}{l}\text { Number of } \\
\text { Diagnoses }\end{array}$ & $\begin{array}{c}\text { Age-Adjusted } \\
\text { Rate per } 1,000 * *\end{array}$ & $\begin{array}{c}\text { Lower } 95 \% \\
\text { Confidence } \\
\text { Limit per 1,000 }\end{array}$ & $\begin{array}{c}\text { Upper 95\% } \\
\text { Confidence } \\
\text { Limit per 1,000 }\end{array}$ \\
\hline Diagnostic Category & ICD-9-CM Code & \multirow[b]{2}{*}{60} & \multirow[b]{2}{*}{13.0} & \multirow[b]{2}{*}{9.7} & \multirow[b]{2}{*}{17.5} \\
\hline -Symptoms & 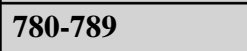 & & & & \\
\hline -Non-Specific Abnormal Findings & \begin{tabular}{|c|}
$790-796$ \\
\end{tabular} & 10 & 1.8 & 0.9 & 3.8 \\
\hline INJURY \& POISONING & $800-999$ & 118 & 33.7 & 26.9 & 42.2 \\
\hline -Fracture - Skull & \begin{tabular}{|l|}
$800-804$ \\
\end{tabular} & 6 & 1.5 & 0.5 & 4.5 \\
\hline -Fracture - Upper Limb & $810-819$ & 10 & 3.9 & 1.9 & 8.0 \\
\hline -Fracture - Lower Limb & $820-829$ & 9 & 1.8 & 0.9 & 4.0 \\
\hline -Dislocation & 830-839 & 19 & 3.6 & 2.2 & 6.0 \\
\hline -Sprains \& Strains - Back & 846-847 & 10 & 1.8 & 0.9 & 3.6 \\
\hline -Sprains \& Strains - Other & $840-845,848$ & 41 & 11.5 & 8.0 & 16.6 \\
\hline -Internal Injury - Thorax, Abdomen, Pelvis & $860-869$ & 1 & 0.8 & 0.1 & 5.5 \\
\hline -Open Wound - Head, Neck, Trunk & $870-879$ & 3 & 1.7 & 0.5 & 6.1 \\
\hline -Superficial Injury & $910-919$ & 1 & 0.8 & 0.1 & 5.5 \\
\hline -Contusion & $920-924$ & 4 & 1.6 & 0.5 & 5.0 \\
\hline -Injury to Nerves \& Spinal Cord & $950-957$ & 1 & 0.3 & 0.0 & 2.0 \\
\hline -Complications \& Unspecified Injuries & 958-959 & 4 & 2.8 & 1.0 & 7.6 \\
\hline -Unspecified Effects - External Causes & $990-995$ & 4 & 0.7 & 0.2 & 2.0 \\
\hline -Complications of Surgical/Medical Care & 996-999 & 5 & 0.8 & 0.3 & 2.1 \\
\hline Total & & 899 & 209.9 & 193.8 & 227.2 \\
\hline
\end{tabular}

* Only those diagnostic categories with at least one occurrence appear in this table.

**Standardized to age distribution of 2000 U.S. population. 


\section{Y-12 National Security Complex 2010}

\section{Absence Data}

Appendix K. Age-Adjusted Illness and Injury Rates by Diagnostic Category*

Part 2. Women

\begin{tabular}{|c|c|c|c|c|c|}
\hline & & $\begin{array}{l}\text { Number of } \\
\text { Diagnoses }\end{array}$ & $\begin{array}{c}\text { Age-Adjusted } \\
\text { Rate per } 1,000 * *\end{array}$ & $\begin{array}{c}\text { Lower } 95 \% \\
\text { Confidence } \\
\text { Limit per 1,000 }\end{array}$ & $\begin{array}{c}\text { Upper } 95 \% \\
\text { Confidence } \\
\text { Limit per 1,000 }\end{array}$ \\
\hline Diagnostic Category & ICD-9-CM Code & & & & \\
\hline INFECTIOUS \& PARASITIC DISEASES (DIS) & 001-139 & 12 & 7.3 & 3.9 & 13.8 \\
\hline -Intestinal Infectious Dis & 001-009 & 4 & 2.1 & 0.7 & 6.1 \\
\hline -Other Bacterial Dis & 030-041 & 3 & 2.5 & 0.7 & 8.8 \\
\hline -Viral Dis with Exanthem & 050-059 & 1 & 0.9 & 0.1 & 6.4 \\
\hline -Other Viral Dis \& Chlamydiae & 070-079 & 2 & 0.9 & 0.2 & 3.7 \\
\hline -Syphilis \& Other Venereal Dis & 090-099 & 1 & 0.3 & 0.0 & 2.2 \\
\hline -Other Infections \& Parasitic Dis & $130-136$ & 1 & 0.6 & 0.1 & 4.0 \\
\hline MALIGNANT NEOPLASMS & $\begin{array}{l}140-208, \\
209.0-209.3 \\
230-234\end{array}$ & 8 & 3.6 & 1.7 & 7.7 \\
\hline -Digestive \& Peritoneal & 150-159 & 2 & 1.2 & 0.3 & 5.6 \\
\hline -Breast & 174-175 & 3 & 1.2 & 0.4 & 3.9 \\
\hline -Genitourinary & 179-189 & 1 & 0.3 & 0.0 & 2.2 \\
\hline -Endocrine & $\begin{array}{l}\text { 193-194, } \\
209.0-209.3\end{array}$ & 1 & 0.3 & 0.0 & 2.2 \\
\hline -Carcinoma in situ & $230-234$ & 1 & 0.6 & 0.1 & 4.0 \\
\hline BENIGN \& UNCERTAIN NEOPLASMS & $\begin{array}{l}209.4-209.6, \\
210-229,235-239\end{array}$ & 7 & 6.9 & 2.8 & 17.1 \\
\hline ENDOCRINE/METABOLIC/IMMUNITY & 240-279 & 10 & 5.7 & 2.7 & 11.8 \\
\hline -Thyroid Gland Disorders & $240-246$ & 2 & 1.2 & 0.3 & 5.6 \\
\hline -Other Endocrine Gland Dis & $249-259$ & 4 & 1.8 & 0.6 & 5.6 \\
\hline -Other Metabolic \& Immunity Disorders & $270-279$ & 4 & 2.6 & 0.8 & 8.5 \\
\hline BLOOD \& BLOOD-FORMING ORGANS & 280-289 & 2 & 1.2 & 0.3 & 5.6 \\
\hline MENTAL DISORDERS & 290-319 & 12 & 12.2 & 5.9 & 25.0 \\
\hline -Psychoses & 290-299 & 4 & 4.6 & 1.4 & 15.1 \\
\hline -Non-Psychotic Disorders & $300-302,306-316$ & 7 & 7.0 & 2.6 & 18.4 \\
\hline -Drug Dependence & $304-305$ & 1 & 0.6 & 0.1 & 4.0 \\
\hline NERVOUS SYSTEM (NS) \& SENSE ORGANS & 320-389 & 37 & 22.8 & 15.9 & 32.8 \\
\hline -Organic Sleep Disorders & 327 & 2 & 1.1 & 0.3 & 4.5 \\
\hline -Hereditary/Degenerative Central NS Dis & 330-337 & 1 & 0.6 & 0.1 & 4.0 \\
\hline -Other Disorders of Central NS & 340-349 & 8 & 7.2 & 3.3 & 15.6 \\
\hline -Disorders of Peripheral NS & $350-359$ & 11 & 5.9 & 3.1 & 11.1 \\
\hline -Disorders of Eye & $360-379$ & 6 & 4.5 & 1.9 & 10.3 \\
\hline -Diseases of Ear \& Mastoid & 380-389 & 9 & 3.6 & 1.8 & 7.1 \\
\hline CIRCULATORY SYSTEM & 390-459 & 23 & 14.3 & 8.7 & 23.6 \\
\hline -Hypertensive Dis & $401-405$ & 4 & 2.7 & 0.9 & 7.6 \\
\hline -Ischemic Heart Dis & $410-414$ & 5 & 3.3 & 1.3 & 8.6 \\
\hline
\end{tabular}

(Continued)

* Only those diagnostic categories with at least one occurrence appear in this table.

**Standardized to age distribution of 2000 U.S. population. 


\section{Y-12 National Security Complex 2010}

\section{Absence Data}

Appendix K. Age-Adjusted Illness and Injury Rates by Diagnostic Category*

Part 2. Women

\begin{tabular}{|c|c|c|c|c|c|}
\hline & & $\begin{array}{l}\text { Number of } \\
\text { Diagnoses }\end{array}$ & $\begin{array}{c}\text { Age-Adjusted } \\
\text { Rate per } 1,000 * *\end{array}$ & $\begin{array}{c}\text { Lower } 95 \% \\
\text { Confidence } \\
\text { Limit per 1,000 }\end{array}$ & $\begin{array}{c}\text { Upper } 95 \% \\
\text { Confidence } \\
\text { Limit per 1,000 }\end{array}$ \\
\hline Diagnostic Category & ICD-9-CM Code & \multirow[b]{2}{*}{1} & \multirow[b]{2}{*}{0.3} & \multirow[b]{2}{*}{0.0} & \multirow[b]{2}{*}{2.2} \\
\hline -Dis of Pulmonary Circulation & $415-417$ & & & & \\
\hline -Other Heart Dis & $420-429$ & 1 & 0.9 & 0.1 & 6.4 \\
\hline -Cerebrovascular Dis & $430-438$ & 3 & 1.5 & 0.4 & 5.5 \\
\hline -Dis of Arteries \& Capillaries & $440-449$ & 2 & 0.6 & 0.2 & 2.5 \\
\hline -Dis of Veins, Lymphatics, Other & 451-459 & 7 & 5.0 & 1.8 & 13.8 \\
\hline RESPIRATORY SYSTEM & $460-519$ & 100 & 70.5 & 54.9 & 90.4 \\
\hline -Acute Respiratory Infections & $460-466$ & 38 & 28.9 & 19.3 & 43.3 \\
\hline -Other Dis Upper Respiratory Tract & $470-478$ & 20 & 15.3 & 8.9 & 26.5 \\
\hline -Pneumonia \& Influenza & $480-488$ & 8 & 6.5 & 2.6 & 16.0 \\
\hline -Chronic Obstructive Dis & $490-496$ & 28 & 16.3 & 10.3 & 25.9 \\
\hline -Other Respiratory Dis & $510-519$ & 6 & 3.5 & 1.3 & 9.1 \\
\hline DIGESTIVE SYSTEM & $520-579$ & 50 & 30.3 & 21.5 & 42.6 \\
\hline -Oral Cavity, Saliva Glands, Jaw & $520-529$ & 9 & 6.1 & 2.8 & 13.2 \\
\hline -Esophagus, Stomach, Duodenum & $530-538$ & 6 & 2.7 & 1.1 & 6.6 \\
\hline -Appendicitis & $540-543$ & 2 & 2.0 & 0.4 & 8.9 \\
\hline -Hernias & $550-553$ & 2 & 1.5 & 0.4 & 6.1 \\
\hline -Enteritis, Colitis & $555-558$ & 6 & 4.3 & 1.6 & 11.4 \\
\hline -Other Intestinal Dis & $560-569$ & 9 & 4.5 & 2.2 & 9.3 \\
\hline -Other Digestive Dis & $570-579$ & 16 & 9.2 & 4.7 & 18.0 \\
\hline GENITOURINARY SYSTEM & $580-629$ & 47 & 35.3 & 24.9 & 50.0 \\
\hline -Nephritis, Nephrosis & $580-589$ & 3 & 2.1 & 0.6 & 7.1 \\
\hline -Other Urinary Dis & $590-599$ & 6 & 3.2 & 1.2 & 8.7 \\
\hline -Breast Disorders & $610-612$ & 4 & 2.0 & 0.7 & 5.5 \\
\hline -Other Female Disorders & $617-629$ & 34 & 27.9 & 18.5 & 42.1 \\
\hline SKIN AND SUBCUTANEOUS TISSUE & 680-709 & 4 & 1.8 & 0.6 & 4.9 \\
\hline -Infections & $680-686$ & 1 & 0.6 & 0.1 & 4.0 \\
\hline -Other Inflammatory Conditions & $690-698$ & 1 & 0.3 & 0.0 & 2.2 \\
\hline -Other & $700-709$ & 2 & 0.9 & 0.2 & 3.7 \\
\hline $\begin{array}{l}\text { MUSCULOSKELETAL \& CONNECTIVE } \\
\text { TISSUE }\end{array}$ & $710-739$ & 121 & 77.1 & 62.6 & 95.0 \\
\hline -Arthropathies & 710-719 & 36 & 19.2 & 13.4 & 27.5 \\
\hline -Dorsopathies & $720-724$ & 47 & 35.7 & 25.5 & 50.0 \\
\hline -Rheumatism, Excluding Back & 725-729 & 24 & 14.8 & 9.4 & 23.4 \\
\hline -Other Dis \& Acquired Deformities & 730-739 & 14 & 7.4 & 4.0 & 13.6 \\
\hline CONGENITAL ANOMALIES & $740-759$ & 3 & 1.2 & 0.4 & 3.9 \\
\hline
\end{tabular}

(Continued)

*Only those diagnostic categories with at least one occurrence appear in this table.

**Standardized to age distribution of 2000 U.S. population. 
Y-12 National Security Complex 2010

Absence Data

Appendix K. Age-Adjusted IIlness and Injury Rates by Diagnostic Category*

Part 2. Women

\begin{tabular}{|c|c|c|c|c|c|}
\hline & & $\begin{array}{l}\text { Number of } \\
\text { Diagnoses }\end{array}$ & $\begin{array}{c}\text { Age-Adjusted } \\
\text { Rate per } 1,000 * *\end{array}$ & $\begin{array}{c}\text { Lower } 95 \% \\
\text { Confidence } \\
\text { Limit per 1,000 }\end{array}$ & $\begin{array}{c}\text { Upper } 95 \% \\
\text { Confidence } \\
\text { Limit per 1,000 }\end{array}$ \\
\hline Diagnostic Category & ICD-9-CM Code & & & & \\
\hline $\begin{array}{l}\text { SYMPTOMS, SIGNS, \& ILL-DEFINED } \\
\text { CONDITIONS }\end{array}$ & \begin{tabular}{|l|}
$780-799$ \\
\end{tabular} & 53 & 35.3 & 25.5 & 48.8 \\
\hline -Symptoms & $780-789$ & 51 & 34.1 & 24.5 & 47.5 \\
\hline -Non-Specific Abnormal Findings & $790-796$ & 2 & 1.2 & 0.3 & $\overline{5.6}$ \\
\hline INJURY \& POISONING & \begin{tabular}{|l|}
$800-999$ \\
\end{tabular} & 38 & 24.8 & 16.9 & 36.4 \\
\hline -Fracture - Skull & \begin{tabular}{|l|}
$800-804$ \\
\end{tabular} & 2 & 1.5 & 0.4 & 6.1 \\
\hline -Fracture - Upper Limb & \begin{tabular}{|l|}
$810-819$ \\
\end{tabular} & 5 & 1.6 & 0.7 & 3.8 \\
\hline -Fracture - Lower Limb & $820-829$ & 3 & 4.2 & 1.4 & 13.0 \\
\hline -Dislocation & $830-839$ & 6 & 2.4 & 1.0 & 5.5 \\
\hline -Sprains \& Strains - Back & 846-847 & 3 & 1.8 & 0.5 & 6.0 \\
\hline -Sprains \& Strains - Other & $840-845,848$ & 7 & 4.7 & 2.2 & 10.2 \\
\hline -Intracranial Injury & $850-854$ & 1 & 0.6 & 0.1 & 4.0 \\
\hline -Open Wound - Head, Neck, Trunk & \begin{tabular}{|l|}
$870-879$ \\
\end{tabular} & 3 & 2.6 & 0.7 & 9.3 \\
\hline -Contusion & $920-924$ & 2 & 2.9 & 0.6 & 14.6 \\
\hline -Burns & $940-949$ & 1 & 0.3 & 0.0 & 2.2 \\
\hline -Complications \& Unspecified Injuries & 958-959 & 1 & 0.6 & 0.1 & 4.0 \\
\hline -Complications of Surgical/Medical Care & 996-999 & 4 & 1.8 & 0.6 & 4.9 \\
\hline Total & & 527 & 350.2 & 315.5 & 388.8 \\
\hline
\end{tabular}

* Only those diagnostic categories with at least one occurrence appear in this table.

**Standardized to age distribution of 2000 U.S. population. 


\section{Y-12 National Security Complex 2010}

\section{Absence Data}

Appendix K. Age-Adjusted Illness and Injury Rates by Diagnostic Category*

Part 3. Men and Women

\begin{tabular}{|c|c|c|c|c|c|}
\hline & & $\begin{array}{l}\text { Number of } \\
\text { Diagnoses }\end{array}$ & $\begin{array}{c}\text { Age-Adjusted } \\
\text { Rate per } 1,000 * *\end{array}$ & $\begin{array}{c}\text { Lower } 95 \% \\
\text { Confidence } \\
\text { Limit per 1,000 }\end{array}$ & $\begin{array}{c}\text { Upper } 95 \% \\
\text { Confidence } \\
\text { Limit per 1,000 }\end{array}$ \\
\hline Diagnostic Category & ICD-9-CM Code & & & & \\
\hline INFECTIOUS \& PARASITIC DISEASES (DIS) & 001-139 & 24 & 3.8 & 2.3 & 6.2 \\
\hline -Intestinal Infectious Dis & 001-009 & 4 & 0.5 & 0.2 & 1.5 \\
\hline -Other Bacterial Dis & 030-041 & 8 & 1.9 & 0.9 & 4.4 \\
\hline -Viral Dis with Exanthem & 050-059 & 4 & 0.4 & 0.2 & 1.2 \\
\hline -Other Viral Dis \& Chlamydiae & 070-079 & 5 & 0.5 & 0.2 & 1.4 \\
\hline -Syphilis \& Other Venereal Dis & 090-099 & 1 & 0.1 & 0.0 & 0.6 \\
\hline -Other Infections \& Parasitic Dis & $130-136$ & 1 & 0.2 & 0.0 & 1.3 \\
\hline -Late Effects & 137-139 & 1 & 0.1 & 0.0 & 0.6 \\
\hline MALIGNANT NEOPLASMS & $\begin{array}{l}140-208, \\
209.0-209.3 \\
230-234\end{array}$ & 30 & 3.9 & 2.7 & 5.8 \\
\hline -Digestive \& Peritoneal & $150-159$ & 4 & 0.5 & 0.2 & 1.4 \\
\hline -Respiratory \& Intrathoracic & $160-165$ & 4 & 0.7 & 0.2 & 2.2 \\
\hline -Bone, Connective Tissue, Skin & $170-173,176$ & 2 & 0.3 & 0.1 & 1.4 \\
\hline -Breast & 174-175 & 4 & 0.5 & 0.2 & 1.5 \\
\hline -Genitourinary & 179-189 & 8 & 0.9 & 0.4 & 1.9 \\
\hline -Nervous System & 190-192 & 1 & 0.2 & 0.0 & 1.1 \\
\hline -Endocrine & $\begin{array}{l}\text { 193-194, } \\
\text { 209.0-209.3 }\end{array}$ & 2 & 0.2 & 0.0 & 0.7 \\
\hline -Lymphatic \& Hematopoietic & $200-208$ & 4 & 0.4 & 0.2 & 1.2 \\
\hline -Carcinoma in situ & $230-234$ & 1 & 0.2 & 0.0 & 1.3 \\
\hline BENIGN \& UNCERTAIN NEOPLASMS & $\begin{array}{l}\text { 209.4-209.6, } \\
210-229,235-239\end{array}$ & 17 & 3.1 & 1.7 & 5.6 \\
\hline ENDOCRINE/METABOLIC/IMMUNITY & $240-279$ & 31 & 4.4 & 3.0 & 6.5 \\
\hline -Thyroid Gland Disorders & $240-246$ & 6 & 0.9 & 0.4 & 2.1 \\
\hline -Other Endocrine Gland Dis & $249-259$ & 13 & 1.5 & 0.8 & 2.6 \\
\hline -Other Metabolic \& Immunity Disorders & 270-279 & 12 & 2.1 & 1.1 & 3.9 \\
\hline BLOOD \& BLOOD-FORMING ORGANS & $280-289$ & 3 & 0.3 & 0.1 & 1.1 \\
\hline MENTAL DISORDERS & 290-319 & 41 & 12.3 & 8.6 & 17.6 \\
\hline -Psychoses & 290-299 & 18 & 6.5 & 3.8 & 11.1 \\
\hline -Non-Psychotic Disorders & $300-302,306-316$ & 20 & 5.3 & 3.2 & 8.8 \\
\hline -Alcohol Dependence & 303 & 2 & 0.3 & 0.1 & 1.2 \\
\hline -Drug Dependence & 304-305 & 1 & 0.2 & 0.0 & 1.3 \\
\hline NERVOUS SYSTEM (NS) \& SENSE ORGANS & 320-389 & 87 & 15.0 & 11.8 & 19.1 \\
\hline -Organic Sleep Disorders & 327 & 4 & 0.8 & 0.3 & 2.3 \\
\hline -Hereditary/Degenerative Central NS Dis & 330-337 & 4 & 0.5 & 0.2 & 1.5 \\
\hline -Pain & 338 & 2 & 0.4 & 0.1 & 2.1 \\
\hline
\end{tabular}

(Continued)

* Only those diagnostic categories with at least one occurrence appear in this table.

**Standardized to age distribution of 2000 U.S. population. 


\section{Y-12 National Security Complex 2010}

\section{Absence Data}

Appendix K. Age-Adjusted Illness and Injury Rates by Diagnostic Category*

Part 3. Men and Women

\begin{tabular}{|c|c|c|c|c|c|}
\hline & & $\begin{array}{l}\text { Number of } \\
\text { Diagnoses }\end{array}$ & $\begin{array}{c}\text { Age-Adjusted } \\
\text { Rate per } 1,000 * *\end{array}$ & $\begin{array}{c}\text { Lower } 95 \% \\
\text { Confidence } \\
\text { Limit per 1,000 }\end{array}$ & $\begin{array}{c}\text { Upper } 95 \% \\
\text { Confidence } \\
\text { Limit per 1,000 }\end{array}$ \\
\hline Diagnostic Category & ICD-9-CM Code & \multirow[b]{2}{*}{12} & \multirow[b]{2}{*}{2.6} & \multirow[b]{2}{*}{1.4} & \multirow[b]{2}{*}{4.7} \\
\hline -Other Disorders of Central NS & $340-349$ & & & & \\
\hline -Disorders of Peripheral NS & $350-359$ & 23 & 3.1 & 2.0 & 4.8 \\
\hline -Disorders of Eye & $360-379$ & 26 & 4.1 & 2.7 & 6.3 \\
\hline -Diseases of Ear \& Mastoid & $380-389$ & 16 & 3.5 & 2.0 & 6.2 \\
\hline CIRCULATORY SYSTEM & $390-459$ & 105 & 15.0 & 11.9 & 18.8 \\
\hline -Hypertensive Dis & $401-405$ & 20 & 2.5 & 1.6 & 4.1 \\
\hline -Ischemic Heart Dis & $410-414$ & 29 & 3.3 & 2.3 & 4.9 \\
\hline -Dis of Pulmonary Circulation & $415-417$ & 1 & 0.1 & 0.0 & $\overline{0.6}$ \\
\hline -Other Heart Dis & $420-429$ & 11 & 2.3 & 1.1 & 4.7 \\
\hline -Cerebrovascular Dis & $430-438$ & 12 & 1.7 & 1.0 & 3.1 \\
\hline -Dis of Arteries \& Capillaries & $440-449$ & 6 & 0.7 & 0.3 & 1.5 \\
\hline -Dis of Veins, Lymphatics, Other & 451-459 & 26 & 4.4 & 2.7 & 7.1 \\
\hline RESPIRATORY SYSTEM & $460-519$ & 208 & 35.2 & 29.8 & 41.5 \\
\hline -Acute Respiratory Infections & $460-466$ & 70 & 12.2 & 9.2 & 16.1 \\
\hline -Other Dis Upper Respiratory Tract & $470-478$ & 57 & 9.6 & 7.0 & 13.2 \\
\hline -Pneumonia \& Influenza & $480-488$ & 26 & 4.7 & 2.9 & 7.7 \\
\hline -Chronic Obstructive Dis & $490-496$ & 46 & 7.2 & 5.0 & 10.2 \\
\hline -Other Respiratory Dis & $510-519$ & 9 & 1.5 & 0.8 & 3.1 \\
\hline DIGESTIVE SYSTEM & $520-579$ & 127 & 21.2 & 17.3 & 26.0 \\
\hline -Oral Cavity, Saliva Glands, Jaw & $520-529$ & 18 & 3.5 & 1.9 & 6.3 \\
\hline -Esophagus, Stomach, Duodenum & $530-538$ & 13 & 1.5 & 0.9 & 2.7 \\
\hline -Appendicitis & $540-543$ & 2 & 0.5 & 0.1 & 2.2 \\
\hline -Hernias & $550-553$ & 21 & 3.9 & 2.5 & 6.3 \\
\hline -Enteritis, Colitis & $555-558$ & 15 & 2.4 & 1.4 & 4.3 \\
\hline -Other Intestinal Dis & $560-569$ & 25 & 3.7 & 2.4 & 5.7 \\
\hline -Other Digestive Dis & $570-579$ & 33 & 5.6 & 3.7 & 8.5 \\
\hline GENITOURINARY SYSTEM & $580-629$ & 69 & 12.2 & 9.2 & 16.2 \\
\hline -Nephritis, Nephrosis & $580-589$ & 5 & 0.6 & 0.3 & 1.6 \\
\hline -Other Urinary Dis & $590-599$ & 16 & 2.6 & 1.4 & $\overline{4.9}$ \\
\hline -Male Genital Organ Dis & $600-608$ & 10 & 1.2 & 0.6 & 2.3 \\
\hline -Breast Disorders & $610-612$ & 4 & 0.6 & 0.2 & 1.8 \\
\hline -Other Female Disorders & $617-629$ & 34 & 7.0 & 4.7 & 10.5 \\
\hline SKIN AND SUBCUTANEOUS TISSUE & $680-709$ & 28 & 5.0 & 3.2 & 8.0 \\
\hline -Infections & $680-686$ & 16 & 3.5 & 1.9 & 6.3 \\
\hline -Other Inflammatory Conditions & 690-698 & 2 & 0.2 & 0.0 & 0.7 \\
\hline
\end{tabular}

(Continued)

*Only those diagnostic categories with at least one occurrence appear in this table.

**Standardized to age distribution of 2000 U.S. population. 


\section{Y-12 National Security Complex 2010}

\section{Absence Data}

Appendix K. Age-Adjusted Illness and Injury Rates by Diagnostic Category*

Part 3. Men and Women

\begin{tabular}{|c|c|c|c|c|c|}
\hline & & $\begin{array}{c}\text { Number of } \\
\text { Diagnoses }\end{array}$ & $\begin{array}{c}\text { Age-Adjusted } \\
\text { Rate per } 1,000 * *\end{array}$ & $\begin{array}{c}\text { Lower } 95 \% \\
\text { Confidence } \\
\text { Limit per 1,000 }\end{array}$ & $\begin{array}{c}\text { Upper } 95 \% \\
\text { Confidence } \\
\text { Limit per 1,000 }\end{array}$ \\
\hline Diagnostic Category & ICD-9-CM Code & \multirow[b]{2}{*}{10} & \multirow[b]{2}{*}{1.4} & \multirow[b]{2}{*}{0.7} & \multirow[b]{2}{*}{2.8} \\
\hline -Other & 700-709 & & & & \\
\hline $\begin{array}{l}\text { MUSCULOSKELETAL \& CONNECTIVE } \\
\text { TISSUE }\end{array}$ & 710-739 & 369 & 61.8 & 54.9 & 69.7 \\
\hline -Arthropathies & 710-719 & 116 & 16.5 & 13.5 & 20.2 \\
\hline -Dorsopathies & $720-724$ & 164 & 32.1 & 26.8 & 38.5 \\
\hline -Rheumatism, Excluding Back & 725-729 & 60 & 9.2 & 6.8 & 12.3 \\
\hline -Other Dis \& Acquired Deformities & 730-739 & 29 & 4.0 & 2.7 & 5.9 \\
\hline CONGENITAL ANOMALIES & $740-759$ & 8 & 1.2 & 0.6 & 2.4 \\
\hline $\begin{array}{l}\text { SYMPTOMS, SIGNS, \& ILL-DEFINED } \\
\text { CONDITIONS }\end{array}$ & 780-799 & 123 & 20.0 & 16.3 & 24.6 \\
\hline -Symptoms & 780-789 & 111 & 18.4 & 14.8 & 22.9 \\
\hline -Non-Specific Abnormal Findings & $790-796$ & 12 & 1.6 & 0.8 & 3.0 \\
\hline INJURY \& POISONING & $800-999$ & 156 & 31.4 & 25.8 & 38.1 \\
\hline -Fracture - Skull & $800-804$ & 8 & 1.5 & 0.6 & 3.7 \\
\hline -Fracture - Upper Limb & 810-819 & 15 & 3.3 & 1.8 & 6.3 \\
\hline -Fracture - Lower Limb & $820-829$ & 12 & 2.4 & 1.3 & 4.6 \\
\hline -Dislocation & 830-839 & 25 & 3.3 & 2.1 & 5.2 \\
\hline -Sprains \& Strains - Back & 846-847 & 13 & 1.7 & 1.0 & 3.0 \\
\hline -Sprains \& Strains - Other & $840-845,848$ & 48 & 9.7 & 6.9 & 13.5 \\
\hline -Intracranial Injury & $850-854$ & 1 & 0.2 & 0.0 & 1.3 \\
\hline -Internal Injury - Thorax, Abdomen, Pelvis & $860-869$ & 1 & 0.6 & 0.1 & 4.1 \\
\hline -Open Wound - Head, Neck, Trunk & 870-879 & 6 & 1.9 & 0.7 & 4.9 \\
\hline -Superficial Injury & 910-919 & 1 & 0.6 & 0.1 & 4.1 \\
\hline -Contusion & $920-924$ & 6 & 2.0 & 0.8 & 5.0 \\
\hline -Burns & $940-949$ & 1 & 0.1 & 0.0 & 0.6 \\
\hline -Injury to Nerves \& Spinal Cord & 950-957 & 1 & 0.2 & 0.0 & 1.3 \\
\hline -Complications \& Unspecified Injuries & 958-959 & 5 & 2.2 & 0.9 & 5.7 \\
\hline -Unspecified Effects - External Causes & $990-995$ & 4 & 0.5 & 0.2 & 1.5 \\
\hline -Complications of Surgical/Medical Care & 996-999 & 9 & 1.2 & 0.6 & 2.3 \\
\hline Total & & 1426 & 245.8 & 230.9 & 261.7 \\
\hline
\end{tabular}

* Only those diagnostic categories with at least one occurrence appear in this table.

**Standardized to age distribution of 2000 U.S. population. 


\section{Y-12 National Security Complex 2010}

OSHA Data

Appendix L. Number of Workers with at Least One OSHA Event by Gender, Age, and Job Category*

\begin{tabular}{|c|c|c|c|c|c|c|c|c|c|c|}
\hline \multirow{3}{*}{$\begin{array}{c}\text { Job } \\
\text { Category }\end{array}$} & \multicolumn{4}{|c|}{ Women } & \multicolumn{5}{|c|}{ Men } & \multirow[b]{3}{*}{ TOTAL } \\
\hline & \multicolumn{3}{|c|}{ Age Group } & \multirow[b]{2}{*}{ TOTAL } & \multicolumn{4}{|c|}{ Age Group } & \multirow[b]{2}{*}{ TOTAL } & \\
\hline & $30-39$ & $40-49$ & $50+$ & & $16-29$ & $30-39$ & $40-49$ & $50+$ & & \\
\hline Professional & 0 & 1 & 0 & 1 & 0 & 0 & 0 & 1 & 1 & 2 \\
\hline Service & 1 & 3 & 2 & 6 & 2 & 3 & 2 & 6 & 13 & 19 \\
\hline Crafts & 2 & 1 & 6 & 9 & 4 & 3 & 9 & 18 & 34 & 43 \\
\hline TOTAL & 3 & 5 & 8 & 16 & 6 & 6 & 11 & 25 & 48 & 64 \\
\hline
\end{tabular}

*Only those job categories and gender/age combinations with at least one OSHA event appear in this table.

Appendix M. Total Number of Workdays Lost or with Restricted Activity from OSHA Events by Gender and Age

\begin{tabular}{|c|c|c|c|c|c|c|c|c|c|}
\hline \multirow[b]{2}{*}{ Age Group } & \multicolumn{3}{|c|}{ Women } & \multicolumn{3}{|c|}{ Men } & \multicolumn{3}{|c|}{ TOTAL } \\
\hline & $\begin{array}{c}\text { Number of } \\
\text { Events }\end{array}$ & $\begin{array}{c}\text { Days } \\
\text { Restricted }\end{array}$ & Days Lost & $\begin{array}{c}\text { Number of } \\
\text { Events }\end{array}$ & \begin{tabular}{|c|} 
Days \\
Restricted
\end{tabular} & Days Lost & $\begin{array}{c}\text { Number of } \\
\text { Events }\end{array}$ & $\begin{array}{c}\text { Days } \\
\text { Restricted }\end{array}$ & Days Lost \\
\hline $16-29$ & 0 & 0 & 0 & 6 & 20 & 0 & 6 & 20 & 0 \\
\hline $30-39$ & 3 & 0 & 0 & 6 & 136 & 84 & 9 & 136 & 84 \\
\hline $40-49$ & 5 & 16 & 0 & 11 & 320 & 157 & 16 & 336 & 157 \\
\hline $50+$ & 8 & 79 & 170 & 25 & 328 & 75 & 33 & 407 & 245 \\
\hline TOTAL & 16 & 95 & 170 & 48 & 804 & 316 & 64 & 899 & 486 \\
\hline
\end{tabular}

Appendix N. Total Number of Workdays Lost or with Restricted Activity from OSHA Events by Gender and Job Category*

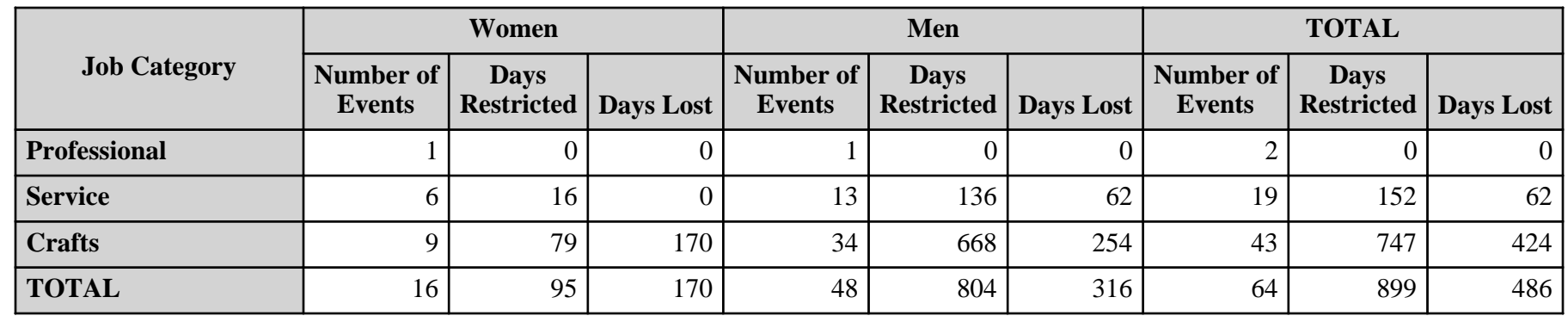

*Only those job categories with at least one OSHA event appear in this table. 
Y-12 National Security Complex 2010

OSHA Data

Appendix O. Number of Diagnoses in Each Diagnostic Category by Gender and Age*

\begin{tabular}{|c|c|c|c|c|c|}
\hline & & \multicolumn{4}{|c|}{ Women } \\
\hline & & \multicolumn{3}{|c|}{ Age Group } & \multirow[b]{2}{*}{ TOTAL } \\
\hline & & $30-39$ & $40-49$ & $50+$ & \\
\hline Diagnostic Category & ICD-9-CM code & \multirow[b]{2}{*}{0} & \multirow[b]{2}{*}{0} & \multirow[b]{2}{*}{0} & \multirow[b]{2}{*}{0} \\
\hline NERVOUS SYSTEM (NS) \& SENSE ORGANS & $320-389$ & & & & \\
\hline -Disorders of Eye & $360-379$ & 0 & 0 & 0 & 0 \\
\hline RESPIRATORY SYSTEM & $460-519$ & 0 & 0 & 0 & 0 \\
\hline -Other Dis Upper Respiratory Tract & $470-478$ & 0 & 0 & 0 & 0 \\
\hline -Lung Dis from External Agents & $500-508$ & 0 & 0 & 0 & 0 \\
\hline $\begin{array}{l}\text { MUSCULOSKELETAL \& CONNECTIVE } \\
\text { TISSUE }\end{array}$ & 710-739 & 0 & 0 & 1 & 1 \\
\hline -Arthropathies & $710-719$ & 0 & 0 & 1 & 1 \\
\hline -Dorsopathies & $720-724$ & 0 & 0 & 0 & 0 \\
\hline -Rheumatism, Excluding Back & $725-729$ & 0 & 0 & 0 & 0 \\
\hline $\begin{array}{l}\text { SYMPTOMS, SIGNS, \& ILL-DEFINED } \\
\text { CONDITIONS }\end{array}$ & 780-799 & 0 & 0 & 1 & 1 \\
\hline -Symptoms & 780-789 & 0 & 0 & 1 & 1 \\
\hline INJURY \& POISONING & $800-999$ & 4 & 7 & 19 & 30 \\
\hline -Fracture - Neck, Trunk & 805-809 & 0 & 0 & 0 & 0 \\
\hline -Fracture - Upper Limb & 810-819 & 0 & 0 & 1 & 1 \\
\hline -Fracture - Lower Limb & 820-829 & 0 & 0 & 0 & 0 \\
\hline -Dislocation & 830-839 & 0 & 0 & 1 & 1 \\
\hline -Sprains \& Strains - Back & 846-847 & 0 & 0 & 1 & 1 \\
\hline -Sprains \& Strains - Other & $840-845,848$ & 1 & 2 & 2 & 5 \\
\hline -Intracranial Injury & $850-854$ & 0 & 1 & 0 & 1 \\
\hline -Open Wound - Head, Neck, Trunk & $870-879$ & 0 & 0 & 2 & 2 \\
\hline -Open Wound - Upper Limb & 880-887 & 1 & 0 & 1 & 2 \\
\hline -Open Wound - Lower Limb & $890-897$ & 0 & 0 & 0 & 0 \\
\hline -Superficial Injury & 910-919 & 1 & 0 & 1 & 2 \\
\hline -Contusion & $920-924$ & 1 & 3 & 9 & 13 \\
\hline -Crushing Injury & 925-929 & 0 & 0 & 0 & 0 \\
\hline -Foreign Body Entering Orifice & 930-939 & 0 & 1 & 0 & 1 \\
\hline -Burns & $940-949$ & 0 & 0 & 0 & 0 \\
\hline -Complications \& Unspecified Injuries & 958-959 & 0 & 0 & 1 & 1 \\
\hline -Toxic Effects - Non-medicinal & 980-989 & 0 & 0 & 0 & 0 \\
\hline -Unspecified Effects - External Causes & $990-995$ & 0 & 0 & 0 & 0 \\
\hline
\end{tabular}

*Only those diagnostic categories and gender/age combinations with at least one OSHA event appear in this table. 
Y-12 National Security Complex 2010

OSHA Data

Appendix O. Number of Diagnoses in Each Diagnostic Category by Gender and Age*

\begin{tabular}{|c|c|c|c|c|c|c|c|}
\hline & & \multicolumn{5}{|c|}{ Men } & \multirow[b]{3}{*}{ TOTAL } \\
\hline & & \multicolumn{4}{|c|}{ Age Group } & \multirow[b]{2}{*}{ TOTAL } & \\
\hline & & $16-29$ & 30 - 39 & $40-49$ & $50+$ & & \\
\hline Diagnostic Category & ICD-9-CM code & & & & & & \\
\hline NERVOUS SYSTEM (NS) \& SENSE ORGANS & $320-389$ & 0 & 0 & 0 & 2 & 2 & 2 \\
\hline -Disorders of Eye & $360-379$ & 0 & 0 & 0 & 2 & 2 & 2 \\
\hline RESPIRATORY SYSTEM & $460-519$ & 0 & 0 & 1 & 3 & 4 & 4 \\
\hline -Other Dis Upper Respiratory Tract & $470-478$ & 0 & 0 & 0 & 1 & 1 & 1 \\
\hline -Lung Dis from External Agents & $500-508$ & 0 & 0 & 1 & 2 & 3 & 3 \\
\hline $\begin{array}{l}\text { MUSCULOSKELETAL \& CONNECTIVE } \\
\text { TISSUE }\end{array}$ & 710-739 & 3 & 1 & 5 & 7 & 16 & 17 \\
\hline -Arthropathies & $710-719$ & 0 & 0 & 1 & 4 & 5 & 6 \\
\hline -Dorsopathies & $720-724$ & 3 & 1 & 4 & 1 & 9 & 9 \\
\hline -Rheumatism, Excluding Back & $725-729$ & 0 & 0 & 0 & 2 & 2 & 2 \\
\hline $\begin{array}{l}\text { SYMPTOMS, SIGNS, \& ILL-DEFINED } \\
\text { CONDITIONS }\end{array}$ & 780-799 & 0 & 0 & 0 & 4 & 4 & 5 \\
\hline -Symptoms & 780-789 & 0 & 0 & 0 & 4 & 4 & 5 \\
\hline INJURY \& POISONING & $800-999$ & 8 & 6 & 12 & 32 & 58 & 88 \\
\hline -Fracture - Neck, Trunk & 805-809 & 0 & 0 & 0 & 1 & 1 & 1 \\
\hline -Fracture - Upper Limb & 810-819 & 0 & 0 & 0 & 3 & 3 & 4 \\
\hline -Fracture - Lower Limb & 820-829 & 0 & 0 & 0 & 1 & 1 & 1 \\
\hline -Dislocation & 830-839 & 0 & 0 & 0 & 2 & 2 & 3 \\
\hline -Sprains \& Strains - Back & 846-847 & 1 & 1 & 3 & 1 & 6 & 7 \\
\hline -Sprains \& Strains - Other & $840-845,848$ & 1 & 2 & 2 & 5 & 10 & 15 \\
\hline -Intracranial Injury & $850-854$ & 0 & 2 & 0 & 0 & 2 & 3 \\
\hline -Open Wound - Head, Neck, Trunk & $870-879$ & 0 & 0 & 0 & 1 & 1 & 3 \\
\hline -Open Wound - Upper Limb & 880-887 & 1 & 0 & 1 & 3 & 5 & 7 \\
\hline -Open Wound - Lower Limb & $890-897$ & 1 & 0 & 0 & 1 & 2 & 2 \\
\hline -Superficial Injury & 910-919 & 0 & 0 & 0 & 3 & 3 & 5 \\
\hline -Contusion & $920-924$ & 2 & 0 & 1 & 6 & 9 & 22 \\
\hline -Crushing Injury & 925-929 & 0 & 0 & 1 & 0 & 1 & 1 \\
\hline -Foreign Body Entering Orifice & 930-939 & 1 & 1 & 1 & 2 & 5 & 6 \\
\hline -Burns & 940-949 & 0 & 0 & 2 & 0 & 2 & 2 \\
\hline -Complications \& Unspecified Injuries & 958-959 & 0 & 0 & 0 & 0 & 0 & 1 \\
\hline -Toxic Effects - Non-medicinal & 980-989 & 1 & 0 & 1 & 2 & 4 & 4 \\
\hline -Unspecified Effects - External Causes & $990-995$ & 0 & 0 & 0 & 1 & 1 & 1 \\
\hline
\end{tabular}

*Only those diagnostic categories and gender/age combinations with at least one OSHA event appear in this table. 
Y-12 National Security Complex 2010

OSHA Data

Appendix O. Number of Diagnoses in Each Diagnostic Category by Gender and Age*

\begin{tabular}{|c|c|c|c|c|c|c|c|c|c|c|}
\hline & \multicolumn{4}{|c|}{ Women } & \multicolumn{5}{|c|}{ Men } & \multirow[b]{3}{*}{ TOTAL } \\
\hline & \multicolumn{3}{|c|}{ Age Group } & \multirow[b]{2}{*}{ TOTAL } & \multicolumn{4}{|c|}{ Age Group } & \multirow[b]{2}{*}{ TOTAL } & \\
\hline & $30-39$ & $40-49$ & $50+$ & & $16-29$ & 30 - 39 & $40-49$ & $50+$ & & \\
\hline Diagnostic Category & \multirow[b]{2}{*}{4} & \multirow[b]{2}{*}{7} & \multirow[b]{2}{*}{21} & \multirow[b]{2}{*}{32} & \multirow[b]{2}{*}{11} & \multirow[b]{2}{*}{7} & \multirow[b]{2}{*}{18} & \multirow[b]{2}{*}{48} & \multirow[b]{2}{*}{84} & \multirow[b]{2}{*}{116} \\
\hline Total & & & & & & & & & & \\
\hline
\end{tabular}

*Only those diagnostic categories and gender/age combinations with at least one OSHA event appear in this table. 


\section{Y-12 National Security Complex 2010}

OSHA Data

Appendix P. Number of Workdays Lost or with Restricted Activity in Each Diagnostic Category by Gender and Age*

\begin{tabular}{|c|c|c|c|c|c|c|c|}
\hline & & \multicolumn{6}{|c|}{ Women } \\
\hline & & \multicolumn{6}{|c|}{ Age Group } \\
\hline & & \multicolumn{2}{|c|}{$30-39$} & \multicolumn{2}{|c|}{$40-49$} & \multicolumn{2}{|c|}{$50+$} \\
\hline & & $\begin{array}{c}\text { Days } \\
\text { Restricted }\end{array}$ & Days Lost & $\begin{array}{c}\text { Days } \\
\text { Restricted }\end{array}$ & Days Lost & $\begin{array}{c}\text { Days } \\
\text { Restricted }\end{array}$ & Days Lost \\
\hline Diagnostic Category & ICD-9-CM Codes & \multirow[b]{2}{*}{0} & \multirow[b]{2}{*}{0} & \multirow[b]{2}{*}{0} & \multirow[b]{2}{*}{0} & \multirow[b]{2}{*}{0} & \multirow[b]{2}{*}{0} \\
\hline -Arthropathies & $710-719$ & & & & & & \\
\hline -Symptoms & $780-789$ & 0 & 0 & 0 & 0 & 75 & 11 \\
\hline -Fracture - Upper Limb & \begin{tabular}{|l|}
$810-819$ \\
\end{tabular} & 0 & 0 & 0 & 0 & 0 & 157 \\
\hline -Dislocation & \begin{tabular}{|l|}
$830-839$ \\
\end{tabular} & 0 & 0 & 0 & 0 & 0 & 157 \\
\hline -Sprains \& Strains - Back & $846-847$ & 0 & 0 & 0 & 0 & 75 & 11 \\
\hline -Sprains \& Strains - Other & $840-845,848$ & 0 & 0 & 0 & 0 & 0 & 0 \\
\hline -Intracranial Injury & $850-854$ & 0 & 0 & 0 & 0 & 0 & 0 \\
\hline -Open Wound - Head, Neck, Trunk & $870-879$ & 0 & 0 & 0 & 0 & 3 & 2 \\
\hline -Open Wound - Upper Limb & 880-887 & 0 & 0 & 0 & 0 & 0 & 0 \\
\hline -Superficial Injury & $910-919$ & 0 & 0 & 0 & 0 & 0 & 0 \\
\hline -Contusion & $920-924$ & 0 & 0 & 16 & 0 & 79 & 13 \\
\hline -Foreign Body Entering Orifice & $930-939$ & 0 & 0 & 0 & 0 & 0 & 0 \\
\hline $\begin{array}{l}\text {-Complications \& Unspecified } \\
\text { Injuries }\end{array}$ & 958-959 & 0 & 0 & 0 & 0 & 3 & 2 \\
\hline
\end{tabular}

\begin{tabular}{|c|c|c|c|c|c|c|c|c|c|}
\hline & & \multicolumn{8}{|c|}{ Men } \\
\hline & & \multicolumn{8}{|c|}{ Age Group } \\
\hline & & \multicolumn{2}{|c|}{$16-29$} & \multicolumn{2}{|c|}{$30-39$} & \multicolumn{2}{|c|}{40 - 49} & \multicolumn{2}{|c|}{$\mathbf{5 0}+$} \\
\hline & & $\begin{array}{c}\text { Days } \\
\text { Restricted }\end{array}$ & $\begin{array}{l}\text { Days } \\
\text { Lost }\end{array}$ & $\begin{array}{c}\text { Days } \\
\text { Restricted }\end{array}$ & $\begin{array}{l}\text { Days } \\
\text { Lost }\end{array}$ & $\begin{array}{c}\text { Days } \\
\text { Restricted }\end{array}$ & $\begin{array}{l}\text { Days } \\
\text { Lost }\end{array}$ & $\begin{array}{c}\text { Days } \\
\text { Restricted }\end{array}$ & $\begin{array}{l}\text { Days } \\
\text { Lost }\end{array}$ \\
\hline Diagnostic Category & ICD-9-CM Codes & & & & & & & & \\
\hline -Disorders of Eye & $360-379$ & 0 & 0 & 0 & 0 & 0 & 0 & 0 & 0 \\
\hline $\begin{array}{l}\text {-Other Dis Upper Respiratory } \\
\text { Tract }\end{array}$ & $470-478$ & 0 & 0 & 0 & 0 & 0 & 0 & 0 & 0 \\
\hline -Lung Dis from External Agents & $500-508$ & 0 & 0 & 0 & 0 & 0 & 0 & 0 & 0 \\
\hline -Arthropathies & $710-719$ & 0 & 0 & 0 & 0 & 54 & 0 & 47 & 0 \\
\hline -Dorsopathies & $720-724$ & 1 & 0 & 0 & 0 & 45 & 141 & 0 & 0 \\
\hline -Rheumatism, Excluding Back & $725-729$ & 0 & 0 & 0 & 0 & 0 & 0 & 47 & 0 \\
\hline -Symptoms & $780-789$ & 0 & 0 & 0 & 0 & 0 & 0 & 2 & 1 \\
\hline -Fracture - Neck, Trunk & $805-809$ & 0 & 0 & 0 & 0 & 0 & 0 & 1 & 10 \\
\hline -Fracture - Upper Limb & 810-819 & 0 & 0 & 0 & 0 & 0 & 0 & 41 & 31 \\
\hline -Fracture - Lower Limb & $820-829$ & 0 & 0 & 0 & 0 & 0 & 0 & 89 & 0 \\
\hline -Dislocation & 830-839 & 0 & 0 & 0 & 0 & 0 & 0 & 148 & 33 \\
\hline -Sprains \& Strains - Back & $846-847$ & 1 & 0 & 0 & 0 & 164 & 141 & 0 & 0 \\
\hline -Sprains \& Strains - Other & $840-845,848$ & 1 & 0 & 133 & 58 & 145 & 16 & 197 & 33 \\
\hline -Intracranial Injury & $850-854$ & 0 & 0 & 3 & 16 & 0 & 0 & 0 & 0 \\
\hline
\end{tabular}

(Continued)

*OSHA events with >1 ICD-9-CM code in the same diagnostic category were counted only once. Only those diagnostic categories and gender/age combinations with at least one occurrence appear in this table. 
Y-12 National Security Complex 2010

OSHA Data

Appendix P. Number of Workdays Lost or with Restricted Activity in Each Diagnostic Category by Gender and Age*

\begin{tabular}{|c|c|c|c|c|c|c|c|c|c|}
\hline & & \multicolumn{8}{|c|}{ Men } \\
\hline & & \multicolumn{8}{|c|}{ Age Group } \\
\hline & & \multicolumn{2}{|c|}{$16-29$} & \multicolumn{2}{|c|}{30 - 39} & \multicolumn{2}{|c|}{$40-49$} & \multicolumn{2}{|c|}{$50+$} \\
\hline & & $\begin{array}{c}\text { Days } \\
\text { Restricted }\end{array}$ & $\begin{array}{l}\text { Days } \\
\text { Lost }\end{array}$ & $\begin{array}{c}\text { Days } \\
\text { Restricted }\end{array}$ & $\begin{array}{l}\text { Days } \\
\text { Lost }\end{array}$ & $\begin{array}{c}\text { Days } \\
\text { Restricted }\end{array}$ & $\begin{array}{l}\text { Days } \\
\text { Lost }\end{array}$ & $\begin{array}{c}\text { Days } \\
\text { Restricted }\end{array}$ & $\begin{array}{l}\text { Days } \\
\text { Lost }\end{array}$ \\
\hline Diagnostic Category & ICD-9-CM Codes & & & & & & & & \\
\hline -Open Wound - Head, Neck, Trunk & 870-879 & 0 & 0 & 0 & 0 & 0 & 0 & 0 & 0 \\
\hline -Open Wound - Upper Limb & $880-887$ & 13 & 0 & 0 & 0 & 0 & 0 & 0 & 0 \\
\hline -Open Wound - Lower Limb & $890-897$ & 6 & 0 & 0 & 0 & 0 & 0 & 0 & 1 \\
\hline -Superficial Injury & $910-919$ & 0 & 0 & 0 & 0 & 0 & 0 & 1 & 11 \\
\hline -Contusion & $920-924$ & 6 & 0 & 0 & 0 & 125 & 0 & 1 & 11 \\
\hline -Crushing Injury & $925-929$ & 0 & 0 & 0 & 0 & 0 & 0 & 0 & 0 \\
\hline -Foreign Body Entering Orifice & 930-939 & 0 & 0 & 0 & 10 & 5 & 0 & 0 & 0 \\
\hline -Burns & 940-949 & 0 & 0 & 0 & 0 & 0 & 0 & 0 & 0 \\
\hline -Toxic Effects - Non-medicinal & 980-989 & 0 & 0 & 0 & 0 & 0 & 0 & 0 & 0 \\
\hline $\begin{array}{l}\text {-Unspecified Effects - External } \\
\text { Causes }\end{array}$ & $990-995$ & 0 & 0 & 0 & 0 & 0 & 0 & 0 & 0 \\
\hline
\end{tabular}

*OSHA events with >1 ICD-9-CM code in the same diagnostic category were counted only once. Only those diagnostic categories and gender/age combinations with at least one occurrence appear in this table. 
Y-12 National Security Complex 2010

OSHA Data

Appendix Q. Number of Occurrences in Each Accident Category by Gender and Age*

\begin{tabular}{|c|c|c|c|c|c|c|c|c|c|c|c|}
\hline & & \multicolumn{4}{|c|}{ Women } & \multicolumn{5}{|c|}{ Men } & \multirow[b]{3}{*}{ TOTAL } \\
\hline & & \multicolumn{3}{|c|}{ Age Group } & \multirow[b]{2}{*}{ TOTAL } & \multicolumn{4}{|c|}{ Age Group } & \multirow[b]{2}{*}{ TOTAL } & \\
\hline & & 30 - 39 & $40-49$ & $\begin{array}{c}50 \\
+\end{array}$ & & $16-29$ & 30 - 39 & $40-49$ & $\begin{array}{c}50 \\
+\end{array}$ & & \\
\hline Type of Accident & E Codes & & & & & & & & & & \\
\hline Motor Vehicle Traffic & E810-E819 & 0 & 0 & 0 & 0 & 0 & 1 & 0 & 0 & 1 & 1 \\
\hline Other Road Vehicle & E826-E829 & 0 & 0 & 0 & 0 & 0 & 0 & 0 & 1 & 1 & 1 \\
\hline Accidental Poisoning - Non-medicinal & E860-E869 & 0 & 0 & 0 & 0 & 0 & 0 & 0 & 1 & 1 & 1 \\
\hline Falls & E880-E888 & 2 & 3 & 7 & 12 & 0 & 0 & 2 & 4 & 6 & 18 \\
\hline Natural/Environmental Factors & E900-E909 & 0 & 0 & 0 & 0 & 1 & 0 & 1 & 2 & 4 & 4 \\
\hline $\begin{array}{l}\text { Submersion/Suffocation/Foreign } \\
\text { Bodies }\end{array}$ & E910-E915 & 0 & 1 & 0 & 1 & 1 & 0 & 1 & 1 & 3 & 4 \\
\hline Other Accidents & E916-E928 & 1 & 1 & 1 & 3 & 4 & 5 & 6 & 14 & 29 & 32 \\
\hline
\end{tabular}

*Only those accident types and gender/age combinations with at least one occurrence appear in this table. 
OSHA Data

Appendix R. Number of Workdays Lost or with Restricted Activity in Each Accident Category by Gender and Age*

\begin{tabular}{|c|c|c|c|c|c|c|c|}
\hline & & \multicolumn{6}{|c|}{ Women } \\
\hline & & \multicolumn{6}{|c|}{ Age Group } \\
\hline & & \multicolumn{2}{|c|}{30 - 39} & \multicolumn{2}{|c|}{$40-49$} & \multicolumn{2}{|c|}{$50+$} \\
\hline & & $\begin{array}{c}\text { Days } \\
\text { Restricted }\end{array}$ & Days Lost & $\begin{array}{c}\text { Days } \\
\text { Restricted }\end{array}$ & Days Lost & $\begin{array}{c}\text { Days } \\
\text { Restricted }\end{array}$ & Days Lost \\
\hline Type of Accident & E Codes & \multirow[b]{2}{*}{0} & \multirow[b]{2}{*}{0} & \multirow[b]{2}{*}{0} & \multirow[b]{2}{*}{0} & \multirow[b]{2}{*}{79} & \multirow[b]{2}{*}{170} \\
\hline Falls & E880-E888 & & & & & & \\
\hline $\begin{array}{l}\text { Submersion/Suffocation/Foreign } \\
\text { Bodies }\end{array}$ & E910-E915 & 0 & 0 & 0 & 0 & 0 & 0 \\
\hline Other Accidents & E916-E928 & 0 & 0 & 16 & 0 & 0 & 0 \\
\hline
\end{tabular}

\begin{tabular}{|c|c|c|c|c|c|c|c|c|c|}
\hline & & \multicolumn{8}{|c|}{ Men } \\
\hline & & \multicolumn{8}{|c|}{ Age Group } \\
\hline & & \multicolumn{2}{|c|}{$16-29$} & \multicolumn{2}{|c|}{$30-39$} & \multicolumn{2}{|c|}{$40-49$} & \multicolumn{2}{|c|}{$50+$} \\
\hline & & $\begin{array}{c}\text { Days } \\
\text { Restricted }\end{array}$ & $\begin{array}{l}\text { Days } \\
\text { Lost }\end{array}$ & $\begin{array}{c}\text { Days } \\
\text { Restricted }\end{array}$ & $\begin{array}{l}\text { Days } \\
\text { Lost }\end{array}$ & $\begin{array}{c}\text { Days } \\
\text { Restricted }\end{array}$ & $\begin{array}{l}\text { Days } \\
\text { Lost }\end{array}$ & $\begin{array}{c}\text { Days } \\
\text { Restricted }\end{array}$ & $\begin{array}{l}\text { Days } \\
\text { Lost }\end{array}$ \\
\hline Type of Accident & E Codes & \multirow[b]{2}{*}{0} & \multirow[b]{2}{*}{0} & \multirow[b]{2}{*}{0} & \multirow[b]{2}{*}{5} & \multirow[b]{2}{*}{0} & \multirow[b]{2}{*}{0} & \multirow[b]{2}{*}{0} & \multirow[b]{2}{*}{0} \\
\hline Motor Vehicle Traffic & E810-E819 & & & & & & & & \\
\hline Other Road Vehicle & E826-E829 & 0 & 0 & 0 & 0 & 0 & 0 & 0 & 1 \\
\hline Accidental Poisoning - Non-medicinal & E860-E869 & 0 & 0 & 0 & 0 & 0 & 0 & 0 & 0 \\
\hline Falls & E880-E888 & 0 & 0 & 0 & 0 & 216 & 16 & 149 & 60 \\
\hline Natural/Environmental Factors & E900-E909 & 0 & 0 & 0 & 0 & 0 & 0 & 0 & 0 \\
\hline $\begin{array}{l}\text { Submersion/Suffocation/Foreign } \\
\text { Bodies }\end{array}$ & E910-E915 & 0 & 0 & 0 & 0 & 5 & 0 & 0 & 0 \\
\hline Other Accidents & E916-E928 & 20 & 0 & 136 & 79 & 99 & 141 & 179 & 14 \\
\hline
\end{tabular}

*OSHA events with $>1 \mathrm{E}$ code in the same accident type were counted only once. Only those accident types and gender/age combinations with at least one occurrence appear in this table. 


\section{Y-12 National Security Complex 2010}

OSHA Data

Appendix S. Number of Diagnoses in Each Diagnostic Category by Gender and Job Category*

\begin{tabular}{|c|c|c|c|c|c|}
\hline & & \multicolumn{4}{|c|}{ Women } \\
\hline & & \multicolumn{3}{|c|}{ Job Category } & \multirow[b]{2}{*}{ TOTAL } \\
\hline & & Professional & Service & Crafts & \\
\hline Diagnostic Category & ICD-9-CM Code & & & & \\
\hline $\begin{array}{l}\text { MUSCULOSKELETAL } \\
\text { \& CONNECTIVE } \\
\text { TISSUE }\end{array}$ & $710-739$ & 0 & 0 & 1 & 1 \\
\hline -Arthropathies & $710-719$ & 0 & 0 & 1 & 1 \\
\hline $\begin{array}{l}\text { SYMPTOMS, } \\
\text { SIGNS, \& ILL-DEFINED } \\
\text { CONDITIONS }\end{array}$ & 780-799 & 0 & 0 & 1 & 1 \\
\hline -Symptoms & 780-789 & 0 & 0 & 1 & 1 \\
\hline INJURY \& POISONING & $800-999$ & 2 & 9 & 19 & 30 \\
\hline -Fracture - Upper Limb & $810-819$ & 0 & 0 & 1 & 1 \\
\hline -Dislocation & $830-839$ & 0 & 0 & 1 & 1 \\
\hline -Sprains \& Strains - Back & \begin{tabular}{|l|}
$846-847$ \\
\end{tabular} & 0 & 0 & 1 & 1 \\
\hline $\begin{array}{l}\text {-Sprains \& Strains - } \\
\text { Other }\end{array}$ & $840-845,848$ & 0 & 2 & 3 & 5 \\
\hline -Intracranial Injury & $850-854$ & 1 & 0 & 0 & 1 \\
\hline $\begin{array}{l}\text {-Open Wound - Head, } \\
\text { Neck, Trunk }\end{array}$ & $870-879$ & 0 & 0 & 2 & 2 \\
\hline $\begin{array}{l}\text {-Open Wound - Upper } \\
\text { Limb }\end{array}$ & $880-887$ & 0 & 1 & 1 & 2 \\
\hline -Superficial Injury & 910-919 & 0 & 2 & 0 & 2 \\
\hline -Contusion & $920-924$ & 1 & 3 & 9 & 13 \\
\hline $\begin{array}{l}\text {-Foreign Body Entering } \\
\text { Orifice }\end{array}$ & \begin{tabular}{|l|}
$930-939$ \\
\end{tabular} & 0 & 1 & 0 & 1 \\
\hline $\begin{array}{l}\text {-Complications \& } \\
\text { Unspecified Injuries }\end{array}$ & \begin{tabular}{|l|}
$958-959$ \\
\end{tabular} & 0 & 0 & 1 & 1 \\
\hline
\end{tabular}

\begin{tabular}{|c|c|c|c|c|}
\hline & \multicolumn{4}{|c|}{ Women } \\
\hline & \multicolumn{3}{|c|}{ Job Category } & \multirow[b]{2}{*}{ TOTAL } \\
\hline & Professional & Service & Crafts & \\
\hline Diagnostic Category & \multirow[b]{2}{*}{2} & \multirow[b]{2}{*}{9} & \multirow[b]{2}{*}{21} & \multirow[b]{2}{*}{32} \\
\hline Total & & & & \\
\hline
\end{tabular}

*Only those diagnostic categories and gender/job category combinations with at least one occurrence appear in this table. 


\section{Y-12 National Security Complex 2010}

OSHA Data

Appendix S. Number of Diagnoses in Each Diagnostic Category by Gender and Job Category*

\begin{tabular}{|c|c|c|c|c|c|}
\hline & & \multicolumn{4}{|c|}{ Men } \\
\hline & & \multicolumn{3}{|c|}{ Job Category } & \multirow[b]{2}{*}{ TOTAL } \\
\hline & & Professional & Service & Crafts & \\
\hline Diagnostic Category & ICD-9-CM Code & & & & \\
\hline $\begin{array}{l}\text { NERVOUS SYSTEM } \\
\text { (NS) \& SENSE ORGANS }\end{array}$ & $320-389$ & 0 & 0 & 2 & 2 \\
\hline -Disorders of Eye & $360-379$ & 0 & 0 & 2 & 2 \\
\hline $\begin{array}{l}\text { RESPIRATORY } \\
\text { SYSTEM }\end{array}$ & $460-519$ & 0 & 0 & 4 & 4 \\
\hline $\begin{array}{l}\text {-Other Dis Upper } \\
\text { Respiratory Tract }\end{array}$ & $470-478$ & 0 & 0 & 1 & 1 \\
\hline $\begin{array}{l}\text {-Lung Dis from External } \\
\text { Agents }\end{array}$ & $500-508$ & 0 & 0 & 3 & 3 \\
\hline $\begin{array}{l}\text { MUSCULOSKELETAL } \\
\text { \& CONNECTIVE } \\
\text { TISSUE }\end{array}$ & $710-739$ & 0 & 4 & 12 & 16 \\
\hline -Arthropathies & $710-719$ & 0 & 0 & 5 & 5 \\
\hline -Dorsopathies & $720-724$ & 0 & 4 & 5 & 9 \\
\hline $\begin{array}{l}\text {-Rheumatism, Excluding } \\
\text { Back }\end{array}$ & $725-729$ & 0 & 0 & 2 & 2 \\
\hline $\begin{array}{l}\text { SYMPTOMS, } \\
\text { SIGNS, \& ILL-DEFINED } \\
\text { CONDITIONS }\end{array}$ & $780-799$ & 0 & 1 & 3 & 4 \\
\hline -Symptoms & 780-789 & 0 & 1 & 3 & 4 \\
\hline INJURY \& POISONING & 800-999 & 2 & 18 & 38 & 58 \\
\hline -Fracture - Neck, Trunk & 805-809 & 0 & 0 & 1 & 1 \\
\hline -Fracture - Upper Limb & 810-819 & 0 & 1 & 2 & 3 \\
\hline -Fracture - Lower Limb & $820-829$ & 0 & 0 & 1 & 1 \\
\hline -Dislocation & 830-839 & 0 & 1 & 1 & 2 \\
\hline -Sprains \& Strains - Back & 846-847 & 0 & 2 & 4 & 6 \\
\hline $\begin{array}{l}\text {-Sprains \& Strains - } \\
\text { Other }\end{array}$ & $840-845,848$ & 0 & 4 & 6 & 10 \\
\hline -Intracranial Injury & $850-854$ & 0 & 1 & 1 & 2 \\
\hline $\begin{array}{l}\text {-Open Wound - Head, } \\
\text { Neck, Trunk }\end{array}$ & $870-879$ & 0 & 1 & 0 & 1 \\
\hline $\begin{array}{l}\text {-Open Wound - Upper } \\
\text { Limb }\end{array}$ & $880-887$ & 0 & 1 & 4 & 5 \\
\hline $\begin{array}{l}\text {-Open Wound - Lower } \\
\text { Limb }\end{array}$ & $890-897$ & 0 & 1 & 1 & 2 \\
\hline -Superficial Injury & 910-919 & 1 & 1 & 1 & 3 \\
\hline -Contusion & $920-924$ & 1 & 3 & 5 & 9 \\
\hline -Crushing Injury & 925-929 & 0 & 0 & 1 & 1 \\
\hline $\begin{array}{l}\text {-Foreign Body Entering } \\
\text { Orifice }\end{array}$ & $930-939$ & 0 & 0 & 5 & 5 \\
\hline -Burns & $940-949$ & 0 & 2 & 0 & 2 \\
\hline
\end{tabular}

(Continued)

*Only those diagnostic categories and gender/job category combinations with at least one occurrence appear in this table. 


\section{Y-12 National Security Complex 2010}

OSHA Data

Appendix S. Number of Diagnoses in Each Diagnostic Category by Gender and Job Category*

\begin{tabular}{|c|c|c|c|c|c|}
\hline & & \multicolumn{4}{|c|}{ Men } \\
\hline & & \multicolumn{3}{|c|}{ Job Category } & \multirow[b]{2}{*}{ TOTAL } \\
\hline & & Professional & Service & Crafts & \\
\hline Diagnostic Category & ICD-9-CM Code & & & & \\
\hline $\begin{array}{l}\text {-Toxic } \\
\text { Effects - Non-medicinal }\end{array}$ & \begin{tabular}{|c|}
$980-989$ \\
\end{tabular} & 0 & 0 & 4 & 4 \\
\hline $\begin{array}{l}\text {-Unspecified } \\
\text { Effects - External Causes }\end{array}$ & $990-995$ & 0 & 0 & 1 & 1 \\
\hline
\end{tabular}

\begin{tabular}{|c|c|c|c|c|}
\hline & \multicolumn{4}{|c|}{ Men } \\
\hline & \multicolumn{3}{|c|}{ Job Category } & \multirow[b]{2}{*}{ TOTAL } \\
\hline & Professional & Service & Crafts & \\
\hline Diagnostic Category & \multirow[b]{2}{*}{2} & \multirow[b]{2}{*}{23} & \multirow[b]{2}{*}{59} & \multirow[b]{2}{*}{84} \\
\hline Total & & & & \\
\hline
\end{tabular}

*Only those diagnostic categories and gender/job category combinations with at least one occurrence appear in this table. 


\section{Y-12 National Security Complex 2010}

OSHA Data

Appendix T. Number of Workdays Lost or with Restricted Activity in Each Diagnostic Category by Gender and Job Category*

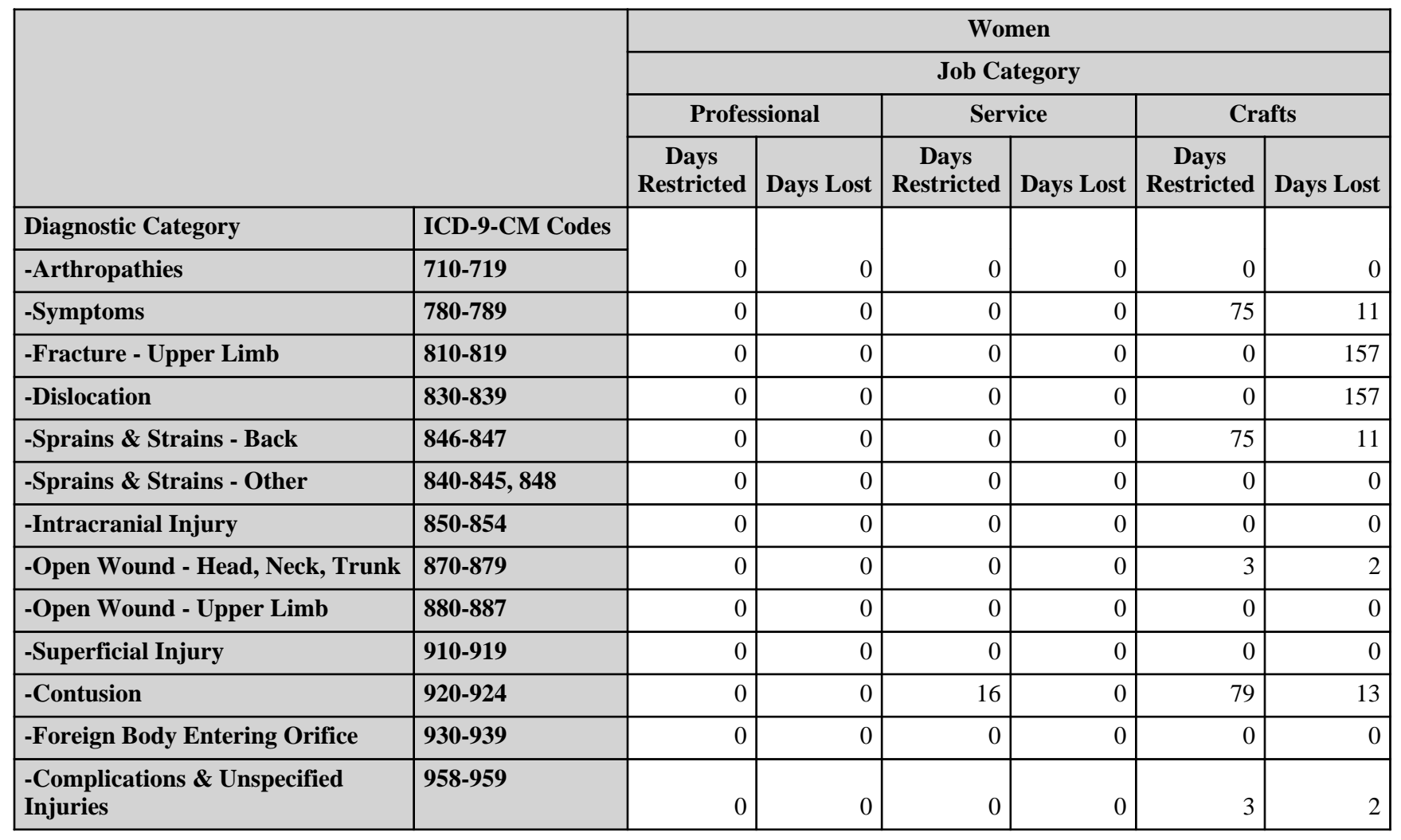

\begin{tabular}{|c|c|c|c|c|c|c|c|}
\hline & & \multicolumn{6}{|c|}{ Men } \\
\hline & & \multicolumn{6}{|c|}{ Job Category } \\
\hline & & \multicolumn{2}{|c|}{ Professional } & \multicolumn{2}{|c|}{ Service } & \multicolumn{2}{|c|}{ Crafts } \\
\hline & & $\begin{array}{c}\text { Days } \\
\text { Restricted }\end{array}$ & Days Lost & \begin{tabular}{|c|} 
Days \\
Restricted
\end{tabular} & Days Lost & $\begin{array}{c}\text { Days } \\
\text { Restricted }\end{array}$ & Days Lost \\
\hline Diagnostic Category & ICD-9-CM Codes & \multirow[b]{2}{*}{0} & \multirow[b]{2}{*}{0} & \multirow[b]{2}{*}{0} & \multirow[b]{2}{*}{0} & \multirow[b]{2}{*}{0} & \multirow[b]{2}{*}{0} \\
\hline -Disorders of Eye & $360-379$ & & & & & & \\
\hline $\begin{array}{l}\text {-Other Dis Upper Respiratory } \\
\text { Tract }\end{array}$ & $470-478$ & 0 & 0 & 0 & 0 & 0 & 0 \\
\hline -Lung Dis from External Agents & $500-508$ & 0 & 0 & 0 & 0 & 0 & 0 \\
\hline -Arthropathies & $710-719$ & 0 & 0 & 0 & 0 & 101 & 0 \\
\hline -Dorsopathies & $720-724$ & 0 & 0 & 1 & 0 & 45 & 141 \\
\hline -Rheumatism, Excluding Back & 725-729 & 0 & 0 & 0 & 0 & 47 & 0 \\
\hline -Symptoms & $780-789$ & 0 & 0 & 2 & 1 & 0 & 0 \\
\hline -Fracture - Neck, Trunk & $805-809$ & 0 & 0 & 0 & 0 & 1 & 10 \\
\hline -Fracture - Upper Limb & $810-819$ & 0 & 0 & 41 & 13 & 0 & 18 \\
\hline -Fracture - Lower Limb & $820-829$ & 0 & 0 & 0 & 0 & 89 & 0 \\
\hline -Dislocation & 830-839 & 0 & 0 & 0 & 1 & 148 & 32 \\
\hline -Sprains \& Strains - Back & 846-847 & 0 & 0 & 1 & 0 & 164 & 141 \\
\hline -Sprains \& Strains - Other & $840-845,848$ & 0 & 0 & 95 & 43 & 381 & 64 \\
\hline -Intracranial Injury & $850-854$ & 0 & 0 & 0 & 5 & 3 & 11 \\
\hline
\end{tabular}

(Continued)

*OSHA events with >1 ICD-9-CM code in the same diagnostic category were counted only once. Only those diagnostic categories and gender/job category combinations with at least one occurrence appear in this table. 
Y-12 National Security Complex 2010

OSHA Data

Appendix T. Number of Workdays Lost or with Restricted Activity in Each Diagnostic Category by Gender and Job Category*

\begin{tabular}{|c|c|c|c|c|c|c|c|}
\hline & & \multicolumn{6}{|c|}{ Men } \\
\hline & & \multicolumn{6}{|c|}{ Job Category } \\
\hline & & \multicolumn{2}{|c|}{ Professional } & \multicolumn{2}{|c|}{ Service } & \multicolumn{2}{|c|}{ Crafts } \\
\hline & & $\begin{array}{c}\text { Days } \\
\text { Restricted }\end{array}$ & Days Lost & \begin{tabular}{|c} 
Days \\
Restricted
\end{tabular} & Days Lost & \begin{tabular}{|c|} 
Days \\
Restricted
\end{tabular} & Days Lost \\
\hline Diagnostic Category & ICD-9-CM Codes & & & & & & \\
\hline -Open Wound - Head, Neck, Trunk & $870-879$ & 0 & 0 & 0 & 0 & 0 & 0 \\
\hline -Open Wound - Upper Limb & $880-887$ & 0 & 0 & 0 & 0 & 13 & 0 \\
\hline -Open Wound - Lower Limb & $890-897$ & 0 & 0 & 0 & 1 & 6 & 0 \\
\hline -Superficial Injury & 910-919 & 0 & 0 & 0 & 1 & 1 & 10 \\
\hline -Contusion & 920-924 & 0 & 0 & 0 & 1 & 132 & 10 \\
\hline -Crushing Injury & 925-929 & 0 & 0 & 0 & 0 & 0 & 0 \\
\hline -Foreign Body Entering Orifice & 930-939 & 0 & 0 & 0 & 0 & 5 & 10 \\
\hline -Burns & 940-949 & 0 & 0 & 0 & 0 & 0 & 0 \\
\hline -Toxic Effects - Non-medicinal & 980-989 & 0 & 0 & 0 & 0 & 0 & 0 \\
\hline $\begin{array}{l}\text {-Unspecified Effects - External } \\
\text { Causes }\end{array}$ & $990-995$ & 0 & 0 & 0 & 0 & 0 & 0 \\
\hline
\end{tabular}

*OSHA events with >1 ICD-9-CM code in the same diagnostic category were counted only once. Only those diagnostic categories and gender/job category combinations with at least one occurrence appear in this table. 
Y-12 National Security Complex 2010

OSHA Data

Appendix U. Number of Occurrences in Each Accident Category by Gender and Job Category*

\begin{tabular}{|c|c|c|c|c|c|}
\hline & & \multicolumn{4}{|c|}{ Women } \\
\hline & & \multicolumn{3}{|c|}{ Job Category } & \multirow[b]{2}{*}{ TOTAL } \\
\hline & & Professional & Service & Crafts & \\
\hline Type of Accident & E CODES & \multirow[b]{2}{*}{1} & \multirow[b]{2}{*}{3} & \multirow[b]{2}{*}{8} & \multirow[b]{2}{*}{12} \\
\hline Falls & E880-E888 & & & & \\
\hline $\begin{array}{l}\text { Submersion/Suffocation/Foreign } \\
\text { Bodies }\end{array}$ & E910-E915 & 0 & 1 & 0 & 1 \\
\hline Other Accidents & E916-E928 & 0 & 2 & 1 & 3 \\
\hline
\end{tabular}

\begin{tabular}{|c|c|c|c|c|c|}
\hline & & \multicolumn{4}{|c|}{ Men } \\
\hline & & \multicolumn{3}{|c|}{ Job Category } & \multirow[b]{2}{*}{ TOTAL } \\
\hline & & Professional & Service & Crafts & \\
\hline Type of Accident & E CODES & \multirow[b]{2}{*}{0} & \multirow[b]{2}{*}{1} & \multirow[b]{2}{*}{0} & \multirow[b]{2}{*}{1} \\
\hline Motor Vehicle Traffic & E810-E819 & & & & \\
\hline Other Road Vehicle & E826-E829 & 0 & 1 & 0 & 1 \\
\hline Accidental Poisoning - Non-medicinal & E860-E869 & 0 & 0 & 1 & 1 \\
\hline Falls & E880-E888 & 0 & 2 & 4 & 6 \\
\hline Natural/Environmental Factors & E900-E909 & 0 & 0 & 4 & 4 \\
\hline $\begin{array}{l}\text { Submersion/Suffocation/Foreign } \\
\text { Bodies }\end{array}$ & E910-E915 & 0 & 0 & 3 & 3 \\
\hline Other Accidents & E916-E928 & 1 & 9 & 19 & 29 \\
\hline
\end{tabular}

*Only those accident types and gender/job category combinations with at least one occurrence appear in this table. 


\section{Y-12 National Security Complex 2010}

OSHA Data

Appendix V. Number of Workdays Lost or with Restricted Activity in Each Accident Category by Gender and Job Category*

\begin{tabular}{|c|c|c|c|c|c|c|c|}
\hline & & \multicolumn{6}{|c|}{ Women } \\
\hline & & \multicolumn{6}{|c|}{ Job Category } \\
\hline & & \multicolumn{2}{|c|}{ Professional } & \multicolumn{2}{|c|}{ Service } & \multicolumn{2}{|c|}{ Crafts } \\
\hline & & $\begin{array}{c}\text { Days } \\
\text { Restricted }\end{array}$ & Days Lost & $\begin{array}{c}\text { Days } \\
\text { Restricted }\end{array}$ & Days Lost & $\begin{array}{c}\text { Days } \\
\text { Restricted }\end{array}$ & Days Lost \\
\hline Type of Accident & E Codes & \multirow[b]{2}{*}{0} & \multirow[b]{2}{*}{0} & \multirow[b]{2}{*}{0} & \multirow[b]{2}{*}{0} & \multirow[b]{2}{*}{79} & \multirow[b]{2}{*}{170} \\
\hline Falls & E880-E888 & & & & & & \\
\hline $\begin{array}{l}\text { Submersion/Suffocation/Foreign } \\
\text { Bodies }\end{array}$ & E910-E915 & 0 & 0 & 0 & 0 & 0 & 0 \\
\hline Other Accidents & \begin{tabular}{|l|} 
E916-E928 \\
\end{tabular} & 0 & 0 & 16 & 0 & 0 & 0 \\
\hline
\end{tabular}

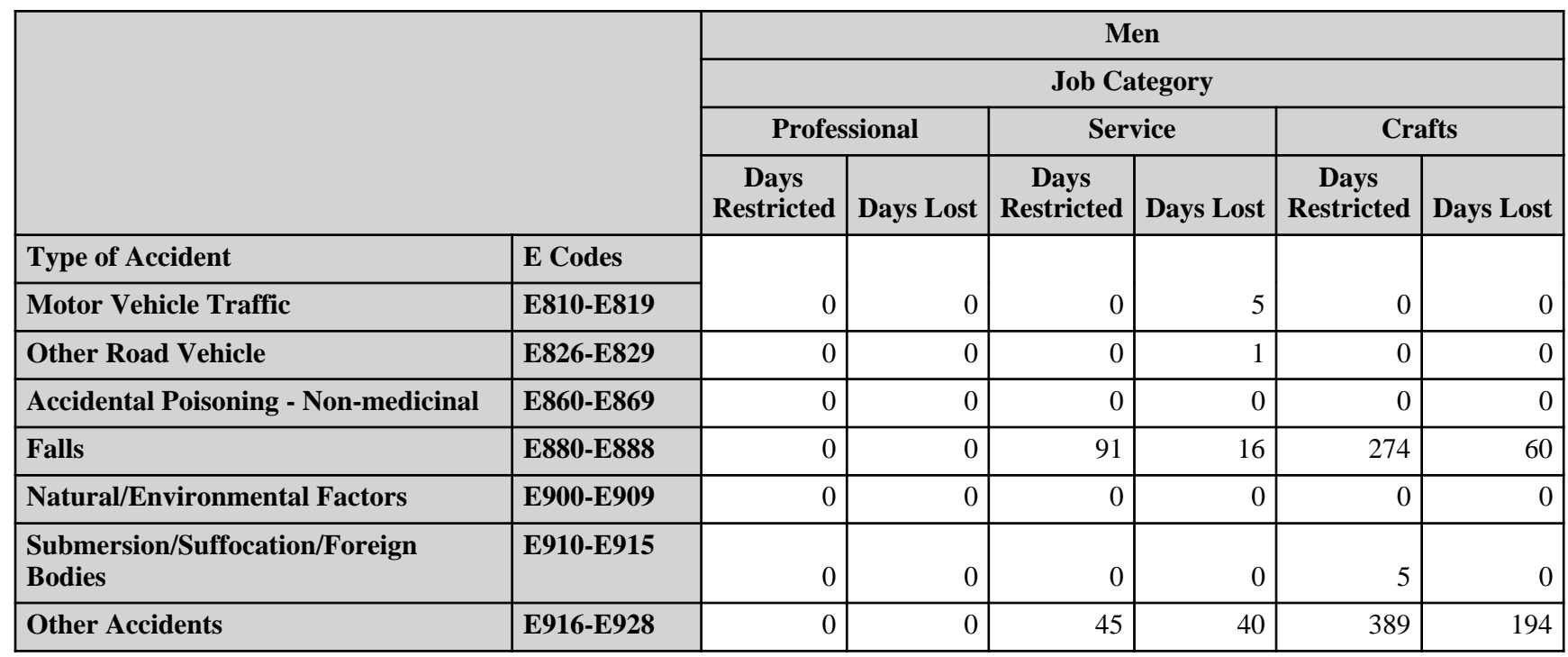

*OSHA events with >1 E code in the same accident type were counted only once. Only those accident types and gender/job category combinations with at least one occurrence appear in this table. 


\section{Y-12 National Security Complex 2010}

OSHA Data

Appendix W. Age-Adjusted OSHA Illness and Injury Rates by Diagnostic Category*

Part 1. Men

\begin{tabular}{|c|c|c|c|c|c|}
\hline & & $\begin{array}{l}\text { Number of } \\
\text { Diagnoses }\end{array}$ & $\begin{array}{l}\text { Age-Adjusted } \\
\text { Rate per } 1,000 * *\end{array}$ & $\begin{array}{c}\text { Lower } 95 \% \\
\text { Confidence } \\
\text { Limit per 1,000 }\end{array}$ & $\begin{array}{c}\text { Upper } 95 \% \\
\text { Confidence } \\
\text { Limit per 1,000 }\end{array}$ \\
\hline Diagnostic Category & ICD-9-CM Code & \multirow[b]{2}{*}{2} & \multirow[b]{2}{*}{0.2} & \multirow[b]{2}{*}{0.1} & \multirow[b]{2}{*}{1.0} \\
\hline NERVOUS SYSTEM (NS) \& SENSE ORGANS & 320-389 & & & & \\
\hline -Disorders of Eye & $360-379$ & 2 & 0.2 & 0.1 & 1.0 \\
\hline RESPIRATORY SYSTEM & $460-519$ & 4 & 0.7 & 0.2 & 2.0 \\
\hline -Other Dis Upper Respiratory Tract & $470-478$ & 1 & 0.1 & 0.0 & 0.9 \\
\hline -Lung Dis from External Agents & $500-508$ & 3 & 0.6 & 0.2 & 1.9 \\
\hline $\begin{array}{l}\text { MUSCULOSKELETAL \& CONNECTIVE } \\
\text { TISSUE }\end{array}$ & 710-739 & 16 & 5.2 & 2.8 & 9.5 \\
\hline -Arthropathies & $710-719$ & 5 & 0.9 & 0.4 & 2.2 \\
\hline -Dorsopathies & \begin{tabular}{|c|}
$720-724$ \\
\end{tabular} & 9 & 4.0 & 1.9 & 8.5 \\
\hline -Rheumatism, Excluding Back & 725-729 & 2 & 0.3 & 0.1 & 1.2 \\
\hline $\begin{array}{l}\text { SYMPTOMS, SIGNS, \& ILL-DEFINED } \\
\text { CONDITIONS }\end{array}$ & $780-799$ & 4 & 0.5 & 0.2 & 1.3 \\
\hline -Symptoms & 780-789 & 4 & 0.5 & 0.2 & 1.3 \\
\hline INJURY \& POISONING & $800-999$ & 58 & 16.5 & 11.9 & 22.9 \\
\hline -Fracture - Skull & $800-804$ & 1 & 0.1 & 0.0 & 0.9 \\
\hline -Fracture - Upper Limb & 810-819 & 3 & 0.5 & 0.2 & 1.5 \\
\hline -Fracture - Lower Limb & $820-829$ & 1 & 0.1 & 0.0 & 0.9 \\
\hline -Dislocation & $830-839$ & 2 & 0.2 & 0.1 & 1.0 \\
\hline -Sprains \& Strains - Back & 846-847 & 6 & 2.2 & 0.9 & 5.5 \\
\hline -Sprains \& Strains - Other & $840-845,848$ & 10 & 2.9 & 1.4 & 6.2 \\
\hline -Open Wound - Head, Neck, Trunk & $870-879$ & 1 & 0.2 & 0.0 & 1.3 \\
\hline -Open Wound - Upper Limb & 880-887 & 5 & 1.5 & 0.5 & 4.7 \\
\hline -Open Wound - Lower Limb & 890-897 & 2 & 0.9 & 0.2 & 5.0 \\
\hline -Superficial Injury & 910-919 & 3 & 0.4 & 0.1 & 1.1 \\
\hline -Contusion & $920-924$ & 9 & 2.6 & 1.0 & 6.3 \\
\hline -Foreign Body Entering Orifice & 930-939 & 5 & 1.7 & 0.6 & 5.1 \\
\hline -Burns & 940-949 & 2 & 0.6 & 0.1 & 2.2 \\
\hline -Toxic Effects - Non-medicinal & 980-989 & 5 & 1.5 & 0.5 & 4.7 \\
\hline -Complications of Surgical/Medical Care & 996-999 & 3 & 1.2 & 0.4 & 3.6 \\
\hline Total & & 84 & 23.1 & 17.6 & 30.4 \\
\hline
\end{tabular}

* Only those diagnostic categories with at least one occurrence appear in this table.

**Standardized to age distribution of 2000 U.S. population. 
Y-12 National Security Complex 2010

OSHA Data

Appendix W. Age-Adjusted OSHA Illness and Injury Rates by Diagnostic Category*

Part 2. Women

\begin{tabular}{|c|c|c|c|c|c|}
\hline & & $\begin{array}{l}\text { Number of } \\
\text { Diagnoses }\end{array}$ & $\begin{array}{l}\text { Age-Adjusted } \\
\text { Rate per } 1,000 * *\end{array}$ & $\begin{array}{c}\text { Lower } 95 \% \\
\text { Confidence } \\
\text { Limit per } 1,000\end{array}$ & $\begin{array}{c}\text { Upper } 95 \% \\
\text { Confidence } \\
\text { Limit per 1,000 }\end{array}$ \\
\hline Diagnostic Category & ICD-9-CM Code & & & & \\
\hline $\begin{array}{l}\text { MUSCULOSKELETAL \& CONNECTIVE } \\
\text { TISSUE }\end{array}$ & 710-739 & 1 & 0.9 & 0.1 & 6.4 \\
\hline -Arthropathies & $710-719$ & 1 & 0.9 & 0.1 & 6.4 \\
\hline $\begin{array}{l}\text { SYMPTOMS, SIGNS, \& ILL-DEFINED } \\
\text { CONDITIONS }\end{array}$ & 780-799 & 1 & 0.3 & 0.0 & 2.2 \\
\hline -Symptoms & 780-789 & 1 & 0.3 & 0.0 & 2.2 \\
\hline INJURY \& POISONING & $800-999$ & 30 & 18.5 & 12.2 & 28.1 \\
\hline -Fracture - Upper Limb & 810-819 & 1 & 0.9 & 0.1 & 6.4 \\
\hline -Dislocation & $830-839$ & 1 & 0.9 & 0.1 & 6.4 \\
\hline -Sprains \& Strains - Back & 846-847 & 1 & 0.3 & 0.0 & 2.2 \\
\hline -Sprains \& Strains - Other & $840-845,848$ & 5 & 3.7 & 1.4 & 10.0 \\
\hline -Open Wound - Head, Neck, Trunk & 870-879 & 2 & 0.6 & 0.2 & 2.5 \\
\hline -Open Wound - Upper Limb & 880-887 & 2 & 1.7 & 0.3 & 8.9 \\
\hline -Superficial Injury & 910-919 & 2 & 1.7 & 0.3 & 8.9 \\
\hline -Contusion & $920-924$ & 13 & 7.1 & 3.8 & 13.3 \\
\hline -Foreign Body Entering Orifice & 930-939 & 1 & 0.6 & 0.1 & 4.0 \\
\hline -Complications of Surgical/Medical Care & 996-999 & 2 & 0.9 & 0.2 & 3.7 \\
\hline Total & & 32 & 19.7 & 13.1 & 29.5 \\
\hline
\end{tabular}

* Only those diagnostic categories with at least one occurrence appear in this table.

**Standardized to age distribution of 2000 U.S. population. 


\section{Y-12 National Security Complex 2010}

OSHA Data

Appendix W. Age-Adjusted OSHA Illness and Injury Rates by Diagnostic Category*

Part 3. Men and Women

\begin{tabular}{|c|c|c|c|c|c|}
\hline & & $\begin{array}{c}\text { Number of } \\
\text { Diagnoses }\end{array}$ & $\begin{array}{c}\text { Age-Adjusted } \\
\text { Rate per } 1,000 * *\end{array}$ & $\begin{array}{c}\text { Lower } 95 \% \\
\text { Confidence } \\
\text { Limit per 1,000 }\end{array}$ & $\begin{array}{c}\text { Upper } 95 \% \\
\text { Confidence } \\
\text { Limit per 1,000 }\end{array}$ \\
\hline Diagnostic Category & ICD-9-CM Code & & & & \\
\hline NERVOUS SYSTEM (NS) \& SENSE ORGANS & 320-389 & 2 & 0.2 & 0.0 & 0.7 \\
\hline -Disorders of Eye & $360-379$ & 2 & 0.2 & 0.0 & 0.7 \\
\hline RESPIRATORY SYSTEM & $460-519$ & 4 & 0.5 & 0.2 & 1.5 \\
\hline -Other Dis Upper Respiratory Tract & $470-478$ & 1 & 0.1 & 0.0 & 0.6 \\
\hline -Lung Dis from External Agents & $500-508$ & 3 & 0.4 & 0.1 & 1.4 \\
\hline $\begin{array}{l}\text { MUSCULOSKELETAL \& CONNECTIVE } \\
\text { TISSUE }\end{array}$ & 710-739 & 17 & 4.0 & 2.2 & 7.1 \\
\hline -Arthropathies & 710-719 & 6 & 0.8 & 0.4 & 1.9 \\
\hline -Dorsopathies & $720-724$ & 9 & 2.9 & 1.4 & 6.2 \\
\hline -Rheumatism, Excluding Back & 725-729 & 2 & 0.2 & 0.1 & 1.0 \\
\hline $\begin{array}{l}\text { SYMPTOMS, SIGNS, \& ILL-DEFINED } \\
\text { CONDITIONS }\end{array}$ & $780-799$ & 5 & 0.4 & 0.2 & 1.1 \\
\hline -Symptoms & 780-789 & 5 & 0.4 & 0.2 & 1.1 \\
\hline INJURY \& POISONING & 800-999 & 88 & 16.8 & 12.9 & 21.8 \\
\hline -Fracture - Skull & $800-804$ & 1 & 0.1 & 0.0 & 0.6 \\
\hline -Fracture - Upper Limb & 810-819 & 4 & 0.6 & 0.2 & 1.5 \\
\hline -Fracture - Lower Limb & $820-829$ & 1 & 0.1 & 0.0 & 0.6 \\
\hline -Dislocation & 830-839 & 3 & 0.3 & 0.1 & 1.1 \\
\hline -Sprains \& Strains - Back & 846-847 & 7 & 1.6 & 0.7 & 4.0 \\
\hline -Sprains \& Strains - Other & $840-845,848$ & 15 & 3.1 & 1.7 & 5.7 \\
\hline -Open Wound - Head, Neck, Trunk & 870-879 & 3 & 0.3 & 0.1 & 1.1 \\
\hline -Open Wound - Upper Limb & 880-887 & 7 & 1.5 & 0.6 & 3.9 \\
\hline -Open Wound - Lower Limb & 890-897 & 2 & 0.7 & 0.1 & 3.7 \\
\hline -Superficial Injury & 910-919 & 5 & 0.7 & 0.2 & 2.0 \\
\hline -Contusion & $920-924$ & 22 & 3.7 & 2.1 & 6.4 \\
\hline -Foreign Body Entering Orifice & 930-939 & 6 & 1.5 & 0.5 & 3.9 \\
\hline -Burns & 940-949 & 2 & 0.4 & 0.1 & 1.5 \\
\hline -Toxic Effects - Non-medicinal & 980-989 & 5 & 1.1 & 0.4 & 3.4 \\
\hline -Complications of Surgical/Medical Care & 996-999 & 5 & 1.1 & 0.4 & 2.9 \\
\hline Total & & 116 & 21.9 & 17.4 & 27.5 \\
\hline
\end{tabular}

* Only those diagnostic categories with at least one occurrence appear in this table.

**Standardized to age distribution of 2000 U.S. population. 